\title{
Polyhedra Dual to the Weyl Chamber Decomposition: A Précis ${ }^{\dagger}$
}

\author{
By
}

Kyoji SAITO*

\begin{abstract}
Let $V_{\mathbb{R}}$ be a real vector space with an irreducible action of a finite reflection group $W$. We study the semi-algebraic geometry of the $W$-quotient affine variety $V / / W$ with the discriminant divisor $D_{W}$ in it and the $\tau$-quotient affine variety $V / / W / / \tau$ with the bifurcation set $B_{W}$ in it, where $\tau$ is the $\mathbb{G}_{a}$-action on $V / / W$ obtained by the integration of the primitive vector field $D$ on $V / / W$ and $B_{W}$ is the discriminant divisor of the induced projection $: D_{W} \rightarrow V / / W / / \tau$.

Our goal is the construction of a one-parameter family of the semi-algebraic polyhedra $K_{W}(\lambda)$ in $V_{\mathbb{R}}$ which are dual to the Weyl chamber decomposition of $V_{\mathbb{R}}$.

As an application, we obtain two geometric descriptions of generators for $\pi_{1}\left((V / / W)_{\mathbb{C}}^{\text {reg }}\right)$, satisfying the Artin braid relations.

The key of the construction of the polyhedra $K_{W}(\lambda)$ is a theorem on a linearization of the tube domain in $(V / / W)_{\mathbb{R}}$ over the simplicial cone $E_{W}$ in $T_{W, \mathbb{R}}$.
\end{abstract}

\section{Contents}

$\S 1$. Parallelotopes $J_{W}^{\{\varepsilon\}}\left(\lambda^{[\varepsilon]}\right)$ and Polyhedra $K_{W}^{\varepsilon}\left(\lambda^{[\varepsilon]}\right)$ §1.1. Finite reflection group $W$

Communicated by T. Kawai. Received May 6, 2004.

2000 Mathematics Subject Classification(s): 14P10, 20F55

${ }^{\dagger}$ This article is an invited contribution to a special issue of Publications of RIMS commemorating the fortieth anniversary of the founding of the Research Institute for Mathematical Sciences.

It is an expanded version of an unpublished note of the author "The geometric generators for Artin groups of finite type (1983)", which was a sketch of the proof for the case $\varepsilon=+1$. Professor Egbert Brieskorn, at a conference Oberwolfach (1996), suggested the author to publish it. It is a great pleasure to the author to realize his suggestion at the occasion of the 40th anniversary of RIMS. The complete version including a proof of Theorem C shall appear in [S4].

* Research Institute for Mathematical Sciences, Kyoto University, Kyoto 606-8502, Japan. e-mail: saito@kurims.kyoto-u.ac.jp 
§1.2. Simplicial cone decomposition of $V_{\mathbb{R}}$

§1.3. Polyhedron dual to the simplicial cone decomposition

$\S 1.4$. Invariants for $W$ and the quotient variety $S_{W}$

§1.5. Discriminant divisor and the central component $\mathcal{C}\{\varepsilon\}$

$\S 1.6$. Primitive vector field $D$ and $\mathbb{G}_{a}$-action $\tau$ on $S_{W}$

$\S 1.7$. Opposite components $\mathcal{C}_{ \pm}^{[\varepsilon]}$ of $S_{W, \mathbb{R}}^{[\varepsilon]} \backslash D_{W, \mathbb{R}}^{[\varepsilon]}$

§1.8. Semi-algebraic polyhedra $\bar{J}_{W}^{\{\varepsilon\}}\left(\lambda^{[\varepsilon]}\right)$ in $S_{W, \mathbb{R}}^{[\varepsilon]}$ and $\bar{K}_{W}^{\varepsilon}\left(\lambda^{[\varepsilon]}\right)$ in $V_{\mathbb{R}}^{\varepsilon}$

$\S 2$. The Central Region $E_{W}^{\{\varepsilon\}}$ in $T_{W, \mathbb{R}}^{[\varepsilon]}$

$\S 2.1$. $\tau$-quotient space $T_{W}$ and $\tau$-quotient morphism $\pi_{\tau}$

§2.2. Bifurcation divisor $B_{W}=\cup_{p=2}^{\infty} B_{W, p}$

$\S 2.3$. Twisted real forms of the $\tau$-action and the $\tau$-quotient space

$\S 2.4$. Subspace $S_{W\left(I_{2}(h)\right)}$ of $S_{W}$

$\S 2.5$. V.o. axis, v.o. line and the sign factor $\sigma\left(D,\left\{\Pi_{1}, \Pi_{2}\right\}\right)$

§2.6. Algebroid functions $\varphi_{\alpha}$ and $\phi_{\alpha}$ for $\alpha \in \Pi$

$\S 3 . \quad$ Linearization Map $c_{W}$

§3.1. Linear model spaces $\widehat{V_{\Pi}}$ and $V_{\Pi}$

$\S 3.2$. $\Gamma(W)$-cone $E_{\Gamma(W)}$ in $V_{\Pi}$

$\S 3.3$. Covering spaces $T_{W, \text { odd, } \mathbb{C}}$ and $S_{W, \text { odd, }}$

$\S 3.4$. Linearization morphism $c_{W}$ on $S_{W, \text { odd, } \mathbb{C}}$

$\S 3.5$. Theorem C

$\S 3.6$. Proof of Theorem A

$\S 3.7$. Proof of Theorem B

$\S 3.8$. Examples of type $A_{3}$

$\S 4$. Fundamental Group of $S_{W, \mathbb{C}} \backslash D_{W, \mathbb{C}}$

§4.1. 1-skeleton of the polyhedron $J_{W}^{\{\varepsilon\}}(\lambda)$

$\S 4.2$. Proof of Theorem 4.1

\$4.3. Zariski-van Kampen generator system

$\S 4.4$. Proof of Theorem 4.2

\$4.5. Comparison of generator systems for $\varepsilon \in\{ \pm 1\}$

References 


\section{Table of Figures}

Fig. 1. Four base point loci in $T_{W \text {,odd, } \mathbb{C}}$

Fig. 2. The linearization maps of type $A_{3}$

Fig. 3. The generator on 1-skeleton of $J_{W}^{\{\varepsilon\}}\left(\lambda^{[\varepsilon]}\right)$

Fig. 4. The 2-facet $\left[p_{\alpha}, p_{\beta}, *\right]$

Fig. 5. Pencil close to $\overline{\alpha \beta}$-edge

Fig. 6. The Zariski-van Kampen generators on a $\tau$-orbit

Fig. 7. The complexification of the vertex orbit axis $A O$

Fig. 8. Polyhedra $J_{A_{2}}^{\{+1\}}\left(\lambda^{[+1]}\right)$ and $K_{A_{2}}^{+}\left(\lambda^{[+1]}\right)$ for $\lambda^{[+1]}=1$

Fig. 9. Polyhedra $J_{A_{2}}^{\{-1\}}\left(\lambda^{[-1]}\right)$ and $K_{A_{2}}^{-}\left(\lambda^{[-1]}\right)$ for $\lambda^{[-1]}=1$

Fig. 10. Positions of $S_{A_{2}, \mathbb{R}}^{[+1]}$ and $S_{A_{2}, \mathbb{R}}^{[-1]}$ inside $S_{A_{2}, \mathbb{C}} \cap\{\operatorname{Im}(R)=0\}$

Fig. 11. Polyhedra $J_{B_{2}}^{\{ \pm 1\}}\left(\lambda^{[ \pm 1]}\right)$ and $K_{B_{2}}^{ \pm 1}\left(\lambda^{[ \pm 1]}\right)$ for $\lambda^{[ \pm 1]}=1$

Fig. 12. Positions of $S_{B_{2}, \mathbb{R}}^{[ \pm 1]}$ and $S_{B_{2}, \mathbb{R}}^{[\beta]}$ inside $S_{B_{2}, \mathbb{C}} \cap\{\operatorname{Im}(R)=0\}$

\section{Introduction}

Let $V_{\mathbb{R}}$ be a finite-dimensional real vector space and $W$ a finite group acting irreducibly on $V_{\mathbb{R}}$ generated by reflections. We denote by $V$ the associated scheme over $\mathbb{R}$ and by $S_{W}:=V / / W$ the quotient scheme ${ }^{1}$. Let $D_{W} \subset S_{W}$ be the discriminant divisor defined by the zero locus of $\Delta:=$ the square of the fundamental anti-invariant of $W$. The open regular part $(V / / W)^{r e g}$, defined as the complement $S_{W} \backslash D_{W}$, is a simple geometric object where interests from several different areas of mathematics (e.g., Lie algebra theory, complex and differential geometries,...,etc.) intersect.

We recall two basic results on the topology of the complexification $(V / / W)_{\mathbb{C}}^{r e g}:=S_{W, \mathbb{C}} \backslash D_{W, \mathbb{C}}$ of the regular orbit space:

a) the fundamental group of $(V / / W)_{\mathbb{C}}^{r e g}$ is an Artin group (generalized braid group) (Brieskorn [Br1],[Br2] and [BS]), and

b) the universal covering of $(V / / W)_{\mathbb{C}}^{r e g}$ is contractible (Deligne [D1]).

Interestingly, for the both results, the polyhedron $K_{W}$ which is dual to the simplicial cone decomposition of $V_{\mathbb{R}}$ plays an essential role. Namely, a) the 1-skeleton and the 2-skeleton of $K_{W}$ determine the generators and relations

\footnotetext{
${ }^{1}$ We mean by " $V / / W$ " the categorical quotient scheme (1.4.5) of $V$ by the $W$-action. Even though $W$ is a finite group, it is convenient to use scheme-theoretic concepts and notation, since we study mainly over the real number field $\mathbb{R}$. The set theoretic quotient space $V_{\mathbb{R}} / W$ is not sufficient to describe structures we study. The $\mathbb{R}$ or $\mathbb{C}$-rational point set of a scheme is indicated by the subscript $\mathbb{R}$ or $\mathbb{C}$, respectively.
} 
for the fundamental group of $(V / / W)_{\mathbb{C}}^{r e g}$, and b) the contractibility of $K_{W}$ is a key step in the proof ([D1]) of the contractibility of the nerve of a simple covering of the universal covering of $(V / / W)_{\mathbb{C}}^{r e g}$. We remark further that $\left.\mathrm{c}\right)$ the dual polyhedron $K_{W}$ also describes the Stiefel-Whitney class of a related vector bundle $([\mathrm{Hu}],[\mathrm{M}]$ and $[\mathrm{N}])$.

A goal of the present paper is to reconstruct the dual polyhedron $K_{W}$ from a completely different viewpoint. The quotient variety $S_{W}:=V / / W$ carries a differential geometric structure, called the flat structure (Saito $[\mathrm{S} 1,3]$ ). Then we shall make use of a part of the real flat structure to construct the polyhedron as follows.

A principal ingredient of the flat structure is the vector field $D$ on $S_{W}$, called the primitive vector field (1.6.1), of the lowest degree, which is unique up to a constant factor. The integration $\exp (\lambda D)$ of $D$ induces a $\mathbb{G}_{a}$-action $\tau$ on $S_{W}$ (1.6.2), transversal to the discriminant divisor $D_{W}$ (see $[\mathrm{S} 2,3]$ for the role of $D$ in the theory of primitive forms).

For $\varepsilon \in\{ \pm 1\}$, consider the real form $S_{W, \mathbb{R}}^{[\varepsilon]}$ of $S_{W, \mathbb{C}}$ (the "quotient real form" of the real form $V_{\mathbb{R}}^{\varepsilon}:=\sqrt{\varepsilon} \otimes V_{\mathbb{R}}$ of $V_{\mathbb{C}}:=\mathbb{C} \otimes V$, see (1.4.8)). The $\mathbb{G}_{a}$-action $\tau$ induces the one-parameter group action $\tau^{[\varepsilon]}: \mathbb{R} \times S_{W, \mathbb{R}}^{[\varepsilon]} \rightarrow S_{W, \mathbb{R}}^{[\varepsilon]}$ (see (1.6.4)). For each fixed $\lambda^{[\varepsilon]} \in \mathbb{R}_{>0}$, consider three real hypersurfaces in $S_{W, \mathbb{R}}^{[\varepsilon]}$ : a) the real discriminant locus : $D_{W, \mathbb{R}}^{[\varepsilon]}$ and $\left.\mathrm{b}\right)^{ \pm}$the positive and negative translations of the real discriminant locus: $\tau^{[\varepsilon]}\left(+\lambda^{[\varepsilon]}\right)\left(D_{W, \mathbb{R}}^{[\varepsilon]}\right)$ and $\tau^{[\varepsilon]}\left(-\lambda^{[\varepsilon]}\right)\left(D_{W, \mathbb{R}}^{[\varepsilon]}\right)$. Then, for $\varepsilon \in\{ \pm 1\}$ and each $\lambda^{[\varepsilon]} \in \mathbb{R}_{>0}$, one has:

Theorem A (§1.8). There exists an open semi-algebraic parallelotope $J_{W}^{\{\varepsilon\}}\left(\lambda^{[\varepsilon]}\right)$ in $S_{W, \mathbb{R}}^{[\varepsilon]}$, which is surrounded ${ }^{2}$ by the hypersurfaces a) and $\left.b\right)^{ \pm}$. It is adjacent to the origin $o \in S_{W, \mathbb{R}}^{[\varepsilon]}$, and the faces adjacent to the origin are indexed by the set $\Pi$ of simple generators of $W$.

Theorem B (§1.8). The inverse image $\bar{K}_{W}^{\varepsilon}\left(\lambda^{[\varepsilon]}\right)$ in $V_{\mathbb{R}}^{\varepsilon}$ of the closure $\bar{J}_{W}^{\{\varepsilon\}}\left(\lambda^{[\varepsilon]}\right)$ of the parallelotope in $S_{W, \mathbb{R}}^{[\varepsilon]}$ is a closed semi-algebraic polyhedron which is dual to the simplicial cone decomposition of $V_{\mathbb{R}}^{\varepsilon}$ by the Weyl chambers.

See Appendix Fig. 8-12 for illustrated examples of $\bar{J}_{W}^{\{\varepsilon\}}\left(\lambda^{[\varepsilon]}\right)$ and $\bar{K}_{W}^{\varepsilon}\left(\lambda^{[\varepsilon]}\right)$ of type $A_{2}$ and $B_{2}$.

It was asked by Brieskorn, Deligne, and the author to find some descriptions of the generator system of $\pi_{1}\left(S_{W, \mathbb{C}} D_{W, \mathbb{C}}, *\right)$ as an Artin group in terms of the geometry of $S_{W}$. Let us give two answers to this question as an application of Theorems A and B (see $\S 4$ for details and proofs).

\footnotetext{
${ }^{2}$ By the word "surrounded", we mean that $J_{W}^{\{\varepsilon\}}\left(\lambda^{[\varepsilon]}\right)$ is a connected component of $S_{W, \mathbb{R}}^{[\varepsilon]} \backslash\left(D_{W, \mathbb{R}}^{[\varepsilon]} \cup \tau^{[\varepsilon]}\left(\lambda^{[\varepsilon]}\right)\left(D_{W, \mathbb{R}}^{[\varepsilon]}\right) \cup \tau^{[\varepsilon]}\left(-\lambda^{[\varepsilon]}\right)\left(D_{W, \mathbb{R}}^{[\varepsilon]}\right)\right)$.
} 
1. Let $a o^{\{\varepsilon\}}\left(\lambda^{[\varepsilon]}\right)$ be the vertex of $J_{W}^{\{\varepsilon\}}\left(\lambda^{[\varepsilon]}\right)$ antipodal to the origin $o$. Due to Theorem A, the edges of $J_{W}^{\{\varepsilon\}}\left(\lambda^{[\varepsilon]}\right)$ adjacent to $a o^{\{\varepsilon\}}\left(\lambda^{[\varepsilon]}\right)$ are indexed by the set $\Pi$ in such a manner that the $\alpha$ th edge for $\alpha \in \Pi$ intersects the $\alpha$ th face of $J_{W}^{\{\varepsilon\}}\left(\lambda^{[\varepsilon]}\right)$ transversally at a point, say $p_{\alpha}$, in $D_{W, \mathbb{R}}^{[\varepsilon]}$ (see Fig. 4). Inside a complexification of the $\alpha$ th edge (an open complex curve in $S_{W, \mathbb{C}}$ containing the $\alpha$ th edge), take a path, say $\gamma_{\alpha}$, based at $a o^{\{\varepsilon\}}\left(\lambda^{[\varepsilon]}\right)$ and turning counterclockwise once around the discriminant divisor $D_{W, \mathbb{C}}$ at $p_{\alpha}$ (Fig. 3). The class of $\gamma_{\alpha}$ in $S_{W, \mathbb{C}} \backslash D_{W, \mathbb{C}}$ is uniquely determined by the index $\alpha \in \Pi$.

Corollary 1 ( $\$ 4.1$ and $\S 4.2)$. The 1-homotopy classes of $\gamma_{\alpha}$ for $\alpha \in \Pi$ give a system of generators for $\pi_{1}\left(S_{W, \mathbb{C}} \backslash D_{W, \mathbb{C}}, a o^{\{\varepsilon\}}\left(\lambda^{[\varepsilon]}\right)\right)$, which satisfy the system of fundamental braid relations for the Artin group.

2. Next, we choose an arbitrary point $* \in J_{W}^{\{\varepsilon\}}\left(\lambda^{[\varepsilon]}\right)$ and consider the orbit $\tau^{[\varepsilon]}(\mathbb{R}) \cdot *$ which is a real line in $S_{W, \mathbb{R}}^{[\varepsilon]}$. If $*$ is generic, the real line intersects $l$ distinct points of the real discriminant locus $D_{W, \mathbb{R}}^{[\varepsilon]}$ (Fig. 5). One chooses paths inside the complex line $\tau^{[\varepsilon]}(\mathbb{C}) \cdot *$ as in Fig. 6, whose homotopy classes are called the Zariski-van Kampen generators.

Corollary 2 ( $\S 4.3$ and $\S 4.4)$. The system of the Zariski-van Kampen generators is homotopic to the generator system in Corollary 1.

Theorems A and B and their corollaries are direct applications of another basic Theorem $\mathrm{C}$ on the real bifurcation set which we explain below.

The quotient space $T_{W}:=S_{W} / / \tau$ by the $\tau$-action is a smooth $(l-1)$ dimensional affine variety. The quotient map $\pi_{\tau}: S_{W} \rightarrow T_{W}$ is a linear projection in the direction of the primitive vector field. The restriction $\pi_{\tau} \mid D_{W}$ of $\pi_{\tau}$ to the discriminant divisor is a finite covering over $T_{W}$. The ramification divisor $B_{W}$ in $T_{W}$, i.e., the discriminant divisor of the covering $\pi_{\tau} \mid D_{W}$, is called the bifurcation set. Decompose it as $B_{W}=\cup_{p=2}^{\infty} B_{W, p}$ according to the ramification index $p$, where $B_{W, 1}$ does not appear due to the transversality property of the primitive vector field $D$ to $D_{W}$. We split the bifurcation set $B_{W}$ into the ordinary part $B_{W, 2}$ and the higher part $B_{W, \geq 3}$ (called the stratum of Maxwell's convention and the caustics, respectively, in [T2]).

For each $\varepsilon \in\{ \pm 1\}$, we introduce some closed subset $O^{\varepsilon}$ in $T_{W, \mathbb{R}}^{[\varepsilon]} \backslash B_{W, \geq 3, \mathbb{R}}^{[\varepsilon]}$ (resp. $A O^{\varepsilon}$ in $S_{W, \mathbb{R}}^{[\varepsilon]} \backslash D_{W, \mathbb{R}}^{[\varepsilon]}$ ), which are homeomorphic to the real half line and are defined by the help of regular eigenvectors of the Coxeter element of $W$ (see 2.5). They shall play two basic roles: i) to single out particular connected components of $T_{W, \mathbb{R}}^{[\varepsilon]} \backslash B_{W, \geq 3, \mathbb{R}}^{[\varepsilon]}$ (resp. $S_{W, \mathbb{R}}^{[\varepsilon]} \backslash D_{W, \mathbb{R}}^{[\varepsilon]}$ ) containing them, and ii) to be chosen as a base point for the fundamental group of the complexification

\footnotetext{
${ }^{3}$ This fact is a non-trivial consequence of Theorem $\mathrm{C}$ stated below.
} 
$T_{W, \mathbb{C}} \backslash B_{W, \geq 3, \mathbb{C}}$ (resp. $S_{W, \mathbb{C}} \backslash D_{W, \mathbb{C}}$ ). On the other hand, they are related with the vertex of the polyhedra $J_{W}^{\{\varepsilon\}}\left(\lambda^{[\varepsilon]}\right)$ as: $A O^{\varepsilon}=\left\{a o^{\{\varepsilon\}}\left(\lambda^{[\varepsilon]}\right) \mid \lambda^{[\varepsilon]} \in \mathbb{R}_{>0}\right\}$ and $O^{\varepsilon}=\pi_{\tau}\left(A O^{\varepsilon}\right)$. We, therefore, call $A O^{\varepsilon}$ the half vertex orbit axis and $O^{\varepsilon}$ the half vertex orbit line (here "half" indicates that they are isomorphic to the half line $\mathbb{R}_{>0}$ ).

The connected component $\mathcal{C}^{\{\varepsilon\}}$ of $S_{W, \mathbb{R}}^{[\varepsilon]} \backslash D_{W, \mathbb{R}}^{[\varepsilon]}$ containing $A O^{\varepsilon}$, called the central component, is nothing but the image of a Weyl chamber in $V_{\mathbb{R}}^{\varepsilon}$. The connected component $E_{W}^{\{\varepsilon\}}$ of $T_{W, \mathbb{R}}^{[\varepsilon]} \backslash B_{W, \geq 3, \mathbb{R}}^{[\varepsilon]}$ containing $O^{\varepsilon}$, called the central region, is a key object in the present paper. Although the region $E_{W}^{\{\varepsilon\}}$ contains the image $\pi_{\tau}\left(\mathcal{C}^{\{\varepsilon\}}\right)$, they are different. In fact, the gap $E_{W}^{\{\varepsilon\}} \backslash \pi_{\tau}\left(\mathcal{C}^{\{\varepsilon\}}\right)$ is "growing exponentially" as the rank $l$ grows.

Theorem $\mathrm{C}$ of the present paper concerns a description of the central region $E_{W}^{\{\varepsilon\}}$ and its inverse image $\pi_{\tau}^{-1}\left(E_{W}^{\{\varepsilon\}}\right)$ (called the tube domain) in $S_{W, \mathbb{R}}^{[\varepsilon]}$.

Let $\widehat{V}_{\Pi}:=\oplus_{\alpha \in \Pi} \mathbb{R} v_{\alpha}$ be the vector space with basis $v_{\alpha}$ attached to $\alpha \in \Pi$ of simple generators for $W$, and let $V_{\Pi}:=\widehat{V}_{\Pi} / \mathbb{R} v_{\Pi}$ be the quotient space for $v_{\Pi}:=\sum_{\alpha \in \Pi} v_{\alpha}$, and let $\pi_{\Pi}: \widehat{V}_{\Pi} \rightarrow V_{\Pi}$ be the projection.

Theorem $\mathbf{C}(\S 3.5)$. $\quad$ There exist $i)$ an open simplicial cone $E_{\Gamma(W)} \subset V_{\Pi}$ depending only on the Coxeter diagram $\Gamma(W)$ in such a manner that its faces are indexed by the edges of $\Gamma(W)$, and ii) real algebroid maps $c_{W}$ and $b_{W}$ with the commutative diagram:

$$
\begin{array}{ccc}
\left(\pi_{\tau}^{[\varepsilon]}\right)^{-1}\left(E_{W}^{\{\varepsilon\}}\right) & \stackrel{c_{W}}{\simeq}\left(\pi_{\Pi}\right)^{-1}\left(E_{\Gamma(W)}\right) \\
\pi_{\tau}^{[\varepsilon]}{ } & & \pi_{\Pi} \downarrow \\
E_{W}^{\{\varepsilon\}} & \stackrel{b_{W}}{\simeq} & E_{\Gamma(W)}
\end{array}
$$

where we mean by $\simeq$ a semi-algebraic isomorphism. The map $c_{W}$ induces a bijection

$$
D_{W, \mathbb{R}}^{[\varepsilon]} \cap\left(\pi_{\tau}^{[\varepsilon]}\right)^{-1}\left(E_{W}^{\{\varepsilon\}}\right) \simeq\left(\cup_{\alpha \in \Pi} H_{\alpha}\right) \cap\left(\pi_{\Pi}\right)^{-1}\left(E_{\Gamma(W)}\right)
$$

where $H_{\alpha}$ is the coordinate hyperplane in $\widehat{V}_{\Pi}$ w.r.t. the $\alpha$ th coordinate.

The linearization maps $c_{W}$ and $b_{W}$ of type $A_{3}$ are illustrated in Fig. 2. Precise statements of Theorems A, B and $\mathrm{C}$ are given in $\S 1.8$ and $\S 3.5$.

Theorems A and B and their corollaries are proved in $\S 3$ as the direct consequences of Theorem C. However, Theorem $\mathrm{C}$ is not proved in the present article, since Theorem $\mathrm{C}$ is a part of consequences of a general study of the linearization maps $c_{W}$ and $b_{W}$, whose comprehensive treatment shall appear in [S4]. 
Before we go further, we explain a motivation of the present paper. The quotient variety $S_{W}$ appears as the base space of the universal unfolding $X_{W} \rightarrow$ $S_{W}$ of a simple singularity [Br3]. On the total space $X_{W}$ there is a special de Rham cohomology class relative to $S_{W}$, called the primitive form $\zeta_{W}^{(0)}$ [S2]. The period integral $\int \zeta_{W}^{(0)}$ over cycles in the fibers of the unfolding gives a multivalued map, called the period map, defined on $S_{W, \mathbb{C}} \backslash D_{W, \mathbb{C}}$ to the period domain. For the study of the period map, we need to understand the homotopy groups of the space $S_{W, \mathbb{C}} \backslash D_{W, \mathbb{C}}$. This gives one motivation.

The primitive form induces the flat structure on $S_{W}$, where $\zeta_{W}^{(0)}$ is identified with the primitive vector field $D$ on $S_{W}$ ([S2]). In the present paper, we employ not only $D$ but the basic framework of the theory of primitive forms such as the $\tau$-orbit space $T_{W}$ with its bifurcation divisor $B_{W}$, the characteristic variety $C_{W}$ and the finite morphism $q_{W}: C_{W} \rightarrow T_{W}$. The vertex orbit axis $A O$ is nothing but the real form of the coordinate axis for the lowest degree flat coordinate $P_{1}$. Therefore, it does not seem an accident that the polyhedron $K_{W}$ is reconstructed through the action $\tau$, the integral of the primitive vector field. However, we still need to clarify the relation of the period map for $\zeta_{W}^{(0)}$ with the polyhedron $K_{W}(\lambda)$. Some natural questions are the followings. Can one reconstruct Deligne's proof [D1] in terms of the semi-algebraic geometry of the spaces $V$ and $V / / W$ as in the present work? Is $T_{W, \mathbb{C}} \backslash B_{W, \geq 3, \mathbb{C}}$ an Eilenberg-MacLane space? Determine the fundamental relations for its fundamental group with respect to the natural generators indexed by the edges of $\Gamma(W)$.

There are many precedent works on the semi-algebraic geometry of the space $S_{W}$ with the discriminant divisor $D_{W}$ in it, among others, by Hilbert $[\mathrm{H}]$, Thom [T1,2], Arnold [Ar1,2], Looijenga [Lo1,2], Springer [Sp1,2] and Tits. In particular, Thom's idea on the universal unfolding ([T2]) influenced either directly or indirectly on the idea of the primitive form and the primitive vector field. We also note an article on the semi-algebraic geometry of the orbit spaces of compact Lie groups by Procesi-Schwarz [P-S], though we do not know yet its direct relation with the present paper.

Let us explain the construction of the present paper.

The first half of $\S 1$ is an elementary preparation on the quotient variety $S_{W}:=V / / W$ by the finite reflection group $W$. Then, we introduce the $\tau$-action on $S_{W}$ and on its real forms. After these preparations, we formulate Theorems $\mathrm{A}$ and $\mathrm{B}$ in $\S 1.8$.

$\S 2$ studies the $\tau$-quotient variety $T_{W}$ with its bifurcation set $B_{W}$. After introducing the base point loci $O^{\varepsilon}$ in $T_{W, \mathbb{C}} \backslash B_{W, \geq 3, \mathbb{C}}$, we introduce the central 
regions $E_{W}^{\{\varepsilon\}}$ in $\S 2.5$, and algebroid functions $\varphi_{\alpha, \varepsilon}$ in $\S 2.6$. This section is an extract from $\S 2-\S 9$ of the forthcoming paper [S4]. Leaving a general treatment to $[\mathrm{S} 4]$, we restrict our attention only to the real structures $[\varepsilon]$ for $\varepsilon= \pm 1$. We also omitted the study of the characteristic variety $C_{W}$ (which plays an important role in [S4] to understand the discriminant divisor $D_{W}$ ).

In $\S 3$, we study the linearization map $c_{W}$. The target spaces $\widehat{V}_{\Pi}, V_{\Pi}$ and the simplicial cone $E_{\Gamma(W)}$ are introduced in $\S 3.1$ and $\S 3.2$. The map $c_{W}$ is introduced as an algebroid map in $\S 3.4$. Using them, Theorem $\mathrm{C}$ is formulated in $\S 3.5$. As its application, Theorems A and B are proved in $\S 3.6$ and $\S 3.7$. The proof of Theorem $\mathrm{C}$ is not given in the present paper but it is given in [S4], where we formulate $c_{W}$ as an algebraic correspondence, which is more appropriate for our purpose.

$\S 4$ studies the generator systems of the fundamental group of the space $S_{W, \mathbb{C}} \backslash D_{W, \mathbb{C}}$. A pair of generator systems depending on $\varepsilon \in\{ \pm 1\}$ is constructed by use of the polyhedra $J_{W}^{\{\varepsilon\}}\left(\lambda^{[\varepsilon]}\right)$ in $\S 4.1$ and is identified with Brieskorn's generator system in $§ 4.2$. A pair of the Zariski-van Kampen generator systems depending on $\varepsilon$ by the use of $\tau$-pencil is described in $\S 4.3$. It is identified in $\S 4.4$ with the one in $\S 4$.1. The relationship between the generator systems for $\varepsilon=+1$ and for $\varepsilon=-1$ is given in $\S 4.5$.

Appendix studies the rank two case in detail. The polyhedra $J_{W}^{\{\varepsilon\}}\left(\lambda^{[\varepsilon]}\right)$ and $K_{W}^{\varepsilon}\left(\lambda^{[\varepsilon]}\right)$ of types $A_{2}$ and $B_{2}$ are illustrated in Fig. 8, 9 and 11.

Concluding Remarks: The study of the polyhedra $J_{W}^{\{\varepsilon\}}\left(\lambda^{[\varepsilon]}\right), K_{W}^{\varepsilon}\left(\lambda^{[\varepsilon]}\right)$ and the real region $E_{W}^{\{\varepsilon\}}$ has just started. The proofs are rather involved. On the other hand, we have observed a new aspect of the geometry of $V, V / / W$ and $V / / W / / \tau$ : the interaction between the semi-algebraic geometry of their real forms and the topology of their complexification, where the flat structure combines them. We may briefly summarize the present work as a combinatorial aspect of the flat structure on the quotient variety by a finite reflection group. These new features of the geometry seem to the author quite attractive and worthwhile to be studied further. Perhaps (and hopefully), the study in the present paper is the first fortunate model case ${ }^{4}$ of a certain new mathematical research subject.

The author would like to express his hearty gratitude to Professors Masaki Kashiwara and Takahiro Kawai for their supports and helps during the preparation of the present paper, to Professor Hiroaki Terao for careful reading of the

\footnotetext{
${ }^{4}$ One next model may be the case of elliptic root systems, which admit again the flat structure. Since the complement of the complex discriminant divisor may have 2-homotopy classes, we need to study the non-simply connected polyhedra.
} 
manuscripts and many useful pieces of advice, and to Mrs. Kumiko Matsumura for her beautiful drawing of figures.

The author would like to express his deep sorrow to the early death of the late Professor Nobuo Sasakura (March 5, 1941 - June 16, 1997), who constantly showed interests in the present work when it was in a preparatory form.

\section{§1. Parallelotopes $J_{W}^{\{\varepsilon\}}\left(\lambda^{[\varepsilon]}\right)$ and Polyhedra $K_{W}^{\varepsilon}\left(\lambda^{[\varepsilon]}\right)$}

We construct the main objects $J_{W}^{\{\varepsilon\}}\left(\lambda^{[\varepsilon]}\right)$ and $K_{W}^{\varepsilon}\left(\lambda^{[\varepsilon]}\right)$ of the present paper, and give precise statements of Theorems A and B announced in the introduction.

In 1.1-1.5, we recall basic results on a finite reflection group $W$ and its invariants from [B, Ch.4,5]. In 1.6 and 1.7, we introduce the new concept: the $\tau$-action on the $W$-quotient varieties $S_{W, \mathbb{R}}^{[ \pm 1]}$. By the use of the $\tau$-action, Theorems $\mathrm{A}$ and $\mathrm{B}$ in $\S 1.8$ describe the polyhedra $J_{W}^{\{\varepsilon\}}\left(\lambda^{[\varepsilon]}\right)$ and $K_{W}^{\varepsilon}\left(\lambda^{[\varepsilon]}\right)$.

\section{§1.1. $\quad$ Finite reflection group $W$}

Let $V_{\mathbb{R}}$ be an $\mathbb{R}$-vector space of rank $l$ equipped with the classical topology. An element $\alpha \in \operatorname{GL}\left(V_{\mathbb{R}}\right)$ is a reflection if there exist $e_{\alpha} \in V_{\mathbb{R}}$ and $f_{\alpha} \in V_{\mathbb{R}}^{*}:=$ $\operatorname{Hom}_{\mathbb{R}}\left(V_{\mathbb{R}}, \mathbb{R}\right)$ with $\left\langle f_{\alpha}, e_{\alpha}\right\rangle=2$ such that $\alpha(x)=x-f_{\alpha}(x) e_{\alpha}$ for $x \in V_{\mathbb{R}}$. Two vectors $e_{\alpha}$ and $f_{\alpha}$ are not unique but $e_{\alpha} \otimes f_{\alpha}$ is uniquely determined by $\alpha$. If $I$ is an $\alpha$-invariant symmetric bilinear form on $V_{\mathbb{R}}$ such that $I\left(e_{\alpha}, e_{\alpha}\right) \neq 0$, then $f_{\alpha}(x)=I\left(e_{\alpha}^{\vee}, x\right)$ for $e_{\alpha}^{\vee}:=2 e_{\alpha} / I\left(e_{\alpha}, e_{\alpha}\right)$. The kernel $H_{\alpha}:=\operatorname{ker}\left(f_{\alpha}\right)=\operatorname{ker}(1-\alpha)$ is called the reflection hyperplane of $\alpha$.

Let $W$ be a finite group generated by reflections on $V_{\mathbb{R}}$ and $I$ a $W$-invariant positive-definite symmetric bilinear form on $V$. Assume that $W$ acts irreducibly on $V_{\mathbb{R}}$. Then, $I$ is unique up to a positive constant factor. Put

$$
R(W):=\{\alpha \in W \mid \alpha \text { is a reflection }\} .
$$

We recall some basic facts on $W$ in $[\mathrm{B}]$.

1. A connected component of $V_{\mathbb{R}} \backslash \cup_{\alpha \in R(W)} H_{\alpha}$, called a Weyl chamber, is a simplicial cone. The group $W$ acts simply transitively on the set of chambers.

2. For a chamber $C$, put $\Pi(C):=\left\{\alpha \in R(W) \mid H_{\alpha}\right.$ is a wall of $\left.C\right\}$ Then $(W, \Pi(C))$ is a Coxeter system with respect to the Coxeter matrix $M_{W}:=$ $\left(m_{\alpha \beta}\right)_{\alpha, \beta \in \Pi(C)}$ with $m_{\alpha \beta}:=\operatorname{ord}(\alpha \beta)$ (see [B, Ch.IV, $\left.\left.\S 1 \mathrm{n}^{o} 1.3.\right]\right)$.

3. The closure $\bar{C}$ of a chamber $C$ is a fundamental domain of the action of $W$ on $V_{\mathbb{R}}$, that is, $\bar{C} \rightarrow V_{\mathbb{R}} / W$ is a homeomorphism.

4. The vectors $\left\{e_{\alpha} \mid \alpha \in \Pi(C)\right\}$ form a basis of $V_{\mathbb{R}}$. Choose $e_{\alpha}$ so that $C=$ $\left\{x \in V_{\mathbb{R}} \mid\left\langle f_{\alpha}, x\right\rangle>0\right.$ for $\left.\alpha \in \Pi(C)\right\}$. Then i) $I\left(e_{\alpha}, e_{\beta}\right) \leq 0$ for $\alpha \neq \beta \in \Pi(C)$, 
and ii) the coefficients of the expression $e_{\gamma}=\sum_{\alpha \in \Pi(C)} c_{\alpha} e_{\alpha}$ for any $\gamma \in R(W)$ are either all non-negative or all non-positive.

\section{§1.2. Simplicial cone decomposition of $V_{\mathbb{R}}$}

For a subset $F \subset R(W)$, consider the subspace $H_{F}:=\cap_{\beta \in F} H_{\beta}$ of $V_{\mathbb{R}}$ and the set of hyperplanes of $H_{F}$ induced by reflection hyperplanes:

$$
A\left(H_{F}\right):=\left\{H_{F} \cap H_{\alpha} \mid \alpha \in R(W), H_{\alpha} \not \supset H_{F}\right\} .
$$

A point in $H_{F}$ is called generic if it lies in $\dot{H}_{F}:=H_{F} \backslash \cup_{G \in A\left(H_{F}\right)} G$. A connected component of $\dot{H}_{F}$ is called a facet of $V_{\mathbb{R}}$. Let $\Gamma$ be the index set of all facets of $V_{\mathbb{R}}$ by running all finite subset $F$ of $R(W)$, and let us denote by $V_{\gamma}$ the facet corresponding to $\gamma \in \Gamma$. Then the vector space $V_{\mathbb{R}}$ decomposes into a disjoint union of facets:

$$
V_{\mathbb{R}}=\sqcup_{\gamma \in \Gamma} V_{\gamma} .
$$

Put $\gamma \leq \delta$ for $\gamma, \delta \in \Gamma$ iff $V_{\gamma} \subset \bar{V}_{\delta}$. The decomposition is a stratification, i.e., it satisfies the boundary condition: if $V_{\gamma} \cap \bar{V}_{\delta} \neq \emptyset$ then $V_{\gamma} \subset \bar{V}_{\delta}$. The minimal element of $\Gamma$ is denoted by 0 (i.e., $V_{0}=\{0\}$ ). The maximal elements of $\Gamma$ correspond to chambers. Any stratum is a cone over a simplex, and hence (1.2.2) is called the simplicial cone decomposition of $V_{\mathbb{R}}$.

\section{§1.3. Polyhedron dual to the simplicial cone decomposition}

Definition. 1. A compact subset $\bar{P}$ in $\mathbb{R}^{l}$ with a fixed semi-algebraic stratification (a finite decomposition of $\bar{P}$ into smooth semi-algebraic sets satisfying the boundary condition) is called a semi-algebraic polyhedron, if there is a semi-algebraic diffeomorphism, say $\varphi$, from $\bar{P}$ to a polyhedron in $\mathbb{R}^{l}$ (a convex hull of finite points in $\mathbb{R}^{l}$ which has non-trivial interior points). More precisely, $\varphi$ induces an isomorphism from each stratum to a facet of the polyhedron. A stratum of $\bar{P}$ corresponding to a face, facet or vertex is called a face, facet or vertex of $\bar{P}$, respectively. The set $P$ of interior points of $\bar{P}$ is called an open semi-algebraic polyhedron. We say the faces of $\bar{P}$ are crossing normally at a point $x \in \bar{P}$, if there is a real-analytic diffeomorphism from a neighborhood of $x$ in $\mathbb{R}^{l}$ to a neighborhood of the origin of $\mathbb{R}^{l}$ which maps locally $(\bar{P}, x)$ to $\left(\mathbb{R}_{\geq 0}^{k} \times \mathbb{R}^{l-k}, 0\right)$ for some $0 \leq k \leq l$.

2. A semi-algebraic polyhedron $\bar{K}$ in $V_{\mathbb{R}}$ is called dual to the simplicial cone decomposition (1.2.2), if it has the facet decomposition:

$$
\bar{K}=\sqcup_{\gamma \in \Gamma} K_{\gamma}
$$

indexed by the same index set $\Gamma$ as in (1.2.2) such that 
i) $\bar{K}_{\gamma} \supset K_{\delta}$ if and only if $\gamma \leq \delta$,

ii) $K_{\gamma} \cap V_{\delta} \neq \emptyset$ if and only if $\gamma \leq \delta$, for any $\gamma, \delta \in \Gamma$,

iii) if $\gamma \leq \delta$, then $K_{\gamma}$ and $V_{\delta}$ intersects transversally at each point of $K_{\gamma} \cap V_{\delta}$. There exists a real analytic diffeomorphism from a neighborhood of $\overline{K_{\gamma} \cap V_{\delta}}$ to a neighborhood of the cube $[0,1]^{k}$ of dimension $k:=\operatorname{dim}\left(V_{\delta}\right)-\operatorname{dim}\left(V_{\gamma}\right)$, which induces a homeomorphism from $\overline{K_{\gamma} \cap V_{\delta}}$ to $[0,1]^{k}$. In particular, $\operatorname{dim}\left(K_{\gamma}\right)+$ $\operatorname{dim}\left(V_{\gamma}\right)=l$ for $\gamma \in \Gamma$.

The last condition iii) implies the following property:

iv) the faces of $\bar{K}$ are crossing normally everywhere on $\bar{K}$.

The above definition implies that $K_{0}$ is an open cell in $V_{\mathbb{R}}$ containing $0 \in V_{\mathbb{R}}$ such that $K_{0}$ is the interior of $\bar{K}$. The simpliciality of the cone decomposition (1.2.2) implies that $\bar{K}$ is a manifold with corners.

\section{\$1.4. Invariants for $W$ and the quotient variety $S_{W}$}

We recall basic facts on $W$-invariants $S\left(V_{\mathbb{R}}^{*}\right)^{W}([\mathrm{~B}$, Ch.v, $\S 5])$ and fix notation on the $W$-quotient variety.

1. A product $c:=\Pi_{\alpha \in \Pi(C)} \alpha$ is called a Coxeter element. Its conjugacy class in $W$ is independent of the order of the product. The order $h$ of $c$ is called the Coxeter number. The eigenvalues of $c$ are given by $\exp \left(2 \pi \sqrt{-1} m_{i} / h\right)$ $(i=1, \ldots, l)$ where $0<m_{i}<h$ are called the exponents of $W$ and are ordered as $m_{1}=1<m_{2} \leq \cdots \leq m_{l-1}<m_{l}=h-1$.

2. Let $S\left(V_{\mathbb{R}}^{*}\right)$ be the symmetric tensor algebra of $V_{\mathbb{R}}^{*}$. We denote by $S\left(V_{\mathbb{R}}^{*}\right)^{W}$ the subring consisting of $W$-invariants in $S\left(V_{\mathbb{R}}^{*}\right)$. Chevalley's Theorem $[\mathrm{Ch}]$ states that $S\left(V_{\mathbb{R}}^{*}\right)^{W}$ is generated by $l$ algebraically independent homogeneous elements of degrees $m_{i}+1(i=1, \ldots, l)$. In the rest of the paper, we fix a homogeneous generator system $\left(P_{1}, \ldots, P_{l}\right)$ with $d_{i}:=\operatorname{deg} P_{i}=m_{i}+1$. Therefore, we have $S\left(V_{\mathbb{R}}^{*}\right)^{W} \simeq \mathbb{R}\left[P_{1}, \ldots, P_{l}\right]$.

3. The module of anti-invariants $S\left(V_{\mathbb{R}}^{*}\right)^{-W}:=\left\{P \in S\left(V_{\mathbb{R}}^{*}\right) \mid g(P)=\operatorname{det}(g)^{-1} P\right.$ for all $g \in W\}$ is a free $S\left(V_{\mathbb{R}}^{*}\right)^{W}$-module of rank one generated by

$$
\delta_{W}:=\prod_{\alpha \in R(W)} f_{\alpha} .
$$

The Jacobian of the generator system $\left(P_{1}, \ldots, P_{l}\right)$ of invariants with respect to a linear coordinates $\left(X_{1}, \ldots, X_{l}\right)$ of $V_{\mathbb{R}}$ is a basic anti-invariant:

$$
\operatorname{det}\left(\frac{\partial\left(P_{1}, \ldots, P_{l}\right)}{\partial\left(X_{1}, \ldots, X_{l}\right)}\right)=c \delta_{W} \quad \text { for } \quad c \in \mathbb{R}_{\neq 0} .
$$

4. Let $\Omega:=\exp (\pi \sqrt{-1} / h)$ be a primitive $(2 h)$ th root of unity. The eigenvector $\xi$ of a Coxeter element belonging to the eigenvalue $\Omega^{2}$ in the complexification $V_{\mathbf{C}}=\mathbb{C} \otimes V_{\mathbb{R}}$ is regular, i.e., $\delta_{W}(\xi) \neq 0([\mathrm{~B}, \mathrm{Ch} . \mathrm{V}, \S 6])$. This implies 
an equality (c.f. $\S 2.4$ Fact 1$)$ :

$$
\# R(W)=h \cdot l / 2
$$

5. The square $\Delta_{W}:=\delta_{W}^{2}$ is a $W$-invariant called the discriminant. Express $\Delta_{W}$ as a polynomial in $P_{l}$. In view of the degree counting: $\operatorname{deg}\left(P_{l}\right)=h$ and $\operatorname{deg}(\Delta)=h l$, we know that it is of the form:

$$
\Delta_{W}=A_{0} P_{l}^{l}+A_{1} P_{l}^{l-1}+\cdots+A_{l}
$$

where $A_{i}$ is a homogeneous polynomial of degree $i h$ in $P_{1}, \ldots, P_{l-1}$. Then $A_{0} \neq 0$ (since, by the degree condition, one has $P_{1}(\xi)=\ldots=P_{l-1}(\xi)=0$. Then $\Delta_{W}(\xi) \neq 0$ implies $A_{0} \neq 0$ and $\left.P_{l}(\xi) \neq 0\right)$.

6 . The categorical quotient variety $V / / W$ as a scheme over $\mathbb{R}$ is denoted by

$$
S_{W}:=V / / W:=\operatorname{Spec}\left(S\left(V_{\mathbb{R}}^{*}\right)^{W}\right),
$$

and its $\mathbb{C}$-rational point set is given by

$$
S_{W, \mathbb{C}}:=\operatorname{Hom}_{\mathbb{R}}^{\text {alg }}\left(S\left(V_{\mathbb{R}}^{*}\right)^{W}, \mathbb{C}\right)=\operatorname{Hom}_{\mathbb{C}}^{\text {alg }}\left(S\left(V_{\mathbb{C}}^{*}\right)^{W}, \mathbb{C}\right),
$$

where Hom ${ }^{a l g}$ is the set of algebra homomorphisms. The image in $S_{W, \mathbb{C}}$ of the origin 0 of $V_{\mathbb{C}}$ is denoted by $o$ and is called the origin of $S_{W, \mathbb{C}}$.

For $\varepsilon \in\{ \pm 1\}$, we consider the real form $V_{\mathbb{R}}^{\varepsilon}$ of $V_{\mathbb{C}}:=V_{\mathbb{R}} \otimes_{\mathbb{R}} \mathbb{C}$ where

$$
V_{\mathbb{R}}^{+1}:=V_{\mathbb{R}} \quad \text { and } \quad V_{\mathbb{R}}^{-1}:=\sqrt{-1} V_{\mathbb{R}}
$$

The $\mathbb{C}$-linear $W$-action on $V_{\mathbb{C}}$ leaves the real forms invariant such that $S\left(\left(V_{\mathbb{R}}^{\varepsilon}\right)^{*}\right)^{W} \otimes_{\mathbb{R}} \mathbb{C} \simeq S\left(V_{\mathbb{C}}^{*}\right)^{W}$. Thus, we introduce two real forms of $S_{W, \mathbb{C}}$ :

$$
S_{W, \mathbb{R}}^{[\varepsilon]}:=\operatorname{Hom}_{\mathbb{R}}^{a l g}\left(S\left(\left(V_{\mathbb{R}}^{\varepsilon}\right)^{*}\right)^{W}, \mathbb{R}\right)
$$

for $\varepsilon \in\{ \pm 1\}$. These two real forms coincide if $-\operatorname{id}_{V_{\mathbb{R}}} \in W$. Note that a real coordinate system of $S_{W, \mathbb{R}}^{[\varepsilon]}$ is given by $\left(P_{i} / \sqrt{\varepsilon^{m_{i}+1}}\right)_{i=1}^{l}$ so that

$$
\left(P_{1} / \sqrt{\varepsilon^{2}}, \ldots, P_{l} / \sqrt{\varepsilon^{h}}\right): S_{W, \mathbb{R}}^{[\varepsilon]} \stackrel{\sim}{\longrightarrow} \mathbb{R}^{l},
$$

where we put $\sqrt{1}:=1$ and $\sqrt{-1}:=$ the unit of pure imaginary number.

7. For any point $x \in V_{\mathbb{C}}$, the evaluation homomorphism: $S\left(V_{\mathbb{C}}\right)^{W} \ni P \mapsto$ $P(x) \in \mathbb{C}$ induces the $W$-invariant morphisms:

$$
\pi_{W, \mathbb{C}}: V_{\mathbb{C}} \rightarrow S_{W, \mathbb{C}} \quad \text { and } \quad \pi_{W, \mathbb{R}}^{\varepsilon}: V_{\mathbb{R}}^{\varepsilon} \rightarrow S_{W, \mathbb{R}}^{[\varepsilon]}(\varepsilon \in\{ \pm 1\})
$$


These morphisms are finite and closed maps with respect to the classical topology. The morphism $\pi_{W, \mathbb{C}}$ induces a homeomorphism $V_{\mathbb{C}} / W \simeq S_{W, \mathbb{C}}$, and $\pi_{\mathbb{R}}^{\varepsilon}$ induces an embedding $V_{\mathbb{R}}^{\varepsilon} / W \subset S_{W, \mathbb{R}}^{[\varepsilon]}$ onto a closed semi-algebraic set, called the central component (see Assertion 1.1 (4)).

\section{§1.5. Discriminant divisor and the central component $\mathcal{C}^{\{\varepsilon\}}$}

The discriminant divisor $D_{W}$ in $S_{W}$ is defined by $\Delta_{W}=0$. Its $\mathbb{C}$-rational point set in $S_{W, \mathbb{C}}$ or $\mathbb{R}$-rational point set in $S_{W, \mathbb{R}}^{[\varepsilon]}$ for $\varepsilon \in\{ \pm 1\}$ (called the complex or real discriminant locus) are given by

$$
D_{W, \mathbb{C}}:=\left\{t \in S_{W, \mathbb{C}} \mid \Delta_{W}(t)=0\right\} \text { and } D_{W, \mathbb{R}}^{[\varepsilon]}:=D_{W, \mathbb{C}} \cap S_{W, \mathbb{R}}^{[\varepsilon]} .
$$

The equalities (1.4.1) and (1.4.2) imply:

i) The critical values of $\pi_{W, \mathbb{C}}$ lie in the discriminant divisor $D_{W, \mathbb{C}}$.

ii) The inverse image $\pi_{W, \mathbb{C}}^{-1} D_{W, \mathbb{C}}$ is the union $\bigcup_{\alpha \in R(W)} H_{\alpha, \mathbb{C}}$.

Assertion 1.1. (1) The stabilizer subgroup of $W$ at any point $x \in V_{\mathbb{C}}$ is generated by the reflections whose reflection hyperplanes contain $x$.

(2) The complement of the discriminant locus $S_{W, \mathbb{C}} \backslash D_{W, \mathbb{C}}$ is the space of regular (i.e., stabilizer free) orbits of the $W$-action on $V_{\mathbb{C}}$.

(3) $\pi_{W, \mathbb{C}}: V_{\mathbb{C}} \backslash \cup_{\alpha \in R(W)} H_{\alpha, \mathbb{C}} \rightarrow S_{W, \mathbb{C}} \backslash D_{W, \mathbb{C}}$ is a normal covering whose covering transformation group is $W$.

(4) For $\varepsilon \in\{ \pm 1\}$, there exists a connected component $\mathcal{C}^{\{\varepsilon\}}$ of $S_{W, \mathbb{R}}^{[\varepsilon]} \backslash D_{W, \mathbb{R}}^{[\varepsilon]}$ such that for any connected component (chamber) $C$ of $V_{\mathbb{R}} \backslash \cup_{\alpha \in R(W)} H_{\alpha}$, the morphism $\pi_{W, \mathbb{R}}^{\varepsilon}$ induce the homeomorphisms:

$$
\sqrt{\varepsilon} C \simeq \mathcal{C}^{\{\varepsilon\}} \quad \text { and } \quad \sqrt{\varepsilon C} \simeq \overline{\mathcal{C}}^{\{\varepsilon\}} .
$$

We call $\mathcal{C}^{\{\varepsilon\}}$ the central component of $S_{W, \mathbb{R}}^{[\varepsilon]} \backslash D_{W, \mathbb{R}}^{[\varepsilon]}$.

(5) As a consequence of (4), $\overline{\mathcal{C}}^{\{\varepsilon\}}$ is a semi-algebraic simplicial cone with the vertex at $o$, whose faces are indexed by $\Pi=\Pi(C)$.

\section{§1.6. Primitive vector field $D$ and $\mathbb{G}_{a}$-action $\tau$ on $S_{W}$}

We fix a particular vector field $D$ on $S_{W}$, which we shall call the primitive vector field $([\mathrm{S} 3,(2.2)])$. The vector field $D$ is transversal to the discriminant divisor $D_{W}$ and plays a basic role throughout the present paper.

Let $\operatorname{Der}_{S_{W}}$ be the module of derivations of the algebra $S\left(V_{\mathbb{R}}^{*}\right)^{W}$ over $\mathbb{R}$, which is a graded $S\left(V_{\mathbb{R}}^{*}\right)^{W}$-module. Using the generator system $P_{1}, \ldots, P_{l}$ for $S\left(V_{\mathbb{R}}^{*}\right)^{W}$ (see 2 . of $\S 1.4$ ), its free basis are given by $\partial_{P_{i}}(i=1, \ldots, l)$ with $\partial_{P_{i}} P_{j}=$ $\delta_{i j}$ and $\operatorname{deg}\left(\partial_{P_{i}}\right)=-\operatorname{deg}\left(P_{i}\right)$. The maximality $\operatorname{deg}\left(P_{l}\right)>\operatorname{deg}\left(P_{i}\right)$ for $i=$ $1, \ldots, l-1$, implies that the lowest graded piece of $D e r_{S_{W}}$ is a vector space of 
dimension one spanned by

$$
D:=\partial_{P_{l}}
$$

In the rest of the paper, we fix a basis (1.6.1) and call it the primitive vector field. The primitive vector field is one of the basic building blocks for the flat structure on $S_{W}$, but we do not go into details ([S1,3]).

Integrating $D$, we introduce a group action

$$
\tau: \mathbb{G}_{a} \times S_{W} \longrightarrow S_{W}
$$

whose co-action $\tau^{*}$ on $S\left(V_{\mathbb{R}}^{*}\right)^{W}$ is given by

$$
\begin{gathered}
\tau^{*}: S\left(V_{\mathbb{R}}^{*}\right)^{W} \longrightarrow S\left(V_{\mathbb{R}}^{*}\right)^{W} \otimes \mathbb{R}[\lambda], \\
P_{i} \mapsto P_{i}(i=1, \ldots, l-1) \quad \text { and } \quad P_{l} \mapsto P_{l}+\lambda .
\end{gathered}
$$

Note that $(\tau(\mathbb{C}) \cdot o) \cap D_{W, \mathbb{C}}=\{o\}$ where $o$ is the origin of $S_{W, \mathbb{C}}$, since the leading coefficient $A_{0}$ in (1.4.4) does not vanish.

For each $\varepsilon \in\{ \pm 1\}$, let us choose and fix the real valued function

$$
\lambda^{[\varepsilon]}:=\lambda / \sqrt{\varepsilon^{h}}
$$

on the real form $\mathbb{G}_{a}^{\varepsilon^{h}}:=\sqrt{\varepsilon^{h}} \mathbb{R} \subset \mathbb{G}_{a, \mathbb{C}}=\mathbb{C}$ as its real coordinate. Then, recalling (1.4.9), one obtains the real one-parameter group action:

$$
\begin{gathered}
\tau^{[\varepsilon]}: \quad \mathbb{R} \times S_{W, \mathbb{R}}^{[\varepsilon]} \rightarrow \quad S_{W, \mathbb{R}}^{[\varepsilon]} \\
\lambda^{[\varepsilon]} \times\left(P_{1} / \sqrt{\varepsilon^{2}}, \ldots, P_{l} / \sqrt{\varepsilon^{h}}\right) \mapsto\left(P_{1} / \sqrt{\varepsilon^{2}}, \ldots, P_{l} / \sqrt{\varepsilon^{h}}+\lambda^{[\varepsilon]}\right) .
\end{gathered}
$$

A domain in $S_{W, \mathbb{R}}^{[\varepsilon]}$ is called a tube domain if it is $\tau^{[\varepsilon]}$-invariant.

§1.7. Opposite components $\mathcal{C}_{ \pm}^{[\varepsilon]}$ of $S_{W, \mathbb{R}}^{[\varepsilon]} \backslash D_{W, \mathbb{R}}^{[\varepsilon]}$

Since the half lines $\tau^{[\varepsilon]}\left(\mathbb{R}_{>0}\right) \cdot o$ and $\tau^{[\varepsilon]}\left(-\mathbb{R}_{>0}\right) \cdot o$ do not intersect the discriminant locus, we have the following definition.

Definition. The opposite components of $S_{W, \mathbb{R}}^{[\varepsilon]} \backslash D_{W, \mathbb{R}}^{[\varepsilon]}$ are

$$
\begin{aligned}
& \mathcal{C}_{+}^{[\varepsilon]}:=\text { the connected component which contains } \tau^{[\varepsilon]}\left(\mathbb{R}_{>0}\right) \cdot o \\
& \mathcal{C}_{-}^{[\varepsilon]}:=\text { the connected component which contains } \tau^{[\varepsilon]}\left(\mathbb{R}_{<0}\right) \cdot o .
\end{aligned}
$$

One has: $\mathcal{C}_{+}^{[\varepsilon]} \neq \mathcal{C}^{\{\varepsilon\}} \neq \mathcal{C}_{-}^{[\varepsilon]}$ (except for type $A_{1}$ ), since the eigenvectors for $\exp (2 \pi \sqrt{-1} / h)$ of the Coxeter element do not belong to $V_{\mathbb{R}}^{\varepsilon}$. Each of the opposite components $\mathcal{C}_{ \pm}^{[\varepsilon]}$ is the interior of the quotient of a certain twisted real 
form of $V_{\mathbb{C}}$. We shall give another expression of opposite components in (2.5.5) by determining the twisted real form.

§1.8. Semi-algebraic polyhedra $\bar{J}_{W}^{\{\varepsilon\}}\left(\lambda^{[\varepsilon]}\right)$ in $S_{W, \mathbb{R}}^{[\varepsilon]}$ and $\bar{K}_{W}^{\varepsilon}\left(\lambda^{[\varepsilon]}\right)$ in $V_{\mathbb{R}}^{\varepsilon}$ We state Theorem A announced in the introduction.

Theorem A. For $\lambda^{[\varepsilon]} \in \mathbb{R}_{>0}$ and for $\varepsilon \in\{ \pm 1\}$, put

$$
J_{W}^{\{\varepsilon\}}\left(\lambda^{[\varepsilon]}\right):=\mathcal{C}^{\{\varepsilon\}} \cap \tau^{[\varepsilon]}\left(-\lambda^{[\varepsilon]}\right) \mathcal{C}_{+}^{[\varepsilon]} \cap \tau^{[\varepsilon]}\left(\lambda^{[\varepsilon]}\right) \mathcal{C}_{-}^{[\varepsilon]}
$$

Then $J_{W}^{\{\varepsilon\}}\left(\lambda^{[\varepsilon]}\right)$ is an open semi-algebraic polyhedron in $S_{W, \mathbb{R}}^{[\varepsilon]}$ isomorphic to the l-dimensional parallelotope $\left(0, \lambda^{[\varepsilon]}\right)^{l}$ adjacent to the origin o $\in S_{W, \mathbb{C}}$. Let $a o^{\{\varepsilon\}}\left(\lambda^{[\varepsilon]}\right)$ be the vertex of $J_{W}^{\{\varepsilon\}}\left(\lambda^{[\varepsilon]}\right)$ which is antipodal to the origin. Then faces in $\bar{J}_{W}^{\{\varepsilon\}}\left(\lambda^{[\varepsilon]}\right)$ are crossing normally at any point of any closed edge adjacent to $a o^{\{\varepsilon\}}\left(\lambda^{[\varepsilon]}\right)$.

Remark 1. The explicit identification $c_{W}: \bar{J}_{W}^{\{\varepsilon\}}\left(\lambda^{[\varepsilon]}\right) \simeq\left[0,-\lambda^{[\varepsilon]}\right]^{l_{1}} \times$ $\left[0, \lambda^{[\varepsilon]}\right]^{l_{2}}$ with $l=l_{1}+l_{2}$ is given in Theorem $\mathrm{C}$ in $\S 3.5$.

In an assertion in $\S 3.4$, we prove a stronger normal crossing property of faces of $\bar{J}_{W}^{\{\varepsilon\}}\left(\lambda^{[\varepsilon]}\right)$, which implies that the inverse image in $V_{\mathbb{R}}^{\varepsilon}$ of any facet of $\bar{J}_{W}^{\{\varepsilon\}}\left(\lambda^{[\varepsilon]}\right)$ adjacent to $a o^{\{\varepsilon\}}\left(\lambda^{[\varepsilon]}\right)$ is smooth. This gives the next theorem, stated as Theorem B in the introduction.

Theorem B. For $\lambda^{[\varepsilon]} \in \mathbb{R}_{>0}$ and for $\varepsilon \in\{ \pm 1\}$, put

$$
\bar{K}_{W}^{\varepsilon}\left(\lambda^{[\varepsilon]}\right):=\left(\pi_{W, \mathbb{R}}^{\varepsilon}\right)^{-1}\left(\bar{J}_{W}^{\{\varepsilon\}}\left(\lambda^{[\varepsilon]}\right)\right)
$$

Then $\bar{K}_{W}^{\varepsilon}\left(\lambda^{[\varepsilon]}\right)$ is a closed semi-algebraic polyhedron in $V_{\mathbb{R}}^{\varepsilon}$ dual to the simplicial cone decomposition (1.2.2). The $W$-action induces

$$
\bar{K}_{W}^{\varepsilon}\left(\lambda^{[\varepsilon]}\right) / W \cong \pi_{W, \mathbb{R}}^{\varepsilon}\left(\bar{K}_{W}^{\varepsilon}\left(\lambda^{[\varepsilon]}\right)\right)=\bar{J}_{W}^{\{\varepsilon\}}\left(\lambda^{[\varepsilon]}\right) .
$$

Remark 2. The change $D$ to $\omega D$ for $\omega \in \mathbb{R}^{\times}$induces the change $K_{W}^{\varepsilon}$ $\left(\lambda^{[\varepsilon]}, D\right)=\left|\frac{\lambda^{[\varepsilon]}}{\lambda^{[\varepsilon]^{\prime}}} \omega\right|^{1 / h} K_{W}^{\varepsilon}\left(\lambda^{[\varepsilon]^{\prime}}, \omega D\right)$ for $\lambda^{[\varepsilon]}, \lambda^{[\varepsilon]^{\prime}} \in \mathbb{R}_{>0}$, i.e. the polyhedra for any scale $\omega$ and any parameter $\lambda^{[\varepsilon]}$ are homothetic to each other. 


\section{§2. The Central Region $E_{W}^{\{\varepsilon\}}$ in $T_{W, \mathbb{R}}^{[\varepsilon]}$}

The concept of a universal unfolding and its bifurcation set is due to Thom [T2] and is studied by several authors (e.g. [Ar],[Lo1],[Ly],[Te]). We re-introduce the bifurcation set in the setting of $W$ - $\tau$-invariant theory, and then introduce the central region $E_{W}^{\{\varepsilon\}}$, which is a key concept in the present paper. Several results are extracted from [S4]. For a comprehensive study of them with proofs, one is referred to [S4].

\section{§2.1. $\tau$-quotient space $T_{W}$ and $\tau$-quotient morphism $\pi_{\tau}$}

We first introduce the $\tau$-quotient space and the $\tau$-quotient morphism. Recall the co-action $\tau^{*}(1.6 .3)$ and consider the ring of its invariants:

$$
\begin{aligned}
S\left(V_{\mathbb{R}}^{*}\right)^{W, \tau}: & =\left\{f \in S\left(V_{\mathbb{R}}^{*}\right)^{W} \mid \tau^{*}(\lambda) f=f\right\} \\
& =\left\{f \in S\left(V_{\mathbb{R}}^{*}\right)^{W} \mid D f=0\right\} .
\end{aligned}
$$

The associated $\tau$-quotient variety is denoted by

$$
T_{W}:=\operatorname{Spec}\left(S\left(V_{\mathbb{R}}^{*}\right)^{W, \tau}\right) .
$$

One has the $\tau$-quotient morphism

$$
\pi_{\tau}: S_{W} \longrightarrow T_{W}
$$

induced by the inclusion $S\left(V_{\mathbb{R}}^{*}\right)^{W, \tau} \subset S\left(V_{\mathbb{R}}^{*}\right)^{W}$. Using the coordinates $P_{1}, \ldots, P_{l}$, one has the explicit expressions:

$$
S\left(V_{\mathbb{R}}^{*}\right)^{W, \tau}=\mathbb{R}\left[P_{1}, \ldots, P_{l-1}\right] \text { and } S\left(V_{\mathbb{R}}^{*}\right)^{W}=S\left(V_{\mathbb{R}}^{*}\right)^{W, \tau}\left[P_{l}\right] .
$$

Namely, $T_{W}$ is an affine variety with the coordinates $\left(P_{1}, \ldots, P_{l-1}\right)$ and $\pi_{\tau}$ is the projection forgetting the last coordinate $P_{l}$ of $S_{W}$.

\section{$\S 2.2$. Bifurcation divisor $B_{W}=\cup_{p=2}^{\infty} B_{W, p}$}

We introduce the bifurcation set $B_{W}$ in $T_{W}$ as the ramification divisor of the finite cover $\pi_{\tau} \mid D_{W}: D_{W} \rightarrow T_{W}$. Recall that the discriminant $\Delta_{W}$ is a monic polynomial in $P_{l}$ (1.4.4). The resultant of $\Delta_{W}$ and $D \Delta_{W}=\partial_{P_{l}} \Delta_{W}$ with respect to the variable $P_{l}$ is an element in $S\left(V_{\mathbb{R}}^{*}\right)^{W, \tau}$. Decompose it as

$$
\delta\left(\Delta_{W}, D \Delta_{W}\right)=\prod_{p \geq 2} \omega_{W, p}^{p}
$$

according to its multiplicity (=ramification index) $p$, where $\omega_{W, p}$ are multiplicity-free polynomials in $S\left(V_{\mathbb{R}}^{*}\right)^{W, \tau}$. Using the $p$-th factor $\omega_{W, p}$, the $p$-th bifurcation divisor is defined by the equation:

$$
B_{W, p}:=\text { the divisor in } T_{W} \text { defined by } \omega_{W, p}=0 .
$$


We call $B_{W, 2}$ the ordinary part, $B_{W \text {,odd }}:=\cup_{p \text { :odd }} B_{W, p}$ the odd part and $B_{W, \geq 3}:=\cup_{p \geq 3} B_{W, p}$ the higher part of the bifurcation divisor.

Note. The $p$-th bifurcation divisor $B_{W, p}$ is the image of the union of two-codimensional subspaces of $V$ where $p$ reflection hyperplanes are intersecting (see the last formula $(*)$ at the end of Appendix in order to justify the decomposition (2.2.1)).

One basic formula which plays a key role in the sequel is the following:

$$
\operatorname{det}\left(\frac{\partial\left(\Delta_{W}, D \Delta_{W}, \ldots, D^{l-1} \Delta_{W}\right)}{\partial\left(P_{1}, P_{2}, \ldots, P_{l}\right)}\right)=c \cdot \prod_{p \geq 2} \omega_{W, p}^{p-1} .
$$

The proof uses the degree of $\omega_{W, p}$, obtained by the case by case study (see $[\mathrm{S} 4,(3.6 .1)])$.

\section{§2.3. Twisted real forms of the $\tau$-action and the $\tau$-quotient space}

Let $u \in \mathrm{GL}\left(V_{\mathbb{R}}\right)$ be an element of the normalizer $N(W)$ of $W$. We denote by $[u]$ its $W$-coset class in $N(W) / W$. Assume $[u]^{2}=1$ and define an anti- $\mathbb{C}$ linear automorphism $[u]^{a *}: S\left(V_{\mathbb{C}}^{*}\right)^{W} \rightarrow S\left(V_{\mathbb{C}}^{*}\right)^{W}$ by $[u]^{a *} P:=\bar{P} \circ u$. Then the twisted real form $S_{W, \mathbb{R}}^{[u]}:=\operatorname{Hom}_{\mathbb{R}}\left(S\left(V_{\mathbb{C}}^{*}\right)^{W,[u]^{a *}}, \mathbb{R}\right)$ is given by the subalgebra $S\left(V_{\mathbb{C}}^{*}\right)^{W,[u]^{a *}}$ of $[u]^{a *}$-invariants (see [Lo2], [S4]).

Assertion 2.1. $\quad$ There exists $b[u] \in\{ \pm 1\}$ making the following diagram commutative:

$$
\begin{array}{ll}
S\left(V_{\mathbb{C}}^{*}\right)^{W} & \stackrel{\tau^{*}}{\rightarrow} S\left(V_{\mathbb{C}}^{*}\right)^{W} \otimes_{\mathbb{C}} \mathbb{C}[\lambda] \\
\underset{\downarrow}{\mid}[u]^{a *} & \downarrow[u]^{a *} \otimes(b[u] \circ \text { complex conjugation }) \\
S\left(V_{\mathbb{C}}^{*}\right)^{W} & \stackrel{\tau^{*}}{\rightarrow} S\left(V_{\mathbb{C}}^{*}\right)^{W} \otimes_{\mathbb{C}} \mathbb{C}[\lambda] .
\end{array}
$$

One can choose a generator $P_{l}$ satisfying $[u]^{a *} P_{l}=b[u] P_{l}$ so that $P_{l}^{[u]}:=P_{l} / \sqrt{b[u]}$ is a $[u]^{a *}$-invariant. The co-action $\tau^{*}(1.6 .3)$ turns out to be

$$
\tau^{*}: S\left(V_{\mathbb{C}}^{*}\right)^{W,[u]^{a *}} \rightarrow S\left(V_{\mathbb{C}}^{*}\right)^{W,[u]^{a *}} \otimes_{\mathbb{R}} \mathbb{R}[\lambda / \sqrt{b[u]}] .
$$

Accordingly, we introduce a new real variable $\lambda^{[u]}:=\lambda / \sqrt{b[u]}$ so that we obtain a twisted real $\tau$-action

$$
\begin{aligned}
\tau^{[u]}: & \mathbb{R} \times S_{W, \mathbb{R}}^{[u]} \\
\lambda^{[u]} \times\left(P_{1}^{[u]}, \ldots, P_{l}^{[u]}\right) & \mapsto\left(P_{1}^{[u]}, \ldots, P_{l}^{[u]}+\lambda^{[u]}\right) .
\end{aligned}
$$

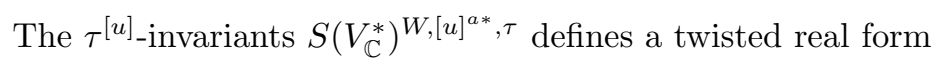

$$
T_{W, \mathbb{R}}^{[u]}:=\operatorname{Hom}_{\mathbb{R}}^{a l g}\left(S\left(V_{\mathbb{C}}^{*}\right)^{W,[u]^{a *}, \tau}, \mathbb{R}\right)
$$


of the space $T_{W, \mathbb{C}}$ together with the twisted real quotient morphism

$$
\pi_{\tau}^{[u]}: S_{W, \mathbb{R}}^{[u]} \longrightarrow T_{W, \mathbb{R}}^{[u]}
$$

One sees from the coordinate expression that the morphism (2.3.5) is an honest set-theoretical quotient map. In the present article, we are interested in the case $[u]=[\varepsilon]$ for $\varepsilon \in\{ \pm 1\}$. In such a case, one has $b[u]=\varepsilon^{h}$.

\section{$\S 2.4$. Subspace $S_{W\left(I_{2}(h)\right)}$ of $S_{W}$}

We introduce a canonical two-dimensional subspace $S_{W\left(I_{2}(h)\right)}$ of $S_{W}$ based on the study of regular eigenvectors of a Coxeter element due to Coleman [C] and Kostant $[\mathrm{K}]$ (cf. [B, Ch.5, §6, no 2, lemma 2]).

Let $C$ be a Weyl chamber in $V_{\mathbb{R}}$ of $W$ and let $\Pi=\Pi(C)$ be the set of attached simple reflections. Recall the Coxeter diagram structure $\Gamma(W)$ on $\Pi$, where one puts an edge between two vertices $\alpha$ and $\beta \Pi$ if $m_{\alpha \beta} \geq 3$. Since $\Gamma(W)$ is a tree, one has a unique decomposition up to a transposition:

$$
\Pi=\Pi_{1} \sqcup \Pi_{2}
$$

where each $\Pi_{j}$ is a totally disconnected subset in $\Gamma(W)$. Put

$$
c_{i}:=\Pi_{\alpha \in \Pi_{i}} \alpha, \quad c:=c_{1} c_{2} \quad \text { and } \quad d:=c_{1}+c_{2},
$$

where $c$ and $d$ are called the Coxeter element and the Killing element.

$c=c_{1} c_{2}$ is of order $h$, and $d$ has only real eigenvalues $2 \cos \left(\pi m_{i} / h\right)$. Let $e$ be an eigenvector of $d$ belonging to the largest eigenvalue $2 \cos (\pi / h)$ (which has multiplicity 1). We can choose $e$ such that its expression with respect to the basis $e_{\alpha}(\alpha \in \Pi)$ (see 1.1, 4.) has all positive real coefficients, so that $e$ is unique up to a positive real constant multiple. Put

$$
U:=\mathbb{R} e_{1}+\mathbb{R} e_{2},
$$

where we use the decomposition $e=e_{1}+e_{2}$ with $e_{j} \in \sum_{\alpha \in \Pi_{j}} \mathbb{R}_{>0} e_{\alpha}$ for $j=1,2$.

Assertion 2.2. $\quad c_{i}$ leaves the space $U$ invariant and $c_{i} \mid U$ is a reflection with respect to $e_{i}$. The restriction homomorphism $\left\langle c_{1}, c_{2}\right\rangle \rightarrow\left\langle c_{1}\left|U, c_{2}\right| U\right\rangle$ is an isomorphism and defines a faithful $W\left(I_{2}(h)\right)$-action on $U$.

Here $W\left(I_{2}(h)\right)$ is the dihedral group of order $2 h$ generated by reflections $c_{1} \mid U$ and $c_{2} \mid U$. Therefore, we introduce

i) the quotient space $S_{W\left(I_{2}(h)\right)}:=U / / W\left(I_{2}(h)\right)$,

ii) a primitive vector field $\frac{\partial}{\partial S}$ (which, we shall define below in $\S 2.5$ ), 
iii) the $\tau$-action on $S_{W\left(I_{2}(h)\right)}$ as the integration of $\frac{\partial}{\partial S}$,

iv) the $\tau$-quotient variety $T_{W\left(I_{2}(h)\right)}:=S_{W\left(I_{2}(h)\right)} / / \tau$.

The embedding $U \subset V_{\mathbb{R}}$ induces the morphism $S_{W\left(I_{2}(h)\right)} \rightarrow S_{W}$. The following facts are not difficult, but need proofs (cf. [S4]).

Assertion 2.3. $\quad$ i) The morphism $S_{W\left(I_{2}(h)\right)} \rightarrow S_{W}$ is a closed embedding. Its image is independent of the choices of a chamber $C$, a decomposition (2.4.1) and an eigenvector $e$.

ii) The primitive vector field on $S_{W}$ is tangent to $S_{W\left(I_{2}(h)\right)}$, and induces a non-zero constant multiple of the primitive vector field on the subspace $S_{W\left(I_{2}(h)\right)}$.

iii) The restriction of the $\tau$-action on $S_{W}$ coincides with the $\tau$-action on $S_{W\left(I_{2}(h)\right)}$ (up to a scaling constant).

iv) One obtains a canonical embedding $T_{W\left(I_{2}(h)\right)} \rightarrow T_{W}$ which makes the following diagram commutative and Cartesian:

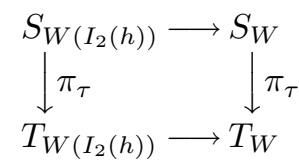

The image of $S_{W\left(I_{2}(h)\right)}$ in $S_{W}$ is called the vertex orbit plane and that of $T_{W\left(I_{2}(h)\right)}$ is called the vertex orbit line. In the sequel, we write v.o. for vertex orbit for short (see the introduction for the naming).

\section{§2.5. V.o. axis, v.o. line and the sign factor $\sigma\left(D,\left\{\Pi_{1}, \Pi_{2}\right\}\right)$}

Depending on a choice of the vector $e$ and the partition $\left\{\Pi_{1}, \Pi_{2}\right\}$, we obtain some particular generators of $S\left[U^{*}\right]^{W\left(I_{2}(h)\right)}$ (called the flat coordinates), which leads to some new concepts and constructions.

First, we identify the vector space $U$ with the complex plane $\mathbb{C}$ regarded as a real vector space $\mathbb{R} \oplus \mathbb{R} i$, where, in order to avoid the confusion with the complex number field as the coefficient field, we use notation $i$ for the unit of pure imaginary number in the plane instead of $\sqrt{-1}$.

i) The identification $U \simeq \mathbb{R} \oplus \mathbb{R} i$ is given by the basis correspondence: $e_{1} \leftrightarrow i$ and $e_{2} \leftrightarrow-i \omega$, where $\omega:=\exp (\pi i / h)=\cos (\pi / h)+\sin (\pi / h) i$.

ii) For $z_{1}, z_{2} \in \mathbb{C} \simeq U$, put $I\left(z_{1}, z_{2}\right)=\operatorname{Re}\left(z_{1} \bar{z}_{2}\right)$.

iii) The generators $c_{1} \mid U$ and $c_{2} \mid U$ of $W\left(I_{2}(h)\right)$ are the reflections with respect to $e_{1}$ and $e_{2}: c_{1}(z)=\bar{z}$ and $c_{2}(z)=\omega^{2} \bar{z}$ on $\mathbb{C}$.

iv) The Coxeter element $c \mid U$ of $W\left(I_{2}(h)\right)$ is identified with the multiplication by $\omega^{-2}$. 
v) We choose the generators $R$ and $S$ of $S\left[U^{*}\right]^{W\left(I_{2}(h)\right)}$ :

$$
\begin{aligned}
& R:=R\left(\left\{\Pi_{1}, \Pi_{2}\right\}, e\right):=z \bar{z}=x^{2}+y^{2}, \\
& S:=S\left(\left\{\Pi_{1}, \Pi_{2}\right\}, e\right):=\operatorname{Re}\left(z^{h}\right)=\sum_{k=0}^{h / 2\rfloor}(-1)^{k} C_{2 k}^{h} x^{h-2 k} y^{2 k} .
\end{aligned}
$$

vi) The changes $e \mapsto r e\left(r \in \mathbb{R}_{>0}\right)$ and $\left\{\Pi_{1}, \Pi_{2}\right\} \mapsto\left\{\Pi_{2}, \Pi_{1}\right\}$ induce:

$$
\begin{aligned}
& R\left(\left\{\Pi_{1}, \Pi_{2}\right\}, r e\right)=r^{2} R\left(\left\{\Pi_{1}, \Pi_{2}\right\}, e\right) \\
& S\left(\left\{\Pi_{1}, \Pi_{2}\right\}, r e\right)=r^{h} S\left(\left\{\Pi_{1}, \Pi_{2}\right\}, e\right) \\
& R\left(\left\{\Pi_{2}, \Pi_{1}\right\}, e\right)=R\left(\left\{\Pi_{1}, \Pi_{2}\right\}, e\right) \\
& S\left(\left\{\Pi_{2}, \Pi_{1}\right\}, e\right)=-S\left(\left\{\Pi_{1}, \Pi_{2}\right\}, e\right) .
\end{aligned}
$$

After the preparation above, we can now clarify several sign problems as follows.

1. Sign factor. The derivation $\frac{\partial}{\partial S}$ is a primitive vector field on $S_{W\left(I_{2}(h)\right)}$. Due to Assertion 2.3, ii), the proportion $\frac{\partial}{\partial S}: D \mid S_{W\left(I_{2}(h)\right)}$ is in $\mathbb{R}^{\times}$. Depending on the choice of the primitive vector field $D(1.6 .1)$ and the decomposition $\left\{\Pi_{1}, \Pi_{2}\right\}$ (2.4.1), we introduce the sign factor:

$$
\sigma\left(D,\left\{\Pi_{1}, \Pi_{2}\right\}\right):=\operatorname{sign}\left(\frac{\partial}{\partial S}: D \mid S_{W\left(I_{2}(h)\right)}\right) \in\{ \pm 1\} .
$$

A typical use of the sign factor is the following [S4,5.1, Sign Theorem]:

Assertion 2.4. For $i \in\{1,2\}$ and $\varepsilon \in\{ \pm 1\}$, define the twisted real vector space with respect to $\varepsilon c_{i}$ by $V_{\mathbb{R}}^{\varepsilon c_{i}}:=\left\{v \in V_{\mathbb{C}} \mid c_{i}(v)=\varepsilon \bar{v}\right\}$. Then $\pi_{W}\left(V_{\mathbb{R}}^{\varepsilon c_{i}}\right)$ is the closure of a connected component of $S_{W, \mathbb{R}}^{[\varepsilon]} \backslash D_{W, \mathbb{R}}^{[\varepsilon]}$ denoted by $\mathcal{C}^{\left\{\varepsilon c_{i}\right\}}$. The components $\mathcal{C}^{\left\{\varepsilon c_{i}\right\}}(i=1,2)$ and the opposite components $\mathcal{C}_{+}^{[\varepsilon]}$ and $\mathcal{C}_{-}^{[\varepsilon]}(1.7 .1)$ are related by the formula:

$$
\mathcal{C}^{\left\{\varepsilon c_{i}\right\}}=\mathcal{C}_{\varepsilon\lfloor h / 2\rfloor}^{[\varepsilon]}(-1)^{i-1} \sigma\left(D,\left\{\Pi_{1}, \Pi_{2}\right\}\right) \text { for } i=1,2 .
$$

The sign factor appears again in Theorem $\mathrm{C}$ in $\S 3.5$.

2. Vertex orbit axis $A O$. Let us call the coordinate axis defined by $S=0$ in the v.o. plane $S_{W\left(I_{2}(h)\right)}$ the vertex orbit axis. It is a one-dimensional line in $S_{W}$. Note the fact that the coordinate $R$ (c.f. (2.5.1)) is unique up to a positive constant multiple. Thus, the real v.o. axis and the half v.o. axis

$$
\begin{aligned}
A O & :=\left\{(R, S) \in S_{W\left(I_{2}(h)\right), \mathbb{C}} \mid R \in \mathbb{R} \text { and } S=0\right\} \\
A O^{ \pm} & :=\left\{(R, S) \in S_{W\left(I_{2}(h)\right), \mathbb{C}} \mid R \in \pm \mathbb{R}_{>0} \text { and } S=0\right\}
\end{aligned}
$$

are a real line and real half lines in $S_{W, \mathbb{C}}$ well defined independent of the choices of a chamber $C$, a partition $\left\{\Pi_{1}, \Pi_{2}\right\}$ or a vector $e$. 
Assertion 2.5. $\quad$ i) For any $\varepsilon \in\{ \pm 1\}$, the twisted real form $S_{W, \mathbb{R}}^{[\varepsilon]}$ contains the full real v.o. axis $A O$ (see Remark 3. below).

ii) For $\varepsilon \in\{ \pm 1\}$, the connected component of $S_{W, \mathbb{R}}^{[\varepsilon]} \backslash D_{W, \mathbb{R}}^{[\varepsilon]}$ containing the half v.o. axis $A O^{\varepsilon}$ is the central component $\mathcal{C}^{\varepsilon}$ (recall (1.5.2)).

In $\S 4.1$ of the present article, we shall use $A O^{+}$and $A O^{-}$as the base point locus for the fundamental group of $S_{W, \mathbb{C}} \backslash D_{W, \mathbb{C}}$.

3. Vertex orbit line $O$. Recall the one-dimensional subspace $T_{W\left(I_{2}(h)\right)}$ of $T_{W}$ (called the v.o. line) which is the projection image of the vertex orbit axis by $\pi_{\tau}$ (c.f. (2.4.4)). Similarly to the case of the vertex orbit axis, its coordinate $R$ is unique up to a positive constant multiple. Thus, the real v.o. line and the half v.o. lines are given by

$$
\begin{gathered}
O:=\left\{(R) \in T_{W\left(I_{2}(h)\right)} \mid R \in \mathbb{R}\right\}, \\
O^{ \pm}:=\left\{(R) \in T_{W\left(I_{2}(h)\right)} \mid R \in \pm \mathbb{R}_{>0}\right\} .
\end{gathered}
$$

They are a well-defined real line and real half lines in $T_{W, \mathbb{C}}$.

Assertion 2.6. $\quad$ i) For any $\varepsilon \in\{ \pm 1\}$, the twisted real form $T_{W, \mathbb{R}}^{[\varepsilon]}$ contains the real v.o. line $O$ (see Remark 3 . below).

ii) For $\varepsilon \in\{ \pm 1\}$, the half v.o. line $O^{\varepsilon}$ does not intersect the higher bifurcation locus $B_{W, \geq 3, \mathbb{C}}$ in $T_{W, \mathbb{C}}$.

Due to this assertion, we are able to deine:

Definition. We introduce

$$
\begin{aligned}
E_{W}^{\{\varepsilon\}}:= & \text { the connected component of } T_{W, \mathbb{R}}^{[\varepsilon]} \backslash B_{W, \geq 3, \mathbb{R}}^{[\varepsilon]} \\
& \text { containing the half v.o. line } O^{\varepsilon}
\end{aligned}
$$

and call it the central region in $T_{W, \mathbb{R}}^{[\varepsilon]}$.

Remark 3. The statements i) in Assertion 2.5 and i) in 2.6 are valid for any twisted real structures $[u]$ on $S_{W}$ and $T_{W}$, respectively. The connected component $E_{W}^{[u], \varepsilon}$ of $T_{W, \mathbb{R}}^{[u]} \backslash B_{W, \geq 3, \mathbb{R}}^{[u]}$ containing $O^{\varepsilon}$ are studied in [S4,§10].

Remark 4. Actually, the v.o. line $O$ is contained in the ordinary bifurcation set $B_{W, 2}$ for $l \geq 3$. Hence, the ordinary bifurcation set intersects the central region for $l \geq 3$. The description of the intersection $E_{W}^{\{\varepsilon\}} \cap B_{W, 2, \mathbb{R}}^{[\varepsilon]}$ is reduced to certain real linear inequalities by use of Theorem $\mathrm{C}$ in $\S 3.5$. It proposes a quite interesting and important combinatorial geometric problem. 


\section{§2.6. Algebroid functions $\varphi_{\alpha}$ and $\phi_{\alpha}$ for $\alpha \in \Pi$}

Since we have fixed the base point locus, either $O^{+}$or $O^{-}$, in $T_{W, \mathbb{C}}$, we are able to discuss multi-valued functions and, in particular, algebroid functions defined on $T_{W, \mathbb{C}} \backslash B_{W, \geq 3, \mathbb{C}}$.

Recall the discriminant (1.4.4). It is a monic polynomial of degree $l$ in the indeterminate $P_{l}$. Let us regard it as the equation in $P_{l}$ :

$$
\Delta_{W}=A_{0} P_{l}^{l}+A_{1} P_{l}^{l-1}+\cdots+A_{l-1} P_{l}+A_{l}=0,
$$

where the coefficients $A_{i}$ are polynomial functions on $T_{W, \mathbb{C}}$. One can show that i) for any point of $T_{W, \mathbb{C}} \backslash B_{W \text {,odd, }}$, there exist a neighborhood $\mathcal{V}$ and $l$ holomorphic functions $f_{1}, \ldots, f_{l}$ on $\mathcal{V}$ such that $f_{1}(t), \ldots, f_{l}(t)$ are the solutions of the equation (2.6.1) at $t \in \mathcal{V}$, and ii) the functions $f_{1}, \ldots, f_{l}$ can be analytically continued to everywhere in $T_{W, \mathbb{C}} \backslash B_{W \text {,odd, } \mathbb{C}}$.

As for an initial system, let us choose $l$ functions indexed by $\alpha \in \Pi$ :

$$
\varphi_{\alpha, \varepsilon}
$$

on a neighborhood of the base point locus $O^{\varepsilon}$ as follows (see $[\mathrm{S} 4, \S 8,9]$ ).

For $\alpha \in \Pi_{i}$, let $\zeta_{i} \in U(i=1,2)$ be a point $(\neq 0)$ fixed by the action of $c_{i} \mid U$ on the real 2-space $U$. Since $c_{i} \mid U$ is a reflection with respect to $e_{i}$ (recall Assertion 2.2), we see $e_{i} \cdot \zeta_{i}=0$, where $x \cdot y$ is a $W\left(I_{2}(h)\right)$-invariant positive symmetric bilinear form on $U$. Taking the fact $e_{1} \cdot e_{2}<0$ into account, the coefficients $a, b \in \mathbb{R}$ in the expression $\zeta_{i}=a e_{1}+b e_{2}$ are simultaneously positive or negative. We choose $\zeta_{i}$ such that $a, b>0$. Then $\zeta_{i}$ is unique up to a positive constant multiple (see Remark 6).

For $\varepsilon \in\{ \pm 1\}$, put $\zeta_{i}^{\varepsilon}:=\sqrt{\varepsilon} \zeta_{i} \in U^{\varepsilon}:=\sqrt{\varepsilon} \otimes U$ (see Remark 7). It projects by $\pi_{W}$ to a point $p_{i}$ on the discriminant locus $D_{W\left(I_{2}(h)\right), \mathbb{R}}^{[\varepsilon]} \subset S_{W\left(I_{2}(h)\right), \mathbb{R}}^{[\varepsilon]}$, and $p_{i}$ projects further to a point $q_{i} \in O^{\varepsilon} \subset T_{W, \mathbb{R}}^{[\varepsilon]}$ by $\pi_{\tau}$ (c.f. (2.4.4)). On the other hand, the reflection hyperplane $H_{\alpha}$ for $\alpha \in \Pi_{i}$ contains $\zeta_{i}$. Hence, $\zeta_{i}^{\varepsilon} \in H_{\alpha, \mathbb{C}}$. Since $H_{\alpha}\left(\alpha \in \Pi_{i}\right)$ are normally crossing at $\zeta_{i}$, the stabilizer subgroup $W\left(\zeta_{i}\right)$ of $\zeta_{i}$ is an abelian group $\left\langle\beta, \beta \in \Pi_{i}\right\rangle \simeq\left(\mathbb{Z}_{2}\right)^{\# \Pi_{i}}$ and preserves each hyperplane $H_{\alpha}$ for $\alpha \in \Pi$. Then, it is easy to see that a neighborhood of $\zeta_{i}^{\varepsilon}$ in $H_{\alpha, \mathbb{C}}$ projects (by $2^{\# \Pi_{i}-1}$ to one) onto a neighborhood $\mathcal{U}_{\alpha}$ of $p_{i}$ in one of $\# \Pi_{i}$-number of local irreducible components of $D_{W, \mathbb{C}}$ at $p_{i}$. We can show that $q_{i}=\pi_{\tau}\left(p_{i}\right) \notin B_{W, \text { odd }, \mathbb{C}}$ and that the projection $\pi_{\tau} \mid \mathcal{U}_{\alpha}$ is a locally homeomorphism onto a neighborhood $\mathcal{V}_{\alpha}$ of $q_{i}$ in $T_{W, \mathbb{C}}$. Then, we reverse the $\operatorname{map} \pi_{\tau} \mid \mathcal{U}_{\alpha}$ to a map $\varpi_{\alpha}:\left(\mathcal{V}_{\alpha}, q_{i}\right) \rightarrow\left(\mathcal{U}_{\alpha}, p_{i}\right)$, and put

$$
\varphi_{\alpha, \varepsilon}:=P_{l} \circ \varpi_{\alpha} .
$$


By definition, $\varphi_{\alpha, \varepsilon}$ is a solution to the discriminant equation (2.6.1) on a neighborhood of $O^{\varepsilon}$. Thus we obtain the system of algebroid functions indexed by $\Pi$ (2.6.2). By use of characteristic variety $C_{W}$, we observe that these give the full system of solutions of the discriminant equation. That is: one has the "local factorization" of the discriminant:

$$
\Delta_{W}=A_{0} \prod_{\alpha \in \Pi}\left(P_{l}-\varphi_{\alpha, \varepsilon}\right),
$$

on a neighborhood of the base point locus $O^{\varepsilon} \subset T_{W, \mathbb{C}}$. We set

$$
\phi_{\alpha, \varepsilon}:=P_{l}-\varphi_{\alpha, \varepsilon} \quad \text { for } \quad \alpha \in \Pi .
$$

Since $D^{i} \Delta_{W}$ is, up to the factor $A_{0}$, equal to the $(l-i)$-th elementary symmetric

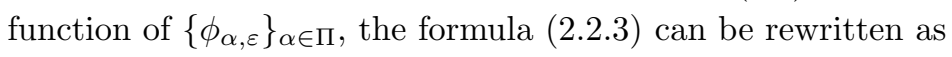

$$
\wedge_{\alpha \in \Pi} d \phi_{\alpha, \varepsilon}=c \prod_{p \geq 3} \omega_{W, p}^{p / 2-1} \cdot \wedge_{i=1}^{l} d P_{i} \quad \text { for some } c \in \mathbb{R}^{\times} .
$$

Remark 5. In the next $\S 3.4$, we introduce a largest covering space of $T_{W, \mathbb{C}} \backslash \mathbb{B}_{W \text {,odd, } \mathbb{C}}$ with liftings of the base point loci $O^{+}$and $O^{-}$such that $\varphi_{\alpha,+}$ and $\varphi_{\alpha,-}$ are lifted to functions defined on the neighborhoods of the base point loci and are analytically continued to the same univalent function.

Remark 6. In the above construction of $\varphi_{\alpha, \varepsilon}$, we have chosen $\zeta_{i}$ in such a manner that the coefficients with respect to the basis $e_{i}$ are positive. However, we may choose $-\zeta_{i}$ as the starting point of the construction. Then, for $\varepsilon \in$ $\{ \pm 1\}$, the function $\varphi_{\alpha, \varepsilon}$ changes to $\varphi_{\chi_{W}(\alpha), \varepsilon}$, where $\chi_{W}$ is the bijection of $\Pi$ induced by the adjoint action of the longest element of $W$ (see [S4,8.11], c.f. also (3.3.1)). This change is caused by the change of the "reference" chamber from $C$ to $-C$, which covers the central component $\mathcal{C}$.

Remark 7. In the above construction of $\varphi_{\alpha, \varepsilon}$, we have chosen $\zeta_{i}^{\varepsilon} \in H_{\alpha}^{\varepsilon}$ to be $\zeta_{i} \sqrt{\varepsilon}$. However, we may choose its complex conjugate $\zeta_{i} / \sqrt{\varepsilon}$ for $\zeta_{i}^{\varepsilon}$ as the starting point of the construction. Then the $\varphi_{\alpha,+1}$ is unchanged, but the $\varphi_{\alpha,-1}$ changes to $\varphi_{\chi_{W}(\alpha),-1}$ (see Remark 5 for notation and reference). This change is caused by the change of the sign of the unit $\sqrt{-1}$ of the pure imaginary number.

\section{§3. Linearization Map $c_{W}$}

The system of algebroid functions $\phi_{\alpha, \varepsilon}$ and $\varphi_{\alpha, \varepsilon}$ introduced in the previous section define, by analytic continuation, multivalued holomorphic maps:

$$
c_{W}=\left(\phi_{\alpha}\right)_{\alpha \in \Pi}: S_{W, \mathbb{C}} \rightarrow \mathbb{C}^{\Pi} \quad \& \quad b_{W}=\left(-\varphi_{\alpha}\right)_{\alpha \in \Pi}: T_{W, \mathbb{C}} \rightarrow \mathbb{C}^{\Pi} / \mathbb{C} v_{\Pi}
$$


where i) $v_{\Pi}=\left(1_{\alpha}\right)_{\alpha \in \Pi}$ is the diagonal element, and ii) " $-\rightarrow$ " means that the "maps" are not univalent on $S_{W, \mathbb{C}}$ or on $T_{W, \mathbb{C}}$ but are defined on their suitable (branched) covering spaces. In order to clarify this multivaluedness, there may be two approaches.

1. Transcendental method: introduce a topological covering space $T_{W, \text { odd, } \mathbb{C}}$ of $T_{W, \mathbb{C}} \backslash B_{W \text {,odd, } \mathbb{C}}$ and lift $b_{W}$ to a univalent holomorphic map $b_{W \text {,odd }}$ defined on it, and similarly for $c_{W \text {,odd }}$.

2. Algebraic method: introduce a suitable finite covering variety $\tilde{T}_{W} \rightarrow T_{W}$ (branching along $B_{W \text {,odd }}$ ) and introduce $\tilde{b}_{W}$ as a scheme morphism from $\tilde{T}_{W}$ to the affine space $V_{\Pi}$, and similarly for $\tilde{c}_{W}$.

The first approach is naive and easily understandable. However, there is a disadvantage that the "boundary points $B_{W \text {,odd }}$ is excluded from the domain of definition. In the second approach those boundary points are naturally included in the domain of definition. Furthermore, it has another advantage that we can discuss about the twisted real forms of the maps (which plays a basic role in our study). For these reasons, we employ the second approach in $[\mathrm{S} 4, \S 10]$. However, the second approach is technically more involved, and we use the first approach to formulate Theorem $\mathrm{C}$ in $\S 3.5$ in the present paper.

An important role of the maps $c_{W}$ and $b_{W}$ is that they identify certain area in $S_{W, \mathbb{C}}$ and in $T_{W, \mathbb{C}}$ with certain area in a linear space $\widehat{V}_{\Pi, \mathbb{C}}$ and in $V_{\Pi, \mathbb{C}}$, respectively. In particular, the map $c_{W}$ identifies the twisted real discriminant locus in a tube domain of the source space with a system of real hyperplanes in a tube domain of the target space. This has several fruitful consequences, since the study of configurations among branches of the real discriminant locus is reduced to a study of a certain system of linear inequalities. By this reason, we call these maps $c_{W}$ and $b_{W}$ the linearization maps.

Let us explain the contents of this section.

The linear model spaces $\widehat{V}_{\Pi}$ and $V_{\Pi}$, which will be the target spaces of the linearization maps, are described in $\S 3.1$. Depending only on the Coxeter graph $\Gamma(W)$, we introduce a simplicial cone $E_{\Gamma(W)}$ in $V_{\Pi, \mathbb{R}}$ in $\S 3.2$. In $\S 3.3$, we introduce the covering space $T_{W \text {,odd, } \mathbb{C}}$, on which the two algebroid functions $\varphi_{\alpha, \varepsilon}$ for $\varepsilon \in\{ \pm 1\}$ in $\S 2.6$ lift to the same globally defined univalent function, denoted by $\varphi_{\alpha}$. By the use of them in $\S 3.4$, the linearization maps $c_{W}$ and $b_{W}$ are defined. In $\S 3.5$, we formulate Theorem $\mathrm{C}$, which states about the comparison of the real spaces $S_{W, \mathbb{R}}^{[\varepsilon]}$ and $\widehat{V}_{\Pi, \mathbb{R}}$ obtained by the linearlization maps. As applications of Theorem C, Theorems A and B are proved in $\S 3.6$ and $\S 3.7$. We illustrate in $\S 3.8$ the linearization maps for the type $A_{3}$. 


\section{§3.1. Linear model spaces $\widehat{V_{\Pi}}$ and $V_{\Pi}$}

We introduce two linear model spaces $\widehat{V_{\Pi}}$ and $V_{\Pi}$, which will be the target spaces of the linearization maps $c_{W}$ and $b_{W}$, respectively.

Define the real vector space with the basis $\left\{v_{\alpha}\right\}_{\alpha \in \Pi}$ :

$$
\widehat{V_{\Pi}}:=\oplus_{\alpha \in \Pi} \mathbb{R} v_{\alpha}
$$

Translation by constant multiples of the diagonal element:

$$
v_{\Pi}:=\sum_{\alpha \in \Pi} v_{\alpha}
$$

defines a $\mathbb{G}_{a}$-action on $\widehat{V_{\Pi}}$ :

$$
(\lambda, \tilde{v}) \in \mathbb{G}_{a} \times \widehat{V_{\Pi}} \mapsto \tilde{v}+\lambda \cdot v_{\Pi} \in \widehat{V_{\Pi}}
$$

The quotient space $V_{\Pi}$ and the quotient map $\pi_{\Pi}$ are introduced by

$$
\pi_{\Pi}: \widehat{V_{\Pi}} \longrightarrow V_{\Pi}:=\widehat{V_{\Pi}} / \mathbb{R} \cdot v_{\Pi} \cdot
$$

The symmetric group $\mathfrak{S}(\Pi)$ acts linearly on $\widehat{V_{\Pi}}$ by permuting the basis $v_{\alpha}$. Since $v_{\Pi}$ is fixed by $\mathfrak{S}(\Pi)$, it induces an action of $\mathfrak{S}(\Pi)$ on the quotient $V_{\Pi}$.

Let $\left\{\lambda_{\alpha}\right\}_{\alpha \in \Pi}$ be the dual basis of $\left\{v_{\alpha}\right\}_{\alpha \in \Pi}$, i.e., the coordinate system of $\widehat{V_{\Pi}}$. The infinitesimal action of the $\mathbb{G}_{a}$-action (3.1.4) is given by

$$
\sum_{\alpha \in \Pi} \frac{\partial}{\partial \lambda_{\alpha}}
$$

Consider the coordinate hyperplane in $\widehat{V_{\Pi}}$ :

$$
H_{\alpha}:=\left\{\sum_{\beta \in \Pi} \lambda_{\beta} v_{\beta} \in \widehat{V_{\Pi}} \mid \lambda_{\alpha}=0\right\}
$$

for $\alpha \in \Pi$. The projection $\pi_{\Pi}$ induces an isomorphism from $H_{\alpha}$ to $V_{\Pi}$ for each $\alpha \in \Pi$, and also an isomorphism from the intersections $H_{\alpha} \cap H_{\beta}$ for $\alpha, \beta \in \Pi$ $(\alpha \neq \beta)$ to the hyperplane in $V_{\Pi}$ :

$$
H_{\alpha \beta}:=\left\{v \in V_{\Pi} \mid \lambda_{\alpha \beta}(v)=0\right\} .
$$

Here $\lambda_{\alpha \beta}:=\lambda_{\alpha}-\lambda_{\beta}$ is a linear form on $V_{\Pi}$, which satisfies

$$
\lambda_{\alpha \beta}+\lambda_{\beta \gamma}=\lambda_{\alpha \gamma} \quad \text { for } \alpha, \beta, \gamma \in \Pi \text {. }
$$

Note. The set of linear forms $\left\{\lambda_{\alpha \beta} \mid \alpha, \beta \in \Pi, \alpha \neq \beta\right\}$ on $V_{\Pi}$ forms a root system of type $A_{l-1}$, where $H_{\alpha \beta}$ are the reflection hyperplanes of the group $\mathfrak{S}(\Pi)$. What is different from the usual setting is the fact that the reflection hyperplane $H_{\alpha \beta}$ is labeled by the positive integer $\operatorname{ord}(\alpha \beta)$. 


\section{§3.2. $\quad \Gamma(W)$-cone $E_{\Gamma(W)}$ in $V_{\Pi}$}

Depending on the graph $\Gamma(W)$ (see $\S 2.4)$ and on the partition $\left\{\Pi_{1}, \Pi_{2}\right\}$ (2.4.1), we introduce an open simplicial cone $E_{\Gamma(W)}$ in $V_{\Pi}$. Put

$$
\begin{aligned}
E_{\Gamma(W):}:= & \text { the connected component of } V_{\Pi} \backslash\left\{\Omega_{\Gamma(W)}=0\right\} \\
& \text { containing the half line } \mathbb{R}_{>0}\left(v_{\Pi_{1}}-v_{\Pi_{2}}\right)
\end{aligned}
$$

where $\Omega_{\Gamma(W)}:=\prod_{\overline{\alpha \beta} \in \operatorname{Edge}(\Gamma(W))} \lambda_{\alpha \beta}^{2}$ and $v_{\Pi_{i}}:=\sum_{\alpha \in \Pi_{i}} v_{\alpha}(i=1,2)$. We call $E_{\Gamma(W)}$ the $\Gamma(W)$-cone. As an immediate consequence of the definition, we have:

Assertion 3.1. The $\Gamma(W)$-cone is given by inequalities:

$$
\begin{aligned}
E_{\Gamma(W)}= & \left\{v \in V_{\Pi} \mid \lambda_{\alpha \beta}(v)>0\right. \\
& \text { for } \left.\alpha \in \Pi_{1}, \beta \in \Pi_{2} \text { such that } \overline{\alpha \beta} \in \operatorname{Edge}(\Gamma(W))\right\} .
\end{aligned}
$$

Therefore, $E_{\Gamma(W)}$ is an open simplicial cone.

The transposition of $\Pi_{1}$ and $\Pi_{2}$ induces the change of the $\Gamma(W)$-cone $E_{\Gamma(W)}$ to $-E_{\Gamma(W)}$. This dependence of the $\Gamma(W)$-cone on the partition of $\Pi$ is important. However, for the sake of simplicity, we omit $\left\{\Pi_{1}, \Pi_{2}\right\}$ in the notation $E_{\Gamma(W)}$ unless explicitly mentioned.

\section{§3.3. Covering spaces $T_{W, \text { odd, } \mathbb{C}}$ and $S_{W, \text { odd, } \mathbb{C}}$}

We introduce a covering space $T_{W \text {,odd, } \mathbb{C}}$ of $T_{W, \mathbb{C}} \backslash B_{W \text {,odd, } \mathbb{C}}$, where we lift the two base point loci $O^{\varepsilon}$ for $\varepsilon \in\{ \pm 1\}$. It turns out that two germs of algebroid functions $\varphi_{\alpha,+1}$ and $\varphi_{\alpha,-1}$, lifted in the neighborhoods of the lifted base point loci, are analytically continuated to the same univalent function, which we shall denote by $\varphi_{\alpha}$, on $T_{W \text {,odd, }}$.

Consider the complexified vertex orbit line $T_{W\left(I_{2}(h)\right), \mathbb{C}}$ (recall $\S 2.4$ and

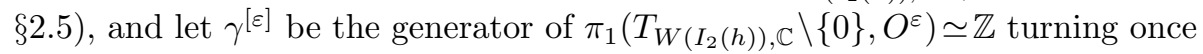
around the origin counter-clockwise. Then one has ([S4,9.2]):

i) $\left(\gamma^{[\varepsilon]}\right)^{2}$ belongs to the center of $\pi_{1}\left(T_{W, \mathbb{C}} \backslash B_{W, \text { odd, } \mathbb{C}}, O^{\varepsilon}\right)$.

ii) the monodromy action of $\gamma^{[\varepsilon]}$ on $\left\{\varphi_{\alpha, \varepsilon}\right\}_{\alpha \in \Pi}(2.6 .2)$ is given by

$$
\varphi_{\alpha, \varepsilon}\left(\tilde{t} \cdot \gamma^{[\varepsilon]}\right)=\varphi_{\chi_{W}(\alpha), \varepsilon}(\tilde{t}),
$$

where $\chi_{W} \in \mathfrak{S}(\Pi)$ is the involution of the set $\Pi$ obtained by the adjoint action of the longest element of $W$.

The fundamental group $\pi_{1}\left(T_{W, \mathbb{C}} \backslash B_{W, \text { odd, } \mathbb{C}}, O^{\varepsilon}\right)$ acts on the universal covering space $\left(T_{W, \mathbb{C}} \backslash B_{W, \text { odd, }}\right)^{r}$ by choosing a base point locus $\tilde{O}^{\varepsilon}$ (i.e., a closed 
set in the covering which projects homeomorphically onto $O^{\varepsilon}$ ). Depending on $\varepsilon \in\{ \pm 1\}$, let us introduce the central quotient space

$$
T_{W, \text { odd }, \mathbb{C}}:=\left(T_{W, \mathbb{C}} \backslash B_{W, \text { odd }, \mathbb{C}}\right)^{r} /\left\langle\left(\gamma^{[\varepsilon]}\right)^{2}\right\rangle
$$

with the base point locus $O_{\text {odd }}^{\varepsilon}:=$ the image of $\tilde{O}^{\varepsilon}$. One has the natural covering

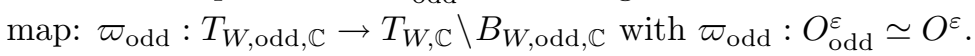

Remark 8. The $T_{W, \text { odd, } \mathbb{C}}$ contains pull-backs of evenly labeled bifurcation set $B_{W, \text { even, } \mathbb{C}} \backslash B_{W, \text { odd, } \mathbb{C}}$ and, in particular, the ordinary part $B_{W, 2, \mathbb{C}}$.

We have constructed two covering spaces depending on $\varepsilon \in\{ \pm 1\}$. In the following, we identify them and consider only one space, denoted again by $T_{W \text {,odd, } \mathbb{C}}$, by choosing the two base point locus $O_{\text {odd }}^{\varepsilon}(\varepsilon \in\{ \pm 1\})$ simultaneously as follows: The inverse image by $\varpi_{\text {odd }}$ of the complexified v.o. line $T_{W\left(I_{2}(h)\right) \text {, }}$ decomposes into the connected components, each of which is a double cover of the complex v.o. line and is isomorphic to $\mathbb{C}^{\times}$and admits a natural $\mathbb{C}^{\times}$-action. Choose one component and fix the base point locus inside it as follows:

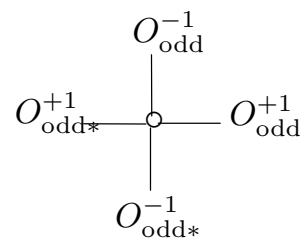

$$
\begin{aligned}
O_{\text {odd }}^{-1} & :=e^{\frac{\pi}{2} \sqrt{-1}} O_{\text {odd }}^{+1} \\
O_{\text {odd } *}^{-1} & :=e^{-\frac{\pi}{2} \sqrt{-1}} O_{\text {odd }}^{+1} \\
O_{\text {odd } *}^{+1} & :=e^{\pi \sqrt{-1}} O_{\text {odd }}^{+1}=e^{-\pi \sqrt{-1}} O_{\text {odd }}^{+1}
\end{aligned}
$$

Figure 1. Four base point loci in $T_{W \text {,odd, } \mathbb{C}}$

where $O_{\text {odd* }}^{\varepsilon}(\varepsilon \in\{ \pm 1\})$ are some auxiliary base point loci (see Remark 9).

The germ of an algebroid function $\varphi_{\alpha, \varepsilon}(2.6 .2)$ is lifted to a germ of holomorphic function, again denoted by $\varphi_{\alpha, \varepsilon}$, on a neighborhood of $O_{\text {odd }}^{\varepsilon}$. We observe that:

i) the germ $\varphi_{\alpha, \varepsilon}$ for $\alpha \in \Pi$ and $\varepsilon \in\{ \pm 1\}$ is analytically continued to a unique univalent holomorphic function on $T_{W, \text { odd, } \mathbb{C}}$,

ii) for each $\alpha \in \Pi$, the two univalent functions defined in i) for $\varepsilon \in\{ \pm 1\}$ define the same function on $T_{W, \text { odd, }}$. Let us denote it by 
Remark 9. The fact i) is an immediate consequence of (3.3.1). The fact ii) is based on the choices of the base point loci in Fig. 1 and of the sign of $\zeta_{i}^{\varepsilon}$ in the construction of $\varphi_{\alpha, \varepsilon}$ in $\S 2.6$. If we choose $\zeta_{i}^{\varepsilon}$ to be the complex conjugate $\zeta_{i} / \sqrt{\varepsilon}$ of $\zeta_{i} \sqrt{\varepsilon}$ for $\varepsilon=-1$, we have to take $O_{\text {odd } *}^{-1}$ instead of $O_{\text {odd }}^{-1}$ as the base point locus. That is: the generator of the Galois group $\operatorname{Gal}(\mathbb{C} / \mathbb{R}) \simeq \mathbb{Z} / 2 \mathbb{Z}$ acts on the index set $\Pi$ of $\left\{\varphi_{\alpha,-1}\right\}_{\alpha \in \Pi}$ by the formula (3.3.1) (see [S4,8.4 \& (9.3.8)]).

Let us introduce the fiber product space: $S_{W \text {,odd, } \mathbb{C}}:=S_{W, \mathbb{C}} \times_{T_{W, \mathbb{C}}} T_{W \text {,odd, } \mathbb{C}}$. On $S_{W, \text { odd, }}$, we introduce a system of univalent holomorphic functions:

$$
\phi_{\alpha}:=P_{l}-\varphi_{\alpha} \text { for } \alpha \in \Pi \text {. }
$$

\section{§3.4. Linearization morphism $c_{W}$ on $S_{W, \text { odd, } \mathbb{C}}$}

We introduce the linearization map $b_{W}$ as the map from the covering space $T_{W, \text { odd, } \mathbb{C}}$ of $T_{W, \mathbb{C}}$ to the complexified model vector spaces $V_{\Pi, \mathbb{C}}$, and similarly $c_{W}$ from $S_{W, \text { odd }, \mathbb{C}}$ to $\widehat{V_{\Pi, \mathbb{C}}}$.

Definition. Using the functions $\varphi_{\alpha}$ (3.3.3) and $\phi_{\alpha}$ (3.3.4) as for the coefficients of $v_{\alpha}$, we consider the maps:

$$
\begin{aligned}
& c_{W, \text { odd }, \mathbb{C}}=\omega^{-1} \sum_{\alpha \in \Pi} \phi_{\alpha} v_{\alpha}: S_{W, \text { odd }, \mathbb{C}} \times \mathbb{C}^{\times} \longrightarrow \widehat{V_{\Pi, \mathbb{C}}}:=\widehat{V_{\Pi}} \otimes_{\mathbb{R}} \mathbb{C} \\
& b_{W, \text { odd, } \mathbb{C}}=-\omega^{-1} \sum_{\alpha \in \Pi} \varphi_{\alpha} v_{\alpha}: T_{W, \text { odd }, \mathbb{C}} \times \mathbb{C}^{\times} \longrightarrow V_{\Pi, \mathbb{C}}:=V_{\Pi} \otimes_{\mathbb{R}} \mathbb{C}
\end{aligned}
$$

and call it the linearization map, where $\omega \in \mathbb{C}^{\times}$is a scaling factor which shall take a special value depending on a choice of a twisted real structure.

The push-forward of the primitive vector field $D$ is given by

$$
\left(c_{W}\right)_{*}(D)=\omega^{-1} \sum_{\alpha \in \Pi} \frac{\partial}{\partial \lambda_{\alpha}} .
$$

namely, $c_{W, \mathbb{C}}$ is equivariant with respect to the two $\mathbb{G}_{a}$-actions: the $\tau$-action on $S_{W}$ and the diagonal translation on $\widehat{V_{\Pi}}$ (multiplied by $\omega$ ). One has

$$
c_{W, \text { odd }, \mathbb{C}}(\tau(\lambda) z, \omega)=c_{W, \text { odd }, \mathbb{C}}(z, \omega)+\omega^{-1} \lambda \cdot v_{\Pi},
$$

and, hence, the following diagram is commutative:

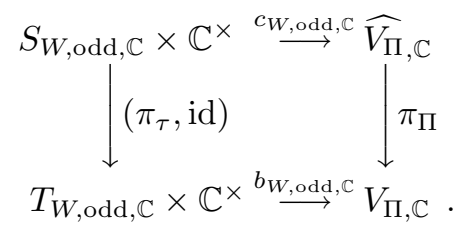


The formula (2.6.5) can be reformulated as the Jacobian formula:

$$
\begin{aligned}
& J a c\left(b_{W, \text { odd }, \mathbb{C}}\right)=c \cdot \omega^{-l} \Pi_{p \geq 3} \omega_{W, p}^{p / 2-1}, \\
& \operatorname{Jac}\left(c_{W, \text { odd }, \mathbb{C}}\right)=c \cdot \omega^{1-l} \pi_{\tau}^{*}\left(\Pi_{p \geq 3} \omega_{W, p}^{p / 2-1}\right) .
\end{aligned}
$$

for some constants $c \in \mathbb{R}^{\times}$. We observe that the factor $\omega_{W, 2}$ does not appear in the right hand side. Therefore,

Assertion 3.2. The holomorphic maps $c_{W, \text { odd, } \mathbb{C}}$ and $b_{W, \text { odd, } \mathbb{C}}$ are not ramifying along the ordinary bifurcation set $\left(\pi_{\tau}\right)^{-1}\left(B_{W, 2, \mathbb{C}}\right)$ and $B_{W, 2, \mathbb{C}}$, but are ramifying along $\left(\pi_{\tau}\right)^{-1}\left(B_{W, 2 p, \mathbb{C}}\right)$ and $B_{W, 2 p, \mathbb{C}}$ for $p \geq 2$, respectively.

\section{§3.5. Theorem C}

In the previous paragraph, the linearization maps are introduced as holomorphic maps from the complex manifolds $T_{W \text {,odd, } \mathbb{C}}$ and $S_{W \text {,odd,C }}$ to the linear model spaces. In this paragraph, we restrict the domain of the definitions of the linearlization maps $b_{W, \text { odd, } \mathbb{C}}$ and $c_{W \text {,odd, } \mathbb{C}}$ to the central region $E_{W}^{\{\varepsilon\}}$ introduced in (2.5.8) and the tube domain $\left(\pi_{\tau}^{[\varepsilon]}\right)^{-1}\left(E_{W}^{\{\varepsilon\}}\right)$ over the central region with a fixed scaling parameter $\omega \in\left\{ \pm \sqrt{\varepsilon^{h}}\right\}$ (see Remark 11 below). The linearization maps for a fixed $\omega$ are denoted by $b_{W, \omega D}$ and $c_{W, \omega D}$, respectively:

$$
b_{W, \omega D}(x):=b_{W, \text { odd }, \mathbb{C}}(x, \omega) \quad \text { and } \quad c_{W, \omega D}(x):=c_{W, \text { odd }, \mathbb{C}}(x, \omega) .
$$

It is a straightforward calculation to observe that for these choices, the image of the linearization maps are contained in the real forms $V_{\Pi}$ and $\widehat{V}_{\Pi}$ (see Remarks 10). Thus, (3.4.4) gives rise to the following commutative diagram of semi-algebraic maps (see Remarks 11):

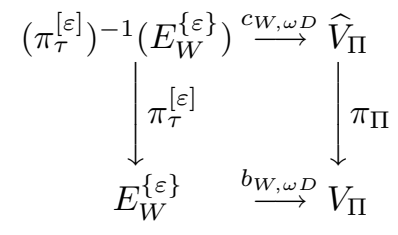

Remark 10. Generally, we have the following result on the real form of linearization map ([S4]). For any twisted real structure $T_{W, \mathbb{R}}^{[u]}$ and for any connected component $E$ of $T_{W, \mathbb{R}}^{[u]} \backslash B_{W, \text { odd }, \mathbb{R}}^{[u]}$, there exists an involution $\chi \in \mathfrak{S}(\Pi)$ such that the linearization map $b_{W, \omega D}$ induces a (real multivalued) map from $E$ to the twisted real space $V_{\Pi}^{\chi}$, where the scaling constant $\omega$ is chosen in the twisted real form: $\mathbb{C}^{\times, b[u]}=\mathbb{R}_{>0} \sqrt{b[u]} \sqcup\left(-\mathbb{R}_{>0} \sqrt{b[u]}\right)$ with respect to the sign $b[u] \in\{ \pm 1\}$ introduced in Assertion 2.1 in $\S 2.3$. 
Actually, in the present paper, we take $[u]=[\varepsilon], E=E_{W}^{\{\varepsilon\}}, b[\varepsilon]=\varepsilon^{h}$, and $\chi=\mathrm{id} \in \mathfrak{S}(\Pi)$.

Remark 11. So far in the present paper, the linearization maps are defined on the covering spaces $T_{W \text {,odd, } \mathbb{C}}$ and $S_{W \text {,odd, } \mathbb{C}}$. Therefore, one should have, first, introduced the map $b_{W, \omega D}$ on a certain covering space $\tilde{E}_{W}^{\{\varepsilon\}}$ embedded in $T_{W, \text { odd, }}$, namely on the connected component of the inverse image of $E_{W}^{\{\varepsilon\}}$ in $T_{W, \text { odd }, \mathbb{C}}$ which contains the base point locus $O_{\text {odd }}^{\varepsilon}$, and similarly for $c_{W, \omega D}$, and then formulate Theorem $\mathrm{C}$ in terms of the map defined on the covering spaces. Actually, as a consequence of Theorem C, $E_{W}^{\{\varepsilon\}}$ become homeomorphic to a simplicial cone in a real vector space so that it is simply connected. Also, $\left(\pi_{\tau}^{[\varepsilon]}\right)^{-1}\left(E_{W}^{\{\varepsilon\}}\right)$, as a tube domain over $E_{W}^{\{\varepsilon\}}$, is simply connected. Thus the covering spaces reduce to trivial covering spaces, and $b_{W, \omega D}$ and $c_{W, \omega D}$ are well defined as univalent maps on $E_{W}^{\{\varepsilon\}}$ and $\left(\pi_{\tau}^{[\varepsilon]}\right)^{-1}\left(E_{W}^{\{\varepsilon\}}\right)$. Therefore, in the formulation of Theorem $\mathrm{C}$ in the present paper, we assume the knowledge of the simply connectedness beforehand.

We state Theorem C, announced in the introduction.

Theorem C. Depending on the choices of the sign $\varepsilon \in\{ \pm 1\}$, the scaling factor $\omega \in\left\{ \pm \sqrt{\varepsilon^{h}}\right\}$ and the partition $\left\{\Pi_{1}, \Pi_{2}\right\}$, take a sign factor

$$
\sigma:=-\omega \sigma\left(D,\left\{\Pi_{1}, \Pi_{2}\right\}\right)(\sqrt{\varepsilon})^{h} \in\{ \pm 1\}
$$

(recall (2.5.4) for $\left.\sigma\left(D,\left\{\Pi_{1}, \Pi_{2}\right\}\right)\right)$. Then the following (1)-(7) hold.

(1) The linearization map $b_{W, \omega D}^{[\varepsilon]}$ induces a semi-algebraic homeomorphism:

$$
b_{W, \sigma \cdot \omega D}^{[\varepsilon]}: E_{W}^{\{\varepsilon\}} \stackrel{\sim}{\longrightarrow} E_{\Gamma(W)},
$$

which extends to their closures homeomorphically.

(2) The linearization map $c_{W, \omega D}^{[\varepsilon]}$ induces a semi-algebraic homeomorphism:

$$
c_{W, \sigma \cdot \omega D}^{[\varepsilon]}:\left(\pi_{\tau}^{[\varepsilon]}\right)^{-1}\left(E_{W}^{\{\varepsilon\}}\right) \stackrel{\sim}{\longrightarrow}\left(\pi_{\Pi}\right)^{-1}\left(E_{\Gamma(W)}\right)
$$

which extends to their closures homeomorphically.

(3) The linearization map (3.5.4) is $\mathbb{G}_{a}$-equivariant so that we obtain the commutative Cartesian diagram:

$$
\begin{aligned}
& \left(\pi_{\tau}^{[\varepsilon]}\right)^{-1}\left(E_{W}^{\{\varepsilon\}} \underset{c_{W, \sigma \cdot \omega D}}{\sim}\left(\pi_{\Pi}\right)^{-1}\left(E_{\Gamma(W)}\right)\right.
\end{aligned}
$$

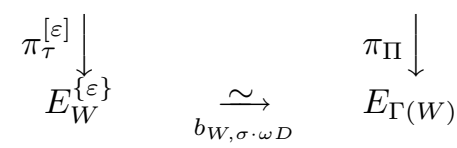


(4) The linearization map $b_{W, \sigma \cdot \omega D}^{[\varepsilon]}$ maps the ordinary bifurcation set $B_{W, 2, \mathbb{R}}^{[\varepsilon]}$ to the union of the 2-labeled reflection hyperplanes in $V_{\Pi}$ :

$$
b_{W, \sigma \cdot \omega D}^{[\varepsilon]}: E_{W}^{\{\varepsilon\}} \cap B_{W, 2, \mathbb{R}} \stackrel{\sim}{\longrightarrow} E_{\Gamma(W)} \cap\left(\cup_{\overline{\alpha \beta} \notin E d g e(\Gamma(W))} H_{\alpha \beta}\right),
$$

(5) The linearization map $c_{W, \sigma \cdot \omega D}^{[\varepsilon]}$ maps the real discriminant locus $D_{W, \mathbb{R}}^{[\varepsilon]}$ to the union of the hyperplanes in $\widehat{V_{\Pi}}$ :

$$
c_{W, \sigma \cdot \omega D}:\left(\pi_{\tau}^{[\varepsilon]}\right)^{-1}\left(\overline{E_{W}^{\{\varepsilon\}}}\right) \cap D_{W, \mathbb{R}}^{[\varepsilon]} \stackrel{\sim}{\longrightarrow}\left(\pi_{\Pi}\right)^{-1}\left(E_{\Gamma(W)}\right) \cap\left(\cup_{\alpha \in \Pi} H_{\alpha}\right),
$$

(6) The linearization map $c_{W, \omega D}^{[\varepsilon]}$ maps the central component $\mathcal{C}^{\{\varepsilon\}}$ in $S_{W, \mathbb{R}}^{[\varepsilon]}$ to the coordinate hyperquadrant in $\widehat{V}_{\Pi}$ :

$$
\widehat{\mathcal{C}}_{\Gamma(W)}:=\left\{\sum_{\alpha \in \Pi} \lambda_{\alpha} v_{\alpha} \in \widehat{V_{\Pi}} \mid(-1)^{i} \lambda_{\alpha}>0 \text { for } \alpha \in \Pi_{i}, i=1,2\right\}
$$

homeomorphically:

$$
c_{W, \sigma \cdot \omega D}^{[\varepsilon]}: \quad \mathcal{C}^{\{\varepsilon\}} \stackrel{\sim}{\longrightarrow} \widehat{\mathcal{C}}_{\Gamma(W)} .
$$

The map extends to their closures homeomorphically. Here, one note that the hyper-quadrant satisfies $\pi_{\Pi}\left(\widehat{\mathcal{C}}_{\Gamma(W)}\right) \subset E_{\Gamma(W)}$, but the equality may not holds.

(7) The linearization map $c_{W, \omega D}^{[\varepsilon]}$ maps the opposite components $\mathcal{C}_{ \pm}^{[\varepsilon]}$ in $S_{W, \mathbb{R}}^{[\varepsilon]}$ (cf. (1.7.1) and (2.5.5)) to the hyper-quadrants in $\widehat{V}_{\Pi}$ :

$$
\begin{aligned}
\widehat{\mathcal{C}}_{\Gamma(W), \pm}:=\left\{\sum_{\alpha \in \Pi} \lambda_{\alpha} v_{\alpha} \in \widehat{V_{\Pi}}\right. & \mid(-1)^{i-1} \lambda_{\alpha}>0 \text { for } i \text { with } \\
& \left.(-1)^{i-1}= \pm 1 \text { and } \alpha \in \Pi_{i}\right\}
\end{aligned}
$$

homeomorphically:

$$
c_{W, \sigma \cdot \omega D}^{[\varepsilon]}: \quad \mathcal{C}_{ \pm}^{[\varepsilon]} \cap\left(\pi_{\tau}^{[\varepsilon]}\right)^{-1} E_{W}^{\{\varepsilon\}} \stackrel{\sim}{\longrightarrow} \widehat{\mathcal{C}}_{\Gamma(W), \pm} \cap\left(\pi_{\Pi}\right)^{-1} E_{\Gamma(W)} .
$$

The map extends to their closures homeomorphically.

(8) For a subset $\Sigma$ of $\Pi$, let $F_{\Sigma}$ be the facet of $\mathcal{C}^{\{\varepsilon\}}$ corresponding to the facet $\cap_{\alpha \in \Sigma}\left\{\lambda_{\alpha}=0\right\} \cap \widehat{\mathcal{C}}_{\Gamma(W)}$ by (3.5.9). Then, for $\beta \in \Sigma^{c}:=\Pi \backslash \Sigma$, $\phi_{\beta}$ is regular on a neighborhood of $F_{\Sigma}$ in $S_{W, \mathbb{R}}^{\{\varepsilon\}}$. One obtains a semi-algebraic isomorphism:

$$
\left(\sigma \omega \cdot \phi_{\beta}\right)_{\beta \in \Sigma^{c}}: F_{\Sigma} \stackrel{\sim}{\longrightarrow}\left(\prod_{\beta \in \Pi_{1} \cap \Sigma^{c}} \mathbb{R}_{>0}\right) \times\left(\prod_{\beta \in \Pi_{2} \cap \Sigma^{c}} \mathbb{R}_{<0}\right)
$$

Remark 12. In Theorem C, the scaling factor $\omega$ and the sign factor $\sigma$ appear always as the product $\sigma \cdot \omega$. In this paper we distinguished them because of their different origins. 


\section{§3.6. Proof of Theorem A}

Recall the $\mathbb{R}$-equivariant isomorphism (3.5.5). Using (3.5.8)-(3.5.11), for a positive real number $\lambda \in \mathbb{R}_{>0}$, the map $c_{W, \sigma \cdot \omega D}^{[\varepsilon]}$ induces a semi-algebraic diffeomorphism from $\bar{J}_{W}^{\{\varepsilon\}}(\lambda):=\overline{\mathcal{C}}^{\{\varepsilon\}} \cap \tau^{[\varepsilon]}(-\lambda) \overline{\mathcal{C}}_{+}^{[\varepsilon]} \cap \tau^{[\varepsilon]}(\lambda) \overline{\mathcal{C}}_{-}^{[\varepsilon]}$ to

$$
\begin{aligned}
& \widehat{\mathcal{C}}_{\Gamma(W)} \cap\left(\widehat{\mathcal{C}}_{\Gamma(W),+}-\lambda v_{\Pi}\right) \cap\left(\widehat{\mathcal{C}}_{\Gamma(W),-}+\lambda v_{\Pi}\right) \\
= & \left\{\left(\lambda_{\alpha}\right)_{\alpha \in \Pi} \in \widehat{V_{\Pi}} \mid 0 \leq(-1)^{i} \lambda_{\alpha} \leq \lambda \text { for } \alpha \in \Pi_{i}, i=1,2\right\}
\end{aligned}
$$

where the right hand side is a parallelotope of dimension $l$ in $\widehat{V_{\Pi}}$. It is the intersection of two simplicial cones $\widehat{\mathcal{C}}_{\Gamma(W)}^{\varepsilon}$ and $\widehat{\mathcal{C}}_{\Gamma(W)}^{\varepsilon}(\lambda)$, where

$$
\begin{aligned}
\widehat{\mathcal{C}}_{\Gamma(W)}^{\varepsilon}(\lambda): & =\left(\widehat{\mathcal{C}}_{\Gamma(W),+}-\lambda v_{\Pi}\right) \cap\left(\widehat{\mathcal{C}}_{\Gamma(W),-}+\lambda v_{\Pi}\right) \\
& =\left\{\left(\lambda_{\alpha}\right)_{\alpha \in \Pi} \in \widehat{V_{\Pi}} \mid(-1)^{i} \lambda_{\alpha} \leq \lambda \text { for } \alpha \in \Pi_{i}, i=1,2\right\} .
\end{aligned}
$$

Let us show a slightly stronger transversality between these two cones in order to apply it to the proof of Theorem B in the next subsection.

Theorem A addendum. The faces of $\bar{J}_{W}^{\{\varepsilon\}}(\lambda)$ are crossing normally at any point of $\bar{J}_{W}^{\{\varepsilon\}}(\lambda) \backslash\left(\pi_{\tau}^{[\varepsilon]}\right)^{-1}\left(B_{W, \geq 3, \mathbb{R}}^{[\varepsilon]}\right)$ (recall $\S 1.3$ Definition 1 .).

Proof. Recall the formula (3.4.5) for the Jacobian $\frac{\partial\left(\phi_{\alpha_{1}}, \ldots, \phi_{\alpha_{l}}\right)}{\partial\left(P_{1}, \ldots, P_{l}\right)}$ of the map $c_{W}$. The right hand expression means that it does not vanish on the complement of $\left(\pi_{\tau}\right)^{-1}\left(B_{W, \geq 3, \mathbb{R}}^{[\varepsilon]}\right)$. This means that the hyperplanes $\phi_{\alpha}=$ const for $\alpha \in$ $\Pi$, which define faces of the polyhedra $J_{W}^{\{\varepsilon\}}(\lambda)$, are normally crossing on the complement of $\left(\pi_{\tau}\right)^{-1}\left(B_{W, \geq 3, \mathbb{R}}^{[\varepsilon]}\right)$.

This addendum proves the transversality stated in Theorem A and hence completes the proof of Theorem A.

Remark 13. Let $a o^{\{\varepsilon\}}(\lambda)$ be the vertex of $\bar{J}_{W}^{\{\varepsilon\}}(\lambda)$ which is antipodal to

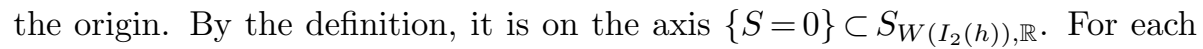
$\varepsilon \in\{ \pm 1\}$, one has $A O^{\varepsilon}=\left\{a o^{\{\varepsilon\}}(\lambda) \mid \lambda \in \mathbb{R}_{>0}\right\}$. This is the reason why $A O^{\varepsilon}$ introduced in (2.5.6), is called the vertex orbit axis.

\section{§3.7. Proof of Theorem B}

We shall show that

$$
\bar{K}_{W}^{\varepsilon}(\lambda):=\left(\pi_{W}\right)^{-1}\left(\bar{J}_{W}^{\{\varepsilon\}}(\lambda)\right)
$$

is a semi-algebraic polyhedron dual to the Weyl chamber decomposition of $V_{\mathbb{R}}^{\varepsilon}$. Since the proofs for $\varepsilon=1$ and for $\varepsilon=-1$ are completely parallel, we prove only the case $\varepsilon=1$ and omit the upperscripts $\varepsilon,\{\varepsilon\}$ and $[\varepsilon]$. The proof is divided into two parts: 1. local analytic part and 2. global combinatorial part. 
1. We study the local analytic property of $\bar{K}_{W}(\lambda)$. In this paragraph, we mean by $(X, x) \simeq(Y, y)$ that there exists an analytic isomorphism from a neighborhood of $x$ in $X$ to a neighborhood of $y$ in $Y$ bringing $x$ to $y$.

Assertion 3.3. Let $\tilde{x} \in \bar{K}_{W}(\lambda)$ and $m:=\operatorname{dim}_{\mathbb{R}} V_{\mathbb{R}}^{W(\tilde{x})}$, where $W(\tilde{x})$ is the stabilizer subgroup of $W$ at $\tilde{x}$ and $V_{\mathbb{R}}^{W(\tilde{x})}$ is the fixed point subspace by the $W(\tilde{x})$-action. Then, there exist an integer $k$ with $0 \leq k \leq m$ and a local real analytic isomorphism from a neighborhood of $\tilde{x}$ in $V_{\mathbb{R}}$ to a neighborhood of the origin of $\left(V_{\mathbb{R}} / V_{\mathbb{R}}^{W(\tilde{x})}\right) \times \mathbb{R}^{m}$ which makes the following diagram commutative:

$$
\begin{gathered}
\left(V_{\mathbb{R}}, \tilde{x}\right) \simeq\left(\left(V_{\mathbb{R}} / V_{\mathbb{R}}^{W(\tilde{x})}\right) \times \mathbb{R}^{m}, 0\right) \\
\cup \\
\left(\bar{K}_{W}(\lambda), \tilde{x}\right) \simeq\left(\left(V_{\mathbb{R}} / V_{\mathbb{R}}^{W(\tilde{x})}\right) \times \mathbb{R}_{\geq 0}^{k} \times \mathbb{R}^{m-k}, 0\right),
\end{gathered}
$$

Furthermore, the isomorphism induces the following isomorphisms:

i) the isomorphism of the subspaces

$$
\left(V_{\mathbb{R}}^{W(\tilde{x})}, x\right) \simeq\left(\{0\} \times \mathbb{R}^{m}, 0\right)
$$

ii) for any facet $G$ of $\bar{J}_{W}(\lambda)$ which is adjacent to ao $(\lambda)$

$$
\left(\pi_{W}^{-1}(\bar{G}), \tilde{x}\right) \simeq\left(\left(V_{\mathbb{R}} / V_{\mathbb{R}}^{W(\tilde{x})}\right) \times \bar{K}, 0\right)
$$

where $\bar{K}$ is the closure of a facet $K$ of $\left(\mathbb{R}_{\geq 0}^{k} \times \mathbb{R}^{m-k}, 0\right)$ ( which may be empty).

Proof. Let $Q_{1}, \ldots, Q_{l}$ be a system of generators of the ring of invariants $S\left(V_{\mathbb{R}}^{*}\right)^{W(\tilde{x})}$, and let us consider a $W(\tilde{x})$-invariant map $\bar{Q}=\left(Q_{1}, \ldots, Q_{l}\right)$ : $\left(V_{\mathbb{R}}, \tilde{x}\right) \rightarrow\left(\mathbb{R}^{l}, \bar{x}\right)$ for $\bar{x}:=\bar{Q}(\tilde{x})$. Then, there is a local analytic isomorphism $\varpi:\left(\mathbb{R}^{l}, \bar{x}\right) \simeq\left(S_{W, \mathbb{R}}, x\right)$ for $x:=\pi_{W}(\tilde{x})$ such that $\pi_{W}=\varpi \circ \bar{Q}$. We may choose the first $Q_{1}, \ldots, Q_{l-m}$ to be the generators of the ring of invariant polynomials on $V_{\mathbb{R}} / V_{\mathbb{R}}^{W(\tilde{x})}$ by the $W(\tilde{x})$-action, and the last $Q_{l-m+1}, \ldots, Q_{l}$ to be $W(\tilde{x})$-invariant linear functions on $V_{\mathbb{R}}$ whose restrictions on $V_{\mathbb{R}}^{W(\tilde{x})}$ give its linear coordinate system such that $\bar{x}=0$. By this choice of the $Q_{i}$ 's, the local analytic isomorphism $\varpi^{-1}$ induces a local splitting of the set $\mathcal{C}$ at $x$ :

$$
(\overline{\mathcal{C}}, x) \simeq\left(\left(\left(V_{\mathbb{R}} / V_{\mathbb{R}}^{W(\tilde{x})}\right) / W(\tilde{x})\right) \times \mathbb{R}^{m}, 0\right) .
$$

We shall denote by $F$ the stratum of $\overline{\mathcal{C}}$ containing $x$ in the left hand side, which is locally the image of $\left(V_{\mathbb{R}}^{W(\tilde{x})}, \tilde{x}\right)$ by $\pi_{W}$. Then $(F, x)$ is mapped to the subspace $\left(\mathbb{R}^{m}, 0\right)$ in the right hand side. 
On the other hand, the linearization map $c_{W}$ maps the central component $\mathcal{C}$ to the cone $\widehat{\mathcal{C}}_{\Gamma(W)}(3.5 .8)$, and hence the stratum $F$ to a stratum of $\widehat{\mathcal{C}}_{\Gamma(W)}$, which is an open cone in $\cap_{i=m+1}^{l} H_{\alpha_{i}}$ for some $\left\{\alpha_{m+1}, \ldots, \alpha_{l}\right\} \subset \Pi$. Then, $\phi_{\alpha_{1}}, \ldots, \phi_{\alpha_{m}}$ for the remaining index set $\left\{\alpha_{1}, \ldots, \alpha_{m}\right\}=\Pi \backslash\left\{\alpha_{1}, \ldots, \alpha_{l-m}\right\}$ form a local coordinate system of $F$ at $x$ (Theorem C (8)). Therefore, replacing $Q_{l-m+1}, \ldots, Q_{l}$ with $\phi_{\alpha_{1}}-\phi_{\alpha_{1}}(x), \ldots, \phi_{\alpha_{m}}-\phi_{\alpha_{m}}(x)$, we obtain a local analytic expression similar to (3.7.5), where the subspace $\left(\{0\} \times \mathbb{R}^{m}, 0\right)$ of the right hand side is still the image of $F$ by $\left(\phi_{\alpha_{i}}-\phi_{\alpha_{i}}(x)\right)$.

The parallelotope $\bar{J}_{W}$, locally at $x$, is defined as the subset of the central component $\overline{\mathcal{C}}$ given by inequalities $\pm \phi_{\alpha_{i}} \leq \lambda$ for some $i \in\{1, \ldots, m\}$ and suitable signs (depending on $i$, recall (3.6.1)). Then after a suitable renumbering of $\{1, \ldots, m\}$, we obtain further a local isomorphism:

$$
\left(\bar{J}_{W}(\lambda), x\right) \simeq\left(\left(\left(V_{\mathbb{R}} / V_{\mathbb{R}}^{W(\tilde{x})}\right) / W(\tilde{x})\right) \times \mathbb{R}_{\geq 0}^{k} \times \mathbb{R}^{m-k}, 0\right) .
$$

The facet decomposition of $\bar{J}_{W}(\lambda)$ as a parallelotope at $x$ coincides with the natural facet decomposition of $\mathbb{R}_{\geq 0}^{k} \times \mathbb{R}^{m-k}$ in the right hand side.

Taking the inverse images of the both sides of (3.7.6) in their covering spaces, i.e., a neighborhood of $\tilde{x}$ in $V_{\mathbb{R}}$ and a neighborhood of the origin in $\left(V_{\mathbb{R}} / V_{\mathbb{R}}^{W(\tilde{x})}\right) \times \mathbb{R}^{m}$, respectively, we obtain the local analytic isomorphism (3.7.2). Then (3.7.3) follows from the construction.

Let us consider a facet $G$ of $\bar{J}_{W}$ which is adjacent to $a o(\lambda)$. Since $a o(\lambda) \in$ $\bar{G}, G$ is contained in the interior of $\overline{\mathcal{C}}$. This implies the image of $G$ in the right hand side of $(3.7 .5)$ is contained in $\left(\left(\left(V_{\mathbb{R}} / V_{\mathbb{R}}^{W(\tilde{x})}\right) / W(\tilde{x})\right)^{\circ} \times \mathbb{R}^{m}, 0\right)$ where $\left(\left(V_{\mathbb{R}} / V_{\mathbb{R}}^{W(\tilde{x})}\right) / W(\tilde{x})\right)^{\circ}$ is the unique open facet. Then, by the isomorphism (3.7.6), the closure $\bar{G}$ of the stratum $G$ is mapped to $\left(\left(\left(V_{\mathbb{R}} / V_{\mathbb{R}}^{W(\tilde{x})}\right) / W(\tilde{x})\right) \times\right.$ $\bar{K}, 0)$ for the closure of a suitable facet $K$ of $\left(\mathbb{R}_{\geq 0}^{k} \times \mathbb{R}^{m-k}, 0\right)$ (including empty case). By taking their inverse images, the isomorphism in the first line of (3.7.2) induces (3.7.4).

Corollary. Let $G$ be a facet of $\bar{J}_{W}(\lambda)$ which is adjacent to ao $(\lambda)$. Then $\left(\pi_{W}\right)^{-1}(\bar{G})$ is a submanifold with corners in $V_{\mathbb{R}}$, which is transversal to the system of hyperplanes $\left\{H_{\alpha, \mathbb{R}}\right\}_{\alpha \in R(W)}$.

Proof. Since $\left.\pi_{W}\right|_{\left(V_{\mathbb{R}} \backslash \cup_{\alpha \in R(W)} H_{\alpha, \mathbb{R}}\right)}$ is locally biregular and $\bar{G} \backslash D_{W, \mathbb{R}}$ is a manifold with corner due to Theorem A addendum in $\S 3.6$, we only have to show the property of $\left(\pi_{W}\right)^{-1}(\bar{G})$ at a point $\tilde{x} \in\left(\cup_{\alpha \in R(W)} H_{\alpha, \mathbb{R}}\right) \cap\left(\pi_{W}\right)^{-1}(\bar{G})$. Apply Assertion 3.3 at the point $\tilde{x}$. 
The fact ii) in Assertion 3.3 implies that $\left(\pi_{W}\right)^{-1}(\bar{G})$ is a locally closed manifold with corners. Furthermore, the fact that $\left(\left(\pi_{W}\right)^{-1}(\bar{G}), \tilde{x}\right)$ contains the factor $\left(V_{\mathbb{R}} / V_{\mathbb{R}}^{W(\tilde{x})}, 0\right)$ implies that it is transversal to the submanifold $\left(\mathbb{R}^{m}, 0\right)$. Since $V_{\mathbb{R}}^{W(\tilde{x})}$ is the intersection of the reflection hyperplanes pathing through $\tilde{x}, \mathrm{i})$ in Assertion 3.3 implies that $\left(\left(\pi_{W}\right)^{-1}(\bar{G}), \tilde{x}\right)$ is transversal to every facet $V_{\gamma}$ of $V_{\mathbb{R}}($ recall $(1.2 .2))$.

2. We describe the facet decomposition of $\bar{K}_{W}(\lambda):=\left(\pi_{W}\right)^{-1}\left(\bar{J}_{W}(\lambda)\right)$. We first prepare terminology on the facet decomposition of $\bar{J}_{W}$.

Let $\mathcal{F}(o)=\left\{F_{\bar{\gamma}}\right\}_{\bar{\gamma} \in \Gamma(o)}$ and $\mathcal{F}(a o)=\left\{G_{\bar{\delta}}\right\}_{\bar{\delta} \in \Gamma(o)}$ be the sets of facets of $\bar{J}_{W}$ which are adjacent to $o$ and to $a o$, respectively. Here we use the same index set $\Gamma(o)$ for the both sets by the reason i) below, and put an overline on the index by the reason iv) below.

i) There is a one-to-one correspondence $\mathcal{F}(o) \leftrightarrow \mathcal{F}(a o)$ in such a manner that $F_{\bar{\gamma}} \leftrightarrow G_{\bar{\delta}}$ if and only if $\bar{F}_{\bar{\gamma}} \cap \bar{G}_{\bar{\delta}}$ consists of a single point.

ii) The set $\Gamma(o)$ is partially ordered such that for $\bar{\gamma}, \bar{\delta} \in \Gamma(o)$ one has

$$
\bar{\gamma} \leq \bar{\delta} \quad \Leftrightarrow \quad F_{\bar{\gamma}} \subset \bar{F}_{\bar{\delta}} \Leftrightarrow \bar{G}_{\bar{\gamma}} \supset G_{\bar{\delta}} .
$$

iii) $\bar{G}_{\bar{\gamma}} \cap \bar{F}_{\bar{\delta}} \neq \emptyset$ if and only if $\bar{\gamma} \leq \bar{\delta}$ for $\bar{\gamma}, \bar{\delta} \in \Gamma(o)$. The intersection is a closed facet of $\bar{J}_{W}\left(\simeq[0,1]^{k}\right)$ of dimension $k=\operatorname{dim}\left(F_{\bar{\delta}}\right)-\operatorname{dim}\left(F_{\bar{\gamma}}\right)$.

iv) Recall the index set $\Gamma$ (1.2.2), on which $W$ acts in the obvious manner. Then, there is a bijection $\Gamma / W \simeq \Gamma(o)$ (where the image of $\delta \in \Gamma$ is denoted by $\bar{\delta} \in \Gamma(o))$ such that $\pi_{W}\left(\bar{V}_{\delta}\right) \cap \bar{J}_{W}(\lambda)=\bar{F}_{\bar{\delta}}$ for $\delta \in \Gamma$.

Definition. A semi-algebraic set $K$ in $V_{\mathbb{R}}$ is called a facet of $\bar{K}_{W}(\lambda)$ if there is $G_{\bar{\gamma}} \in \mathcal{F}(a o)$ such that $K$ is the interior of a connected component of $\left(\pi_{W}\right)^{-1}\left(\bar{G}_{\bar{\gamma}}\right)$.

Let us show that the set of all facets of $\bar{K}_{W}(\lambda)$ is indexed by $\Gamma$. For any $\gamma \in$ $\Gamma$, by the definition, $\pi_{W}\left(V_{\gamma}\right)$ and $\bar{G}_{\bar{\gamma}}$ intersects at a single point transversally. Therefore, there exists a unique connected component of $\left(\pi_{W}\right)^{-1}\left(\bar{G}_{\bar{\gamma}}\right)$ that intersects $V_{\gamma}$. Its interior is, by the definition, a facet of $\bar{K}_{W}(\lambda)$, which we shall denote by $K_{\gamma}$. Conversely, let $K$ be the interior of a connected component of $\left(\pi_{W}\right)^{-1}\left(\bar{G}_{\bar{\gamma}}\right)$ for some $\bar{\gamma} \in \Gamma(o)$. Since $\bar{F}_{\bar{\gamma}}$ intersects $\bar{G}_{\bar{\gamma}}$ transversally at a point, there exists a connected component of $\left(\pi_{W}\right)^{-1}\left(F_{\bar{\gamma}}\right)$ whose closure intersects $K$ at a single point. Let $V_{\gamma}$ for $\gamma \in \Gamma$ be the cone in (1.2.2) which support the component such that $\gamma$ projects to $\bar{\gamma}$. Thus, we find a unique $\gamma \in \Gamma$ such that $V_{\gamma}$ intersect $K$ at a point. 
By definition, all facets of $\bar{K}_{W}(\lambda)$ are disjoint and cover $\bar{K}_{W}(\lambda)$. This shows the decomposition

$$
\bar{K}_{W}(\lambda)=\sqcup_{\gamma \in \Gamma} K_{\gamma}
$$

The fact that (3.7.8) gives a semi-algebraic stratification of $\bar{K}_{W}(\lambda)$ (i.e., they satisfy the boundary condition) can be reduced to that of $\mathcal{F}(a o)$. The fact that $\bar{K}_{W}(\lambda)$ becomes a semi-algebraic polyhedron with respect to the decomposition (3.7.8) whose faces are normally crossing follows from (3.7.2).

Finally, we have to show the three duality properties i), ii) and iii) for $\bar{K}_{W}(\lambda)$ in Definition 2 in $\S 1.3$. The proofs are reduced to the duality between $\mathcal{F}(o)$ and $\mathcal{F}(a o)$ and to the local analysis discussed in the first half of this proof. In particular, for the last iii), we have to show that the intersection of the closure of a chamber $C$ with the $\bar{K}_{W}(\lambda)$

$$
\bar{C} \cap \bar{K}_{W}(\lambda)
$$

is analytically isomorphic to the cube $[0,1]^{l}$ in the following strong sense: there is a neighborhood in $V_{\mathbb{R}}$ of the set (3.7.9) and a real analytic isomorphism of the neighborhood to an open subset of $\mathbb{R}^{l}$ such that the set (3.7.9) is mapped homeomorphically onto the cube $[0,1]^{l}$. The fact that the set (3.7.9) is homeomorphic to a cube follows from the fact that the restriction of $\pi_{W}$ on the set (3.7.9) is a homeomorphism onto the polyhedron $\bar{J}_{W}(\lambda)$. On the other hand, we have shown in Assertion 3.3 that faces are normally crossing at any point of the boundary of the set (3.7.9). These two show the required result.

These complete the proof of Theorem B.

Note. $\quad$ The set (3.7.9) is given by two systems of $l$-inequalities on $V_{\mathbb{R}}$.

$$
\left(\cap_{\alpha \in \Pi}\left\{f_{\alpha} \geq 0\right\}\right) \cap\left(\cap_{i=1,2} \cap_{\alpha \in \Pi_{i}}\left\{(-1)^{i} \phi_{\alpha} \leq \lambda\right\}\right) .
$$

\section{§3.8. Examples of type $A_{3}$}

The shaded area in the upper and lower right figures (Fig. 2) are the central region $E_{A_{3}}^{\{\varepsilon\}}$ and the $\Gamma\left(A_{3}\right)$-cone $E_{\Gamma\left(A_{3}\right)}$, respectively. The tube domain $\left(\pi_{\tau}^{[\varepsilon]}\right)^{-1}\left(E_{A_{3}}^{\{\varepsilon\}}\right)$ in $S_{A_{3}, \mathbb{R}}^{[\varepsilon]}$ is illustrated in the upper left figure as the domain sandwiched by the covers of an open booklet. The tube domain $\pi_{\Pi}^{-1}\left(E_{\Gamma\left(A_{3}\right)}\right)$ in $\widehat{V_{\Pi}}$ is illustrated in the lower left figure as the domain sandwiched by the straight covers of an open booklet. 

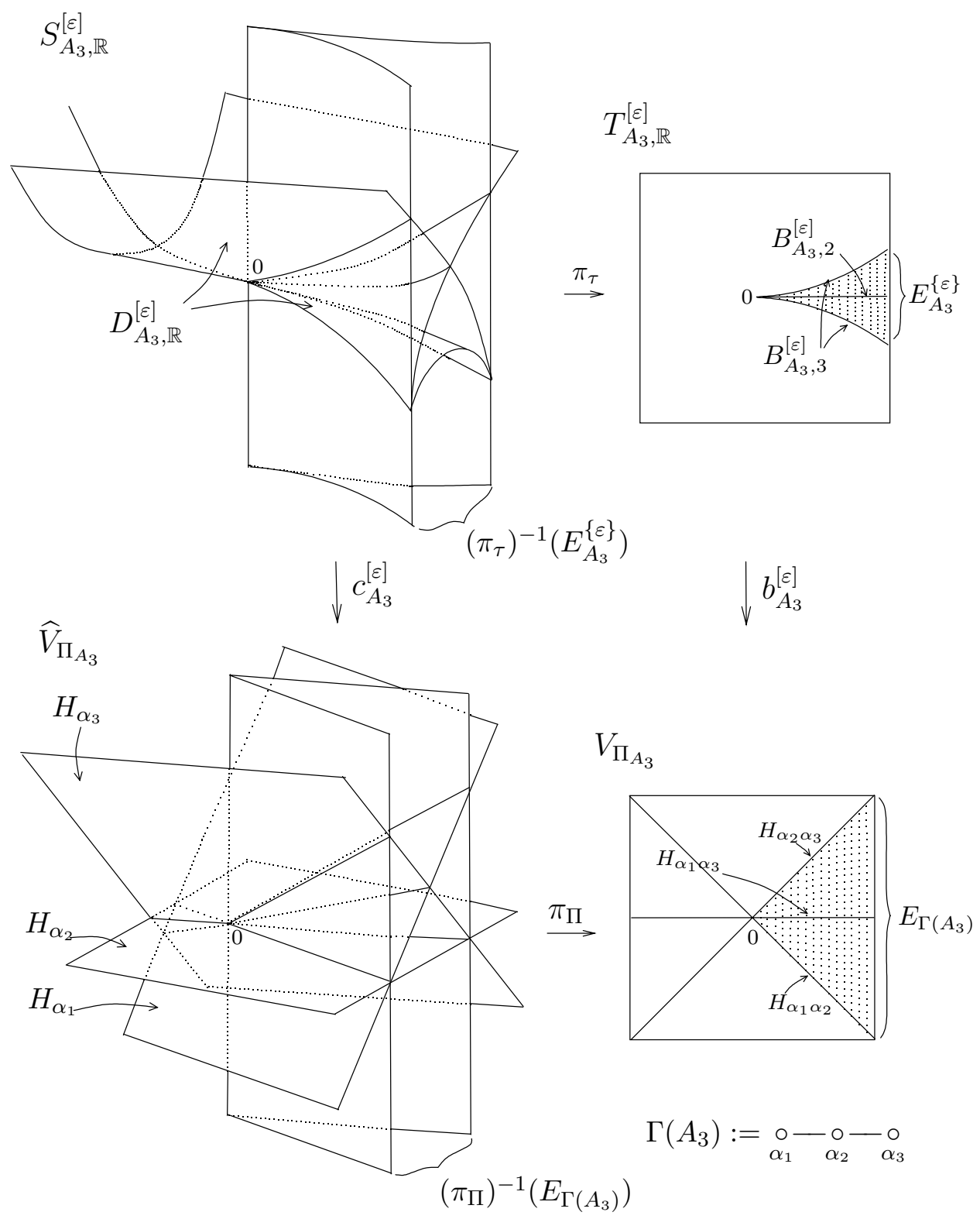

Figure 2. The linearization maps of type $A_{3}$ 


\section{$\S 4$. Fundamental Group of $S_{W, \mathbb{C}} \backslash D_{W, \mathbb{C}}$}

An Artin group presentation of the fundamental group of the regular orbit space $S_{W, \mathbb{C}} \backslash D_{W, \mathbb{C}}$ is given by E. Brieskorn [Br1], where a group $G$ with the presentation: generator system $a_{\alpha}(\alpha \in \Pi)$ and the braid relations: $\underbrace{a_{\alpha} a_{\beta} \ldots}=$ $\underbrace{a_{\beta} a_{\alpha} \ldots}_{m_{\alpha \beta} \text {-letters }}$ for $\alpha, \beta \in \Pi$ as for the fundamental relations, is called an Artin group [BS]. The generators of $\pi_{1}\left(S_{W, \mathbb{C}} \backslash D_{W, \mathbb{C}}\right)$ are described in [Br1] in terms of adjacent chambers in $V_{\mathbb{R}}$ (see also a work by P. Deligne [D1], where the Artin group is given in terms of the galleries of chambers). Then, several authors including Brieskorn, Deligne and the author asked to describe the generator system of the Artin group in terms of a geometry on $S_{W, \mathbb{C}}$.

As a consequence of Theorems A, B and C, we give two different answers to this question. The first one is to use the 1-skeleton of $J_{W}(\lambda)^{\{\varepsilon\}}$ and is described in 4.1. Identification with Brieskorn's generator system is given in 4.2. The second one is to use $\tau$-orbits as the pencil of Zariski-van Kampen method. It is described in $\S 4.3$ and is identified in $\S 4.4$ with the one given in $\S 4.1$. The generator systems, we have described, depend on the choice of $\varepsilon \in\{ \pm 1\}$ since the base points belong to the different central component $\mathcal{C}^{\{\varepsilon\}}$. The relation between the two generator systems is given in 4.5 .

In this section, we sometimes identify a path and its homotopy class.

\section{§4.1. 1-skeleton of the polyhedron $J_{W}^{\{\varepsilon\}}(\lambda)$}

With a use of the 1-skeleton of the polyhedron $J_{W}^{\{\varepsilon\}}(\lambda)$ (Theorem A), we construct a generator system of $\pi_{1}\left(S_{W, \mathbb{C}} \backslash D_{W, \mathbb{C}}\right)$. Using Theorem B, they are identified in $\S 4.2$ with the one studied by Brieskorn $[\mathrm{Br}]$.

Let $a o^{\{\varepsilon\}}(\lambda)$ be the vertex of $J_{W}^{\{\varepsilon\}}(\lambda)$ which is antipodal to the origin (belonging to $A O^{\varepsilon}$ ). Due to Assertion $\S 1.15$, for each $\alpha \in \Pi$, there exists a unique edge $\left[a o^{\{\varepsilon\}}(\lambda), p_{\alpha}\right]$ of $J_{W}^{\{\varepsilon\}}(\lambda)$ which starts from $a o^{\{\varepsilon\}}(\lambda)$ and terminates at a point $p_{\alpha}$ on the $\alpha$ th face of $\overline{\mathcal{C}}^{\{\varepsilon\}}$ (cf. Fig. 4 ).

Since the edge $\left[a o^{\{\varepsilon\}}(\lambda), p_{\alpha}\right]$ intersects the discriminant $D_{W, \mathbb{R}}^{[\varepsilon]}$ transversally at $p_{\alpha}$ (Theorem A), a complexification of $\left[a o^{\{\varepsilon\}}(\lambda), p_{\alpha}\right]$ (a complex open curve in $S_{W, \mathbb{C}}$ which contains $\left.\left[a o^{\{\varepsilon\}}(\lambda), p_{\alpha}\right]\right)$ intersects the discriminant locus $D_{W, \mathbb{C}}$ transversally at $p_{\alpha}$. In the complexification, let us consider a closed path $\gamma_{\alpha}$ based at $a o^{\{\varepsilon\}}(\lambda)$ and turning once around the discriminant locus at $p_{\alpha}$ counterclockwise as in Fig. 3. 


$$
p_{\alpha}=\underbrace{\left(\lambda, \lambda, \cdots, \stackrel{\alpha}{0, \cdots, \lambda) \quad a o^{\{\varepsilon\}}}(\lambda)\right.}_{\gamma_{\alpha}}(\lambda, \cdots, \lambda)
$$

Figure 3. The generator $\gamma_{\alpha}$ on an edge of $J_{W}^{\{\varepsilon\}}(\lambda)$ (cf. Fig. 4).

Theorem 4.1. The system of the homotopy classes of $\gamma_{\alpha}(\alpha \in \Pi)$ in $\pi_{1}\left(S_{W, \mathbb{C}} \backslash D_{W, \mathbb{C}}, a o^{\{\varepsilon\}}(\lambda)\right)$ coincides with the generator system $\left\{g_{\alpha}\right\}_{\alpha \in \Pi}$ given by Brieskorn $\left[\right.$ Br1, Zusatz]. Therefore, $\pi_{1}\left(S_{W, \mathbb{C}} \backslash D_{W, \mathbb{C}}, a o^{\{\varepsilon\}}(\lambda)\right)$ is an Artin group with respect to the generator system $\gamma_{\alpha}(\alpha \in \Pi)$.

\section{§4.2. $\quad$ Proof of Theorem 4.1}

Let $\widetilde{a o}^{\varepsilon}(\lambda)$ be the vertex of $K_{W}^{\varepsilon}(\lambda)$ in the chamber $C^{\varepsilon}$ which projects to the vertex $a o^{\varepsilon}(\lambda)$ of $J_{W}^{[\varepsilon]}(\lambda)$. For $\alpha \in \Pi$, let $\alpha \cdot \widetilde{a o}^{\varepsilon}(\lambda)$ be the image of $\widetilde{a o}^{\varepsilon}(\lambda)$ by the reflection $\alpha$, and $\left[\widetilde{a o}^{\varepsilon}(\lambda), \alpha \cdot \widetilde{a o}^{\varepsilon}(\lambda)\right]$ be the 1-edge of the polyhedron $K_{W}^{\varepsilon}(\lambda)$ connecting two vertices $\widetilde{a o}^{\varepsilon}(\lambda)$ and $\alpha \cdot \widetilde{a O} \varepsilon(\lambda)$, which intersects $H_{\alpha, \mathbb{R}}^{\varepsilon}$ transversally at an inverse image $\widetilde{p}_{\alpha}$ of the point $p_{\alpha}$. Then, $\pi_{W}$ projects $\left[\widetilde{a O}^{\varepsilon}(\lambda), \widetilde{p}_{\alpha}\right]$ and $\left[\widetilde{p}_{\alpha}, \alpha \cdot \widetilde{a O}^{\varepsilon}(\lambda)\right]$ onto the edge $\left[p_{\alpha}, a o^{\varepsilon}(\lambda)\right]$.

The inverse image of the path $\gamma_{\alpha}$ (see Fig. 3), which starts at $\widetilde{a o}(\lambda)$, is a path in the complexification of the edge $\left[\widetilde{a O}^{\varepsilon}(\lambda), \alpha \widetilde{a O^{\varepsilon}}(\lambda)\right]$ connecting $\widetilde{a O^{\varepsilon}}(\lambda)$ and $\alpha \cdot \widetilde{a O}^{\varepsilon}(\lambda)$ described as follows: start at $\widetilde{a O}^{\varepsilon}(\lambda)$ and move along $\left[\widetilde{a O}^{\varepsilon}(\lambda), \widetilde{p}_{\alpha}\right]$ close to $\widetilde{p}_{\alpha}$. Then, just before reaching $\widetilde{p}_{\alpha}$ turn along a half circle centered at $\widetilde{p}_{\alpha}$ in the counter-clockwise direction (inside a complexification of $\left[\widetilde{a O}^{\varepsilon}(\lambda), \alpha \cdot \widetilde{a o^{\varepsilon}}(\lambda)\right]$, in which $\left[\widetilde{a O}^{\varepsilon}(\lambda), \alpha \cdot \widetilde{a O}^{\varepsilon}(\lambda)\right]$ crosses the discriminant locus at the point $\left.\widetilde{p}_{\alpha}\right)$ and then to come back to a point $\left[\widetilde{p}_{\alpha}, \alpha \cdot \widetilde{a o}^{\varepsilon}(\lambda)\right]$. Then, again move along $\left[\widetilde{p}_{\alpha}, \alpha \cdot \widetilde{a o}^{\varepsilon}(\lambda)\right]$ till the point $\alpha \cdot \widetilde{a o^{\varepsilon}}(\lambda)$. In fact, this path is homotopic to the path $g_{\alpha}$ described by Brieskorn [Br1,Zusatz].

Note. Let us briefly explain how the braid relations follow immediately from the description of $\gamma_{\alpha}$. For any pair $\alpha, \beta \in \Pi$, consider the 2-dimensional facet, denoted by $\left[a o^{\varepsilon}(\lambda), p_{\alpha}, p_{\beta}\right]$, of ${\overline{J_{W}}}^{\{\varepsilon\}}(\lambda)$ containing the edges $\left[a o^{\varepsilon}(\lambda), p_{\alpha}\right]$ and $\left[a o^{\varepsilon}(\lambda), p_{\beta}\right]$ (Fig. 4.). The $\left[a o^{\varepsilon}(\lambda), p_{\alpha}, p_{\beta}\right]$ is a parallelogram transversal to the 2-codimensional stratum of the discriminant locus of label $m_{\alpha, \beta}$. The inverse image of the parallelogram in $K_{W}^{\varepsilon}(\lambda)$ is a union of $2 m_{\alpha \beta}$-gons, whose boundary are $m_{\alpha \beta^{-}}$-alternating sequence of inverse images of $\left[a o^{\varepsilon}(\lambda), p_{\alpha}\right]$ and $\left[a o^{\varepsilon}(\lambda), p_{\beta}\right]$. One translates a $2 m_{\alpha \beta}$-gon $K_{\alpha \beta}$ to $\widetilde{K}_{\alpha \beta}$ in a complex direction in $V_{\mathbb{C}}$ such that i) $\widetilde{K}_{\alpha \beta}$ does not meat with reflection hyperplanes, ii) the boundary of $\widetilde{K}_{\alpha \beta}$ is homotopic to an alternating sequence of inverse images $\gamma_{\alpha}$ and of $\gamma_{\beta}$. Taking care of the orientations of the edges, one obtains the homotopy relation: $\gamma_{\alpha} \gamma_{\beta} \ldots=\gamma_{\beta} \gamma_{\alpha} \ldots\left(m_{\alpha \beta}\right.$-terms $)$. 


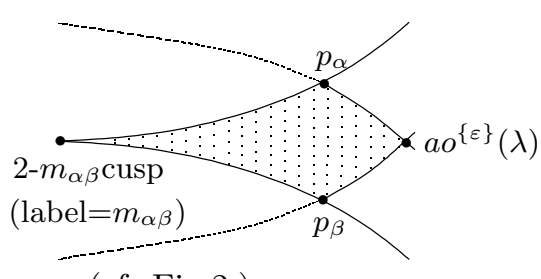

(cf. Fig.2.)

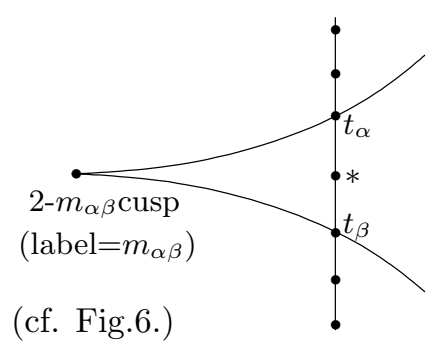

Figure 4 . The 2-Facet $\left[a o^{\varepsilon}(\lambda), p_{\alpha}, p_{\beta}\right] \quad$ Figure 5 . Pencil close to $\overline{\alpha \beta}$-edge

\section{§4.3. Zariski-van Kampen generator system}

We identify the generator system $\left\{\gamma_{\alpha}\right\}_{\alpha \in \Pi}$ in $\S 4.1$ with the well-known Zariski-van Kampen generator system. This is achieved since the $\tau$-direction is transversal to the discriminant divisor, and hence the $\tau$-orbits in $S_{W}$ play a role as the Zariski pencil.

Choose a base point $*^{\varepsilon}$ in the central component $\mathcal{C}^{\{\varepsilon\}}$ in $S_{W, \mathbb{R}}^{[\varepsilon]}$. The real line $\tau^{[\varepsilon]}(\mathbb{R})\left(*^{\varepsilon}\right)$ and the real discriminant locus $D_{W, \mathbb{R}}^{[\varepsilon]}$ intersect by $l$-points (counted with multiplicity) due to Theorem $\mathrm{C}$. We order them as

$$
t_{1} \leq \cdots \leq t_{l_{1}}<*^{\varepsilon}<t_{l_{1}+1} \leq \cdots \leq t_{l}
$$

where $u \leq v$ (resp. $u<v)$ means $v \in \tau^{[\varepsilon]}\left(\mathbb{R}_{\geq 0}\right) u\left(\right.$ resp. $v \in \tau^{[\varepsilon]}\left(\mathbb{R}_{>0}\right) u$ ). For a generic $*^{\varepsilon}$ (precisely, if $\omega_{W, 2}\left(*^{\varepsilon}\right) \neq 0$ ), the points $t_{1}, \ldots, t_{l}$ are distinct. Inside the complexification $\tau(\mathbb{C})\left(*^{\varepsilon}\right)$ of the line, we choose $l$-closed paths $\delta_{1}, \ldots, \delta_{l}$ based at $*^{\varepsilon}$ and turning once around the points $t_{1}, \ldots, t_{l}$ counter-clockwise as in the Fig. 6.

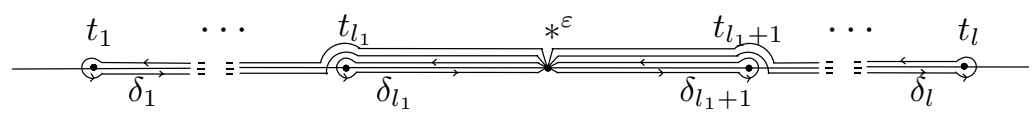

Figure 6. The Zariski-van Kampen generators on a $\tau$-orbit (cf. Fig.5.).

It is well-known that they generate the fundamental group of the complement of the discriminant locus and that their fundamental relations are determined by the Zariski-van Kampen method.

We compare the two generator systems introduced in $\S 4.1$ and in the present subsection. Let $a o^{\varepsilon}$ and $*^{\varepsilon}$ be the base points chosen in $\S 4.1$ and 
§4.3. The paths in $\mathcal{C}^{\{\varepsilon\}}$ connecting $a o^{\varepsilon}$ and $*^{\varepsilon}$ consist of a single homotopy class, denoted by $\left[a o^{\varepsilon}, *^{\varepsilon}\right]$, since $\mathcal{C}^{\{\varepsilon\}}$ is simply connected.

Theorem 4.2. The conjugation by $\left[a o^{\varepsilon}, *^{\varepsilon}\right]$ induces the bijection of the generator systems:

$$
\left\{\gamma_{\alpha}\right\}_{\alpha \in \Pi} \simeq\left\{\delta_{i}\right\}_{1 \leq i \leq l},
$$

where the bijection $\{1, \ldots, l\} \simeq \Pi$ of the index sets is given by the map $c_{W}$ : $i \leftrightarrow \alpha \Leftrightarrow c_{W}\left(t_{i}\right) \in H_{\alpha}$. The homotopy classes $\delta_{1}, \ldots, \delta_{l_{1}}$ mutually commute, and so do the homotopy classes $\delta_{l_{1}+1}, \ldots, \delta_{l}$.

\section{\$4.4. Proof of Theorem 4.2}

Let $*^{\varepsilon} \in \mathcal{C}^{\{\varepsilon\}}$ be the base point as above. Consider the real $\tau$-orbit $\tau^{[\varepsilon]}(\mathbb{R})\left(*^{\varepsilon}\right)$. Due to the homeomorphism (3.5.5), we identify the tube domain $\left(\pi_{\tau}^{[\varepsilon]}\right)^{-1}\left(E_{W}^{\{\varepsilon\}}\right)$ with $\left(\pi_{\Pi}\right)^{-1}\left(E_{\Gamma(W)}\right)$. For $\alpha \in \Pi_{1}$, the half line $\tau^{[\varepsilon]}\left(\sigma \varepsilon^{[h / 2]} \mathbb{R}_{>0}\right) \cdot *^{\varepsilon}$ intersects the hyperplane $H_{\alpha}=\left\{\phi_{\alpha}=0\right\}$ at a point, which we write $t_{\alpha}$. For $\alpha \in \Pi_{2}$, the other half line $\tau^{[\varepsilon]}\left(-\sigma \varepsilon^{[h / 2]} \mathbb{R}_{>0}\right) \cdot *^{\varepsilon}$ intersects the hyperplane $H_{\alpha}=\left\{\phi_{\alpha}=0\right\}$ at a point, which we write $t_{\alpha}$. If $\pi_{\tau}\left(*^{\varepsilon}\right) \notin B_{W, 2, \mathbb{R}}^{[\varepsilon]}$, then all $t_{\alpha}$ 's are distinct. We choose the paths as in Fig. 6. Let us index them by the set $\Pi$ : the path turning around the point $t_{\alpha}$ shall be called $\delta_{\alpha}$.

We first show that

i) the homotopy classes of $\delta_{\alpha}$ for $\alpha \in \Pi_{1}$ mutually commute and so do the homotopy classes of $\delta_{\alpha}$ for $\alpha \in \Pi_{2}$,

ii) for two choices of base points $*_{1}$ and $*_{2} \in \mathcal{C}^{\{\varepsilon\}}$, the conjugation by a path connecting $*_{1}$ and $*_{2}$ in $\mathcal{C}^{\{\varepsilon\}}$ induces one to one correspondences between the generators of the same index.

They follow from the descriptions of the discriminant locus and the central component in Theorem C, (5) and (6) as follows. Move the line $\tau^{[\varepsilon]}(\mathbb{R})(*)$ by moving $*$ in $\mathcal{C}^{\{\varepsilon\}}$ and trace the $l$-points $\left\{t_{\alpha}\right\}_{\alpha \in \Pi}=\tau^{[\varepsilon]}(\mathbb{R})(*) \cap D_{W, \mathbb{R}}^{[\varepsilon]}$ in the line. The fact that $E_{W}^{\{\varepsilon\}}$ does not intersect higher bifurcation set $B_{W, \geq 3}$ but only with ordinary $B_{W, 2, \mathbb{R}}^{\{\varepsilon\}}$ implies that one obtains only some commutative relations among generators. As far as $*$ moves inside $\mathcal{C}^{\{\varepsilon\}}$, the set of points $\left\{t_{\alpha} \mid \alpha \in \Pi_{1}\right\}$ and the set of the points $\left\{t_{\alpha} \mid \alpha \in \Pi_{2}\right\}$ are separated by the base point $*$ (Theorem $\mathrm{C}(6)$ ). Theorem $\mathrm{C}$ (5) claims that if $\alpha$ and $\beta$ belong to the same $\Pi_{1}$ or $\Pi_{2}$, then the hyperplanes $H_{\alpha}$ and $H_{\beta}$ are normal crossing in the tube domain $\left(\pi_{\tau, \mathbb{R}}^{[\varepsilon]}\right)^{-1}\left(\pi_{\tau, \mathbb{R}}^{[\varepsilon]}\left(\mathcal{C}^{\{\varepsilon\}}\right)\right) \subset\left(\pi_{\tau, \mathbb{R}}^{[\varepsilon]}\right)^{-1}\left(E_{W}^{\{\varepsilon\}}\right)$. This proves i) and ii).

Next, we show that

iii) the conjugation by a path connecting $a o^{\varepsilon}(\lambda)$ and $*^{\varepsilon}$ in $\mathcal{C}\{\varepsilon\}$ induces a correspondence of the homotopy class of $\gamma_{\alpha}$ to that of $\delta_{\alpha}$ for $\alpha \in \Pi$. 
We prove this by a use of ii) as follows.

For each $\alpha \in \Pi$, we can choose a base point $*_{\alpha}$ such that the line $\tau^{[\varepsilon]}(\mathbb{R})$. $*_{\alpha}$ and the discriminant locus $D_{W, \mathbb{R}}^{[\varepsilon]}$ intersect at the point $p_{\alpha}$ introduced in $\S 4.1$. Let $\left[a o^{\{\varepsilon\}}(\lambda), *_{\alpha}\right]$ be a path in $\mathcal{C}^{\{\varepsilon\}}$ connecting the two vertices and let $\left[*_{\alpha}, p_{\alpha}\right]$ be the interval in the line $\tau^{[\varepsilon]}(\mathbb{R}) \cdot *_{\alpha}$. Then, the path $\left[a o^{\{\varepsilon\}}(\lambda), p_{\alpha}\right]$ (the edge of $J_{W}^{\{\varepsilon\}}\left(\lambda^{[\varepsilon]}\right)$ connecting the vertices $a o^{\{\varepsilon\}}(\lambda)$ and $p_{\alpha}$ ) is homotopic to the composition of paths $\left[a o^{\{\varepsilon\}}(\lambda), *_{\alpha}\right]\left[*_{\alpha}, p_{\alpha}\right]$ in $\mathcal{C}^{\{\varepsilon\}}$. This means that the conjugation by $\left[a o^{\{\varepsilon\}}(\lambda), *_{\alpha}\right]$ induces the correspondence between the homotopy classes of $\gamma_{\alpha}$ and that of $\delta_{\alpha}$. This fact together with ii) implies iii).

Note. That the generator system $\left\{\delta_{\alpha}\right\}_{\alpha \in \Pi}$ satisfies the braid relations can be shown by the standard Zariski-van Kampen method.

\section{§4.5. Comparison of generator systems for $\varepsilon \in\{ \pm 1\}$}

Our identification of the fundamental group of $S_{W, \mathbb{C}} \backslash D_{W, \mathbb{C}}$ with the Artin group (either by the use of $\left\{\gamma_{\alpha}\right\}_{\alpha \in \Pi}$ or of $\left\{\delta_{\alpha}\right\}_{\alpha \in \Pi}$ ) depends on the choice of the base point locus. Actually, depending on $\varepsilon \in\{ \pm 1\}$, the base point is chosen in the central component $\mathcal{C}^{\{\varepsilon\}}$. For a path $\gamma$ connecting $\mathcal{C}^{\{+\}}$and $\mathcal{C}^{\{-\}}$, an isomorphism of the two fundamental groups is given by $\operatorname{Ad}_{[\gamma]}$, where $[\gamma]$ is the homotopy class of the path and by Ad we mean the conjugation action on homotopy classes.

Here we choose the simplest path connecting the two components $\mathcal{C}^{\{+1\}}$ and $\mathcal{C}^{\{-1\}}$. Namely, let the base points $a o^{+}$(resp. $a o^{-}$) lie on the positive (resp. negative) half v.o. axis $A O^{\varepsilon}$. Consider the complexification of the v.o. axis. Inside the complex v.o. axis deleted by the origin, let $\gamma_{+}\left(\right.$resp. $\left.\gamma_{-}\right)$be the path connecting $a o^{+}$to $a o^{-}$(resp. $a o^{-}$to $a o^{+}$) by turning half around the origin counter-clockwise.

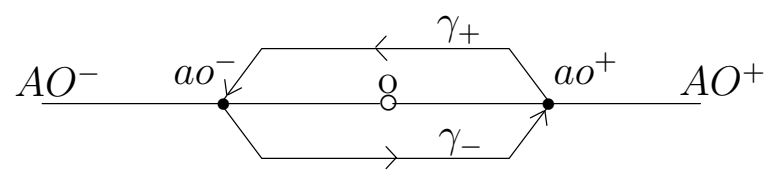

Figure 7. The complexification of the vertex orbit axis $A O$

By the use of them, we have the isomorphisms:

$$
\begin{aligned}
& \pi_{1}\left(S_{W, \mathbb{C}} \backslash D_{W, \mathbb{C}}, a o^{+}\right) \stackrel{\operatorname{Ad}_{\left[\gamma_{+}\right]}}{\longleftrightarrow} \pi_{1}\left(S_{W, \mathbb{C}} \backslash D_{W, \mathbb{C}}, a o^{-}\right) \\
& \pi_{1}\left(S_{W, \mathbb{C}} \backslash D_{W, \mathbb{C}}, a o^{+}\right) \stackrel{\operatorname{Ad}_{\left[\gamma_{-}\right]}}{\longleftarrow} \pi_{1}\left(S_{W, \mathbb{C}} \backslash D_{W, \mathbb{C}}, a o^{-}\right)
\end{aligned}
$$


In order to state the following Assertion, we recall the fundamental element [BS]. Consider the monoid generated by the letters $a_{\alpha}(\alpha \in \Pi)$ satisfying the braid relations as the defining relations. The fundamental element $\Delta$ is the shortest element in the monoid which is divisible (from both sides) by any of the generators $a_{\alpha}$. Such $\Delta$ exists uniquely in the monoid. Since the monoid is embedded in the Artin group, we identify $\Delta$ with its image.

Assertion 4.3. The homotopy classes $\left[\gamma_{+}\right]\left[\gamma_{-}\right]$and $\left[\gamma_{-}\right]\left[\gamma_{+}\right]$are the fundamental element $\Delta$ in each of the fundamental group based at ao ${ }^{+}$and $a o^{-}$regarded as an Artin group with respect to the generator systems $\left\{\gamma_{\alpha}\right\}_{\alpha \in \Pi}$.

This fact follows from i) the length of $\left[\gamma_{+}\right]\left[\gamma_{-}\right]$as an element of the monoid is given by $l\left(\left[\gamma_{+}\right]\left[\gamma_{-}\right]\right)=\operatorname{deg}\left(\Delta_{W}\right) / \operatorname{deg}(R)=h l / 2=l(\Delta)$, and ii) a description of the monodromy of $\left[\gamma_{+}\right]\left[\gamma_{-}\right]$on $b_{W}: T_{W, \mathbb{C}}-\rightarrow V_{\Pi, \mathbb{C}}$, see $\left.[\mathrm{S} 4, \S 8,9]\right)$.

Note. $\quad 1 . \Delta^{2}$ belongs to the center of the Artin group for any type of $W$. However, $\Delta$ does not belong to the center if $W$ is of type $A_{n}$ for $n \geq 2, D_{2 k+1}$ for $k \geq 2, E_{6}$ and $I_{2}(2 q+1)$ for $q \geq 1$.

2. The fundamental element $\Delta$ projects to the longest element of the Coxeter group $W$.

\section{Appendix. Dihedral Group of Type $I_{2}(h)$}

For the dihedral group $W\left(I_{2}(h)\right)(h \geq 3)$, we describe the data $1-9$.

1. The action of $W\left(I_{2}(h)\right)$ on $U$ (recall Notation $\left.\left.\left.\S 2.5 \mathrm{i}\right)-\mathrm{vi}\right)\right)$ :

$W\left(I_{2}(h)\right)$ is generated by the reflections $\alpha_{1}$ and $\alpha_{2}$ on $U:=\mathbb{R} \oplus \mathbb{R} i$ given by $\alpha_{1}(z):=\bar{z}$ and $\alpha_{2}(z):=\omega^{2} \bar{z}$, where $z:=x+y i \in U$ and $\omega:=\exp (\pi i / h)$, which satisfy the fundamental relation $\left(\alpha_{1} \alpha_{2}\right)^{h}=1$.

2. The set of reflections of $W\left(I_{2}(h)\right)$ is given by

$$
R\left(W\left(I_{2}(h)\right)\right)=\left\{\alpha_{k}:=\alpha_{1}\left(\alpha_{1} \alpha_{2}\right)^{k-1} \mid k=1, \ldots, h\right\} .
$$

3. The normalizer $N\left(W\left(I_{2}(h)\right)\right)$ in $\mathrm{GL}(U)$ is equal to $W\left(I_{2}(2 h)\right)$ and $N\left(W\left(I_{2}(h)\right)\right) / W\left(I_{2}(h)\right)=\{[1],[\beta]\}$ where $\beta$ is the reflection: $\beta(z)=\omega \bar{z}$. One has $[-1]=[1]$ for even $h$, and $[-1]=[\beta]$ for odd $h$.

4. The twisted real vertex orbit planes are given by

$$
S_{W\left(I_{2}(h)\right), \mathbb{R}}^{[+1]}=\operatorname{Spec}(\mathbb{R}[R, S])_{\mathbb{R}} \text { and } S_{W\left(I_{2}(h)\right), \mathbb{R}}^{[\beta]}=\operatorname{Spec}\left(\mathbb{R}\left[R, S^{[\beta]}\right]\right)_{\mathbb{R}},
$$

where $R=R^{[\beta]}=x^{2}+y^{2}, S=S^{[1]}=\operatorname{Re}\left((x+i y)^{h}\right)$ and $S^{[\beta]}=S / \sqrt{-1}$. 
5. For $[u] \in N(W) / W$, the twisted real discriminant locus $D_{W\left(I_{2}(h)\right), \mathbb{R}}^{[u]}$ in $S_{W\left(I_{2}(h)\right), \mathbb{R}}^{[+1]}$ is defined by the equation:

$$
\Delta_{W\left(I_{2}(h)\right)}=R^{h}-S^{2}=R^{h}+\left(S^{[\beta]}\right)^{2}=\varepsilon^{h}\left((\varepsilon R)^{h}-\left(S^{[\varepsilon]}\right)^{2}\right)=0 .
$$

This implies that $D_{W\left(I_{2}(h)\right), \mathbb{R}}^{[\beta]}=\{0\}$ for even $h$, but $D_{W\left(I_{2}(h)\right), \mathbb{R}}^{[\varepsilon]} \neq\{0\}$ for any $\varepsilon$. Therefore, in 7. and 8., we consider only cases for $[\varepsilon]$.

6. The $\tau^{[u]}$-action on the plane: $\left(R, S^{[u]}\right) \mapsto\left(R, S^{[u]}+\lambda^{[u]}\right)$ for $\lambda^{[u]} \in \mathbb{R}$,

7. The equation for the inverse image $\left(\pi_{W, \mathbb{R}}^{[\varepsilon]}\right)^{-1}\left(D_{W\left(I_{2}(h)\right), \mathbb{R}}^{[\varepsilon]}+\lambda^{[\varepsilon]}\right)$ by the polar coordinates $x+i y=\sqrt{\varepsilon} \operatorname{rexp}(i \theta)$ on $U^{\varepsilon}$ is given by

$$
\begin{aligned}
& \left(\tau^{[\varepsilon]}\right)^{*}\left(\lambda^{[\varepsilon]}\right) \Delta_{W}=R^{h}-\varepsilon^{h}\left(S^{[\varepsilon]}+\lambda^{[\varepsilon]}\right)^{2} \\
= & \varepsilon^{h}\left(r^{2 h} \sin ^{2}(h \theta)-2 \varepsilon^{h(h-1) / 2} r^{h} \lambda^{[\varepsilon]} \cos (h \theta)-\left(\lambda^{[\varepsilon]}\right)^{2}\right) \\
= & \varepsilon^{h}\left(r^{h}\left(1-\varepsilon^{h(h-1) / 2} \cos (h \theta)\right)-\lambda^{[\varepsilon]}\right)\left(r^{h}\left(1+\varepsilon^{h(h-1) / 2} \cos (h \theta)\right)+\lambda^{[\varepsilon]}\right)
\end{aligned}
$$

8. The dual polyhedron is described by the polar coordinates as

$$
\begin{aligned}
\bar{K}_{W}^{\varepsilon}\left(\lambda^{[\varepsilon]}\right)= & \left\{z \in U^{\varepsilon} \mid \tau^{[\varepsilon]}\left(\lambda^{[\varepsilon]}\right) \Delta(z) \leq 0\right\} \cap\left\{\tau^{[\varepsilon]}\left(-\lambda^{[\varepsilon]}\right) \Delta(z) \leq 0\right\} \\
= & \left\{\sqrt{\varepsilon} r \exp (i \theta) \in U^{\varepsilon} \mid r^{h} \leq \lambda^{[\varepsilon]} /\left(1-\varepsilon^{h(h-1) / 2} \cos (h \theta)\right)\right. \\
& \left.r^{h} \leq \lambda^{[\varepsilon]} /\left(1+\varepsilon^{h(h-1) / 2} \cos (h \theta)\right)\right\} .
\end{aligned}
$$

9. For $h=3$ and 4 , for $\varepsilon=1$ or -1 and for $\lambda^{[\varepsilon]}=1$, we draw the figures:

i) the real discriminant locus $D_{W, \mathbb{R}}^{[\varepsilon]}$ and the $\lambda^{[\varepsilon]}$-shifted real discriminant locus: $D_{W, \mathbb{R}}^{[\varepsilon]} \pm \lambda^{[\varepsilon]}:=\tau^{[\varepsilon]}\left( \pm \lambda^{[\varepsilon]}\right)\left(D_{W, \mathbb{R}}^{[u]}\right)$ in $S_{W\left(I_{2}(h)\right), \mathbb{R}^{*}}^{[\varepsilon]}$

ii) the inverse images $\left(\pi_{W, \mathbb{R}}^{[\varepsilon]}\right)^{-1}\left(D_{W, \mathbb{R}}^{[\varepsilon]}\right)$ and $\left(\pi_{W, \mathbb{R}}^{[\varepsilon]}\right)^{-1}\left(D_{W, \mathbb{R}}^{[\varepsilon]} \pm \lambda^{[\varepsilon]}\right)$ in $U^{\varepsilon}$.

iii) the parallelograms $J_{W}^{\{\varepsilon\}}\left(\lambda^{[\varepsilon]}\right)$ and the polyhedra $K_{W}^{\varepsilon}\left(\lambda^{[\varepsilon]}\right)$ (shaded).

iv) the two twisted real forms $S_{W\left(I_{2}(h)\right), \mathbb{R}}^{[1]}$ and $S_{W\left(I_{2}(h)\right), \mathbb{R}}^{[\beta]}$ which are embedded in the real 3-space $S_{W\left(I_{2}(h)\right), \mathbb{C}} \cap\{\operatorname{Im}(R)=0\}$.

Remark 14. We compare the multiplicities of two equations of the bifurcation set in the vertex orbit curve $T_{W\left(I_{2}(h)\right)}:=\operatorname{Spec}(\mathbb{R}[R])$.

i) The discriminant $\delta$ of the map $R=z \bar{z}=x^{2}+y^{2}: U \rightarrow \mathbb{R}$ is equal to $R$ itself. (The free resolution of $\mathbb{R}[x, y] /\left(\partial_{x} R, \partial_{y} R\right)$ as an $\mathbb{R}[R]$-module is given by $0 \rightarrow \mathbb{R}[R] \stackrel{R}{\rightarrow} \mathbb{R}[R] \rightarrow \mathbb{R}[x, y] /\left(\partial_{x} R, \partial_{y} R\right) \rightarrow 0$. $)$

ii) The discriminant $\omega$ of the quadratic polynomial $\Delta_{W\left(I_{2}(h)\right)}=R^{h}-S^{2}$ in $S$ is equal to $4 \cdot R^{h}$.

Comparing i) and ii), we obtain a relation:

$$
\omega=4 \cdot \delta^{h} \text {. }
$$


1. $\mathbf{h}$ is odd and coset $[1]$ : In this case, $[\beta]=[-1] \notin\left[W\left(I_{2}(h)\right)\right]$.

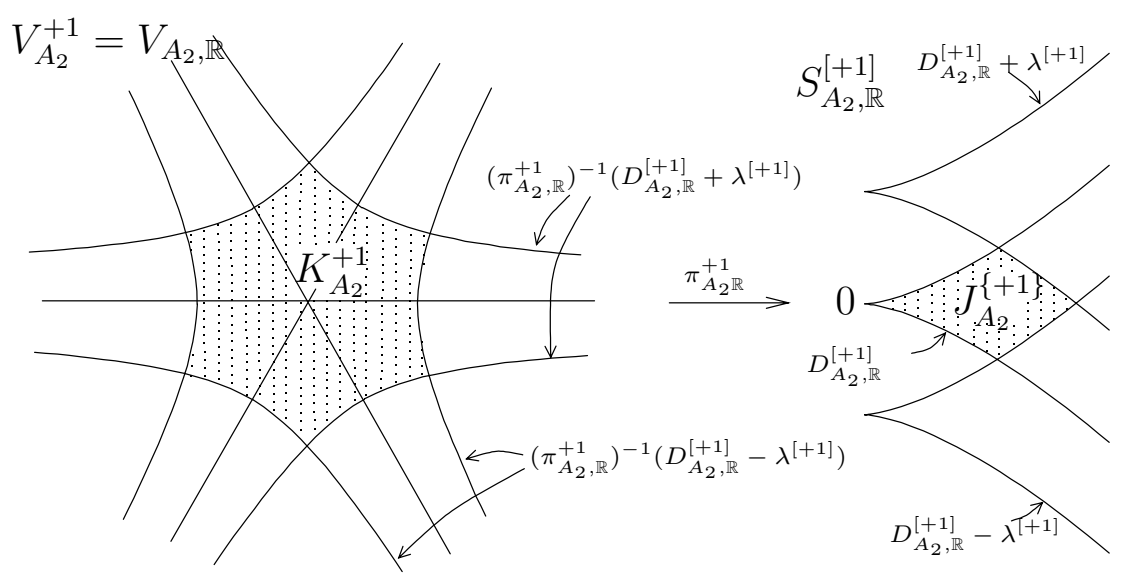

3 lines in $V_{A_{2}}^{+}$are the reflection hyperplanes for $W_{A_{2}}$.

Figure 8. Polyhedra $J_{A_{2}}^{\{+1\}}$ and $K_{A_{2}}^{+1}$ for $\lambda^{[+1]}=1$

2. $\mathbf{h}$ is odd and coset $[\beta]=[-1]$ :

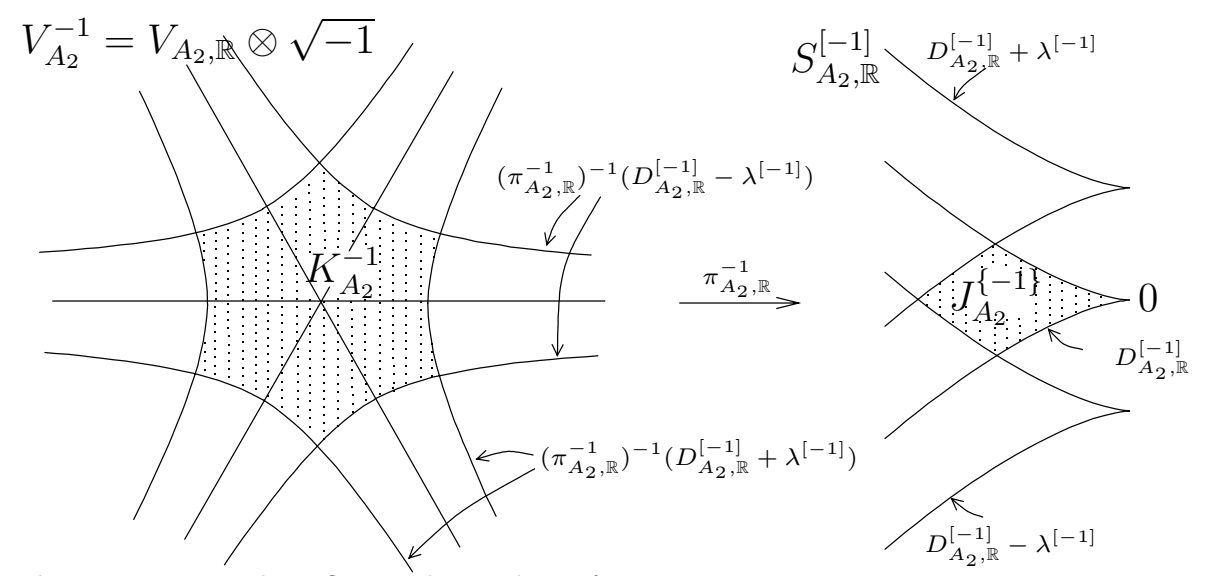

3 lines in $V_{A_{2}}^{-}$are the reflection hyperplanes for $W_{A_{2}}$.

Figure 9. Polyhedra $J_{A_{2}}^{\{-1\}}$ and $K_{A_{2}}^{-1}$ for $\lambda^{[-1]}=1$ 


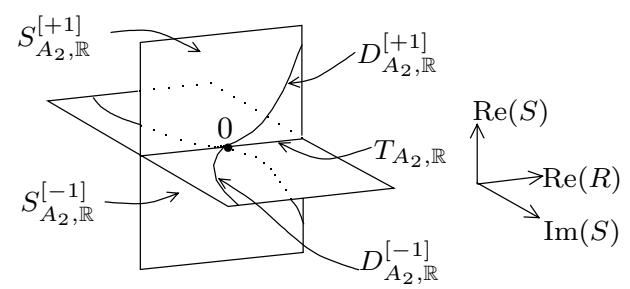

Figure 10. Positions of $S_{A_{2}, \mathbb{R}}^{[+1]}$ and $S_{A_{2}, \mathbb{R}}^{[-1]}$ inside $S_{A_{2}, \mathbb{C}} \cap\{\operatorname{Im}(R)=0\}$.

3. $\mathbf{h}$ is even and coset $[1]=[-1]$ : In this case, $-1 \in W\left(I_{2}(h)\right)$.

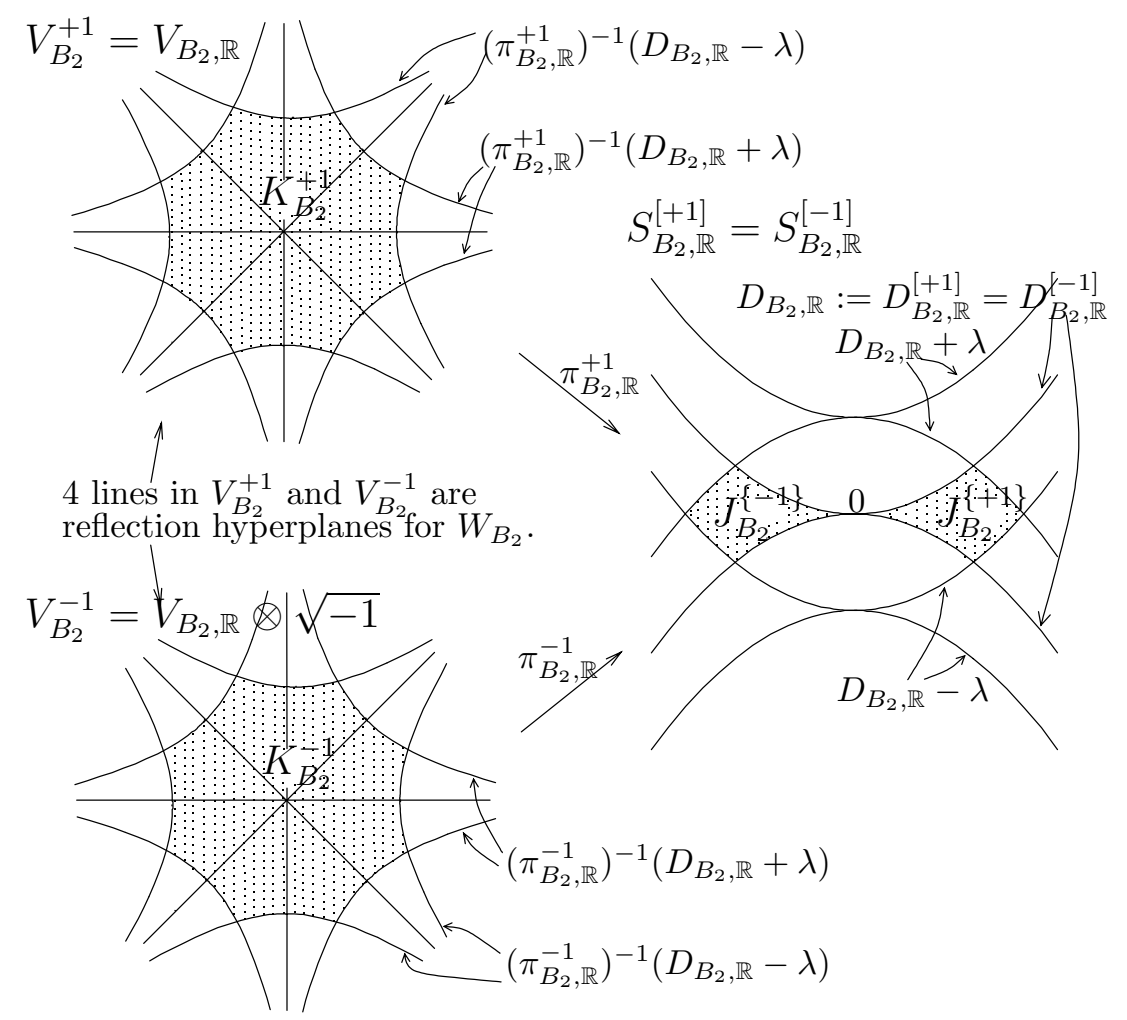

Figure 11. Polyhedra $J_{B_{2}}^{\{ \pm 1\}}(\lambda)$ and $K_{B_{2}}^{ \pm 1}(\lambda)$ for $\lambda=1$. 
4. $\mathbf{h}$ is even and coset $[\beta]$ : In this case, $[\beta] \neq[-1] \in\left[W\left(I_{2}(h)\right)\right]$. The discriminant locus $D_{W\left(I_{2}(h)\right), \mathbb{R}}^{[\beta]}$ in the real form $S_{W\left(I_{2}(h)\right), \mathbb{R}}^{[\beta]}$ consists only of the origin $\{o\}$. Therefore, we omit the figure for this case.

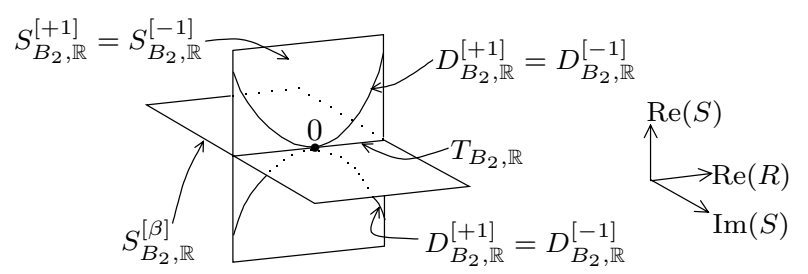

Figure 12. Positions of $S_{B_{2}, \mathbb{R}}^{[ \pm 1]}$ and $S_{B_{2}, \mathbb{R}}^{[\beta]}$ inside $S_{B_{2}, \mathbb{C}} \cap\{\operatorname{Im}(R)=0\}$.

\section{References}

[Ar1] Arnol'd, Vlasimir, I., Springer numbers and morsification spaces, J. Alg. Geom., 1 (1992), 197-214.

[Ar2] The calculus of snakes and combinatorics of Bernoulli, Euler and Springer numbers of Coxeter groups, Russian Math. Surveys, 47 (1992), 1-51.

[A] Artin, Emil, Theorie der Zöpfe, Abhandlungen aus dem Mathematischen Seminar der Hamburgishen Universität, 4 (1926), 47-72.

[B] Bourbaki, N., Elements de mathematique, Fasc. XXXIV, Groupes et algebres de Lie, Chs. 4-6, Hermann, Paris 1968.

[Br1] Brieskorn, Egbert, Die Fundamental Gruppe des Raumes der Regulären Orbits einer endlichen komplexen Spiegelungsgruppe, Invent. Math., 12 (1971), 57-61.

[Br2] Sur les groupes de tresses. Sém. Bourbaki, (1971).

[BS] Brieskorn, Egbert and Saito, Kyoji, Artin Gruppen und Coxeter Gruppen, Invent. Math., 17 (1972), 245-271.

[Ch] Chevalley, Claude, Invariants of finite groups generated by reflections, Amer. J. Math., 77 (1955), 778-782.

[C] Coleman, A. John: The Betti numbers of the simple Lie groups, Canad. J. Math., 10 (1958), 349-356.

[D1] Deligne, Pierre, Les immeubles des groupes de tresses généralisé, Invent. Math., 17 (1972), 273-302.

[D2] $\longleftarrow$, Action du groupe des tresses surs categorie, Invent. Math., 128 (1997) 159175.

[Hi] Hilbert, David, Under die Theorie der algebraischen Formen, Math. Ann., 36 (1890), 473-534.

[Hu] Hung, Nguyen H.V., The modulo 2 cohomology algebras of symmetric groups, Japan. J. Math., 13 (1987), 169-208.

[K] Kostant, Bernard, The principal three-dimensional subgroup and the Betti numbers of a complex simple group, Amer. J. Math., 81 (1959), 973-1032.

[Lo1] Looijenga, Eduard, The complement of the bifucation variety of a simple singularity, Invent. Math., 23 (1974), 105-116.

[Lo2] $\ldots$, The Discriminant of a real simple singularity, Compositio Math., 37 (1978), 51-62.

[Ly] Lyashko, O.V., The geometry of the bifucation diagrams, Usp. Math. Nauk, 34 (1979), 205-206; Russian Math. Surveys, 34 (1979), 209-210. 
[M] Huynh Mui, Duality on the infinite symmetric products, Acta Math. Vietnam, 5 (1980), 100-149.

[N] Nakamura, Tokushi, On cohomology operations, Japan. J. Math., 33 (1963), 93-145.

[P-S] Procesi, Claudio and Schwarz, Gerald, Inequalities defining orbit spaces, Invent. Math., 81 (1985), 539-554.

[S1] Saito, Kyoji, On a linear structure of the quotient variety by a finite reflexion group, Publ. RIMS, Kyoto Univ., 29 (1993), 535-579.

[S2] _ Period mapping associated to a primitive form, Publ. RIMS, Kyoto Univ., 19 (1983), 1231-1264.

[S3] Uniformization of the orbifold of a finite reflection group, in Frobenius Manifolds, C. Hertling, M. Marcolli (eds.), Aspect Math., E36 (2004), 265-320.

[S4] Polyhedra dual to the Weyl chamber decomposition, in preparation.

[Sa] Salvetti, Mario, Topology of the complement of real hyperplanes in $\mathbb{C}^{n}$, Invent. Math., 83 (1987), 603-618.

[So] Solomon, Louis: A decomposition of the group algebra of a finite Coxeter group, $J$. Alg., 9 (1968), 220-239.

[Sp1] Springer, Tony A., Remarks on a combinatorial problem, Nieuw Arch. Wisk. (1), 19 (1971), 30-36.

[Sp2] , Reductive Groups, Proc. Sympos. Pure Math., 33 (1979), Part 1, 3-27.

[Te] Terao, Hiroaki, The Bifucation set and logarithmic vector fields, Math. Ann, 263 (1983), 313-321.

[T1] Thom, René, L'équivalence d'une fonction différentiable et d'un polynome, Topology, 3 (1965), 297-307.

[T2] _ Stabilité Structurelle et Morphogénèse, W.A. Benjamin, Inc., Advanced Book Program, Reading, Massachusetts 01867 